\title{
Solar Wind Interaction and Impact on the Venus Atmosphere
}

\author{
Yoshifumi Futaana $^{1}$. Gabriella Stenberg Wieser ${ }^{1}$. \\ Stas Barabash ${ }^{1}$. Janet G. Luhmann²
}

Received: 6 December 2016 / Accepted: 30 March 2017 / Published online: 16 August 2017

(C) The Author(s) 2017. This article is published with open access at Springerlink.com

\begin{abstract}
Venus has intrigued planetary scientists for decades because of its huge contrasts to Earth, in spite of its nickname of "Earth's Twin". Its invisible upper atmosphere and space environment are also part of the larger story of Venus and its evolution. In 60s to 70s, several missions (Venera and Mariner series) explored Venus-solar wind interaction regions. They identified the basic structure of the near-Venus space environment, for example, existence of the bow shock, magnetotail, ionosphere, as well as the lack of the intrinsic magnetic field. A huge leap in knowledge about the solar wind interaction with Venus was made possible by the 14-year long mission, Pioneer Venus Orbiter (PVO), launched in 1978. More recently, ESA's probe, Venus Express (VEX), was inserted into orbit in 2006, operated for 8 years. Owing to its different orbit from that of PVO, VEX made unique measurements in the polar and terminator regions, and probed the near-Venus tail for the first time. The near-tail hosts dynamic processes that lead to plasma energization. These processes in turn lead to the loss of ionospheric ions to space, slowly eroding the Venusian atmosphere. VEX carried an ion spectrometer with a moderate mass-separation capability and the observed ratio of the escaping hydrogen and oxygen ions in the wake indicates the stoichiometric loss of water from Venus. The structure and dynamics of the induced magnetosphere depends on the prevailing solar wind conditions. VEX studied the response of the magnetospheric system on different time scales. A plethora of waves was identified by the magnetometer on VEX; some of them were not previously observed by PVO. Proton cyclotron waves were seen far upstream of the bow shock, mirror mode waves were observed in magnetosheath and whistler mode waves, possibly generated by lightning discharges were frequently seen. VEX also encouraged renewed numerical modeling efforts, including fluid-type of models and particle-fluid hybrid type of models, describing the plasma interaction on scales ranging from ion gyro radius to the entire induced magnetosphere. In this review article, we review what has been
\end{abstract}

Venus III

Edited by Bruno Bézard, Christopher T. Russell, Takehiko Satoh, Suzanne E. Smrekar and Colin F. Wilson

\begin{tabular}{ll}
\hline$凶$ & Y. Futaana \\
futaana@irf.se \\
1 & Swedish Institute of Space Physics, Kiruna, Sweden \\
2 & University of California, Berkeley, Space Sciences Laboratory, Berkeley, CA, USA
\end{tabular}


found from space physics measurements around Venus (from the solar wind down to the ionopause), with a particular emphasis on updated results since the Venus Express mission. We conclude the article by a short discussion on the remaining open scientific questions and the future of this field.

Keywords Venus · Solar wind · Plasma · Venus express · Erosion · Atmospheric loss · Proton cyclotron wave $\cdot$ Induced magnetosphere

\section{Introduction: How Space Physics Fits into the Overall Exploration of Venus}

Interest in Venus is largely due to its dramatic differences from Earth in spite of its similar size and position in the solar system. Its history must clearly have involved events and processes that were (and in some cases still are) distinctively different, or a set of probabilistic outcomes during its formation and evolution that contrasted sharply with those that occurred on and within our home planet. One major early finding from the Soviet Venus missions and the US Mariner missions - besides the existence of the dense, cloudy, and caustic atmospheric greenhouse-was the apparent lack of an Earth-like space environment. Our planet is enveloped deep inside a space extending to at least $\sim 10$ Earth radii toward the Sun direction carved out in the interplanetary medium by its intrinsic large scale dipolar magnetic field, influencing more than 3000 Earth radii in the anti-Sunward direction (e.g. Vaisberg et al. 1972; Intriligator et al. 1979). Spacecraft exploring Earth-space thus found many features such as radiation belts inside this space, with the solar wind plasma from the Sun gaining direct access to Earth's atmosphere only in limited regions at polar latitudes. As reviewed in this paper, Venus's atmosphere is instead directly and globally exposed to the solar wind's influence, with consequences that produce a host of different, and some analogous, physical phenomena. A major question is what role this property has played in determining what we see at Venus today. In particular, the direct solar wind interaction represents one way that energy has been transferred from the Sun to the planet's atmosphere over time, possibly altering its structure, composition and chemistry. Venus may once have had an ocean (see the accompanying review article by Gilmore et al. 2017) whose constituents were either lost to space (as suggested by the measured large D/H ratio there), or taken up by the surface (e.g. by oxidation). Solar wind interaction processes, combined with Venus' atmospheric photochemistry, turn out to be an effective way to accelerate some of the heavier constituents from its $\mathrm{CO}_{2}$ dominated atmosphere to escape velocities. By investigating what is happening today we can constrain the possible long term impacts of the solar wind interaction in order to resolve the ongoing debate concerning planetary magnetic fields as 'shields', protecting planetary atmospheres from Venus-like fates. We also learn more about the very different ways in which planetary bodies can interact with their central stars, providing perspective on both other weakly magnetized solar system bodies (including planetary satellites) and exoplanet-stellar host pairings.

In 1960s to 70s, several missions explored the Venus-solar wind interaction region. Perturbations related to the bow shock were measured by Venera-4, -6, - 9 and -10 as well as Mariner-5 and -10 . Venera-9 and -10 passed through the magnetotail, which was found to be controlled by the upstream IMF direction (e.g. Vaisberg et al. 1976). The detection of the low-energy ions (100-200 eV) in the magnetotail was also reported (Vaisberg et al. 1976).

As of the early 1990s the main regions and boundaries of induced magnetosphere of Venus had been extensively studied with Pioneer Venus Orbiter (PVO) (e.g. Russell and 


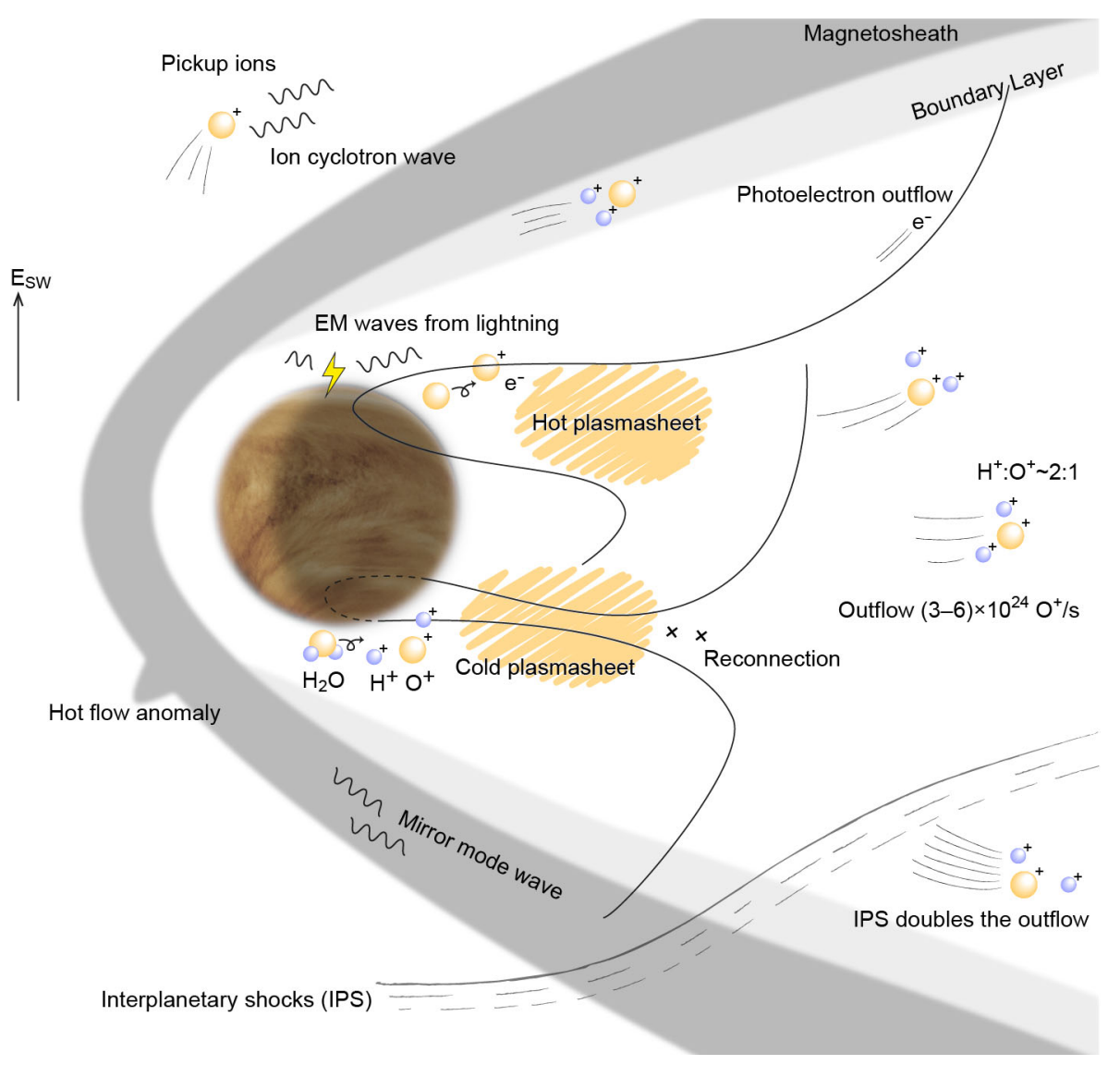

Fig. 1 An illustration summarizing the Venus Express findings on the space environment of Venus

Vaisberg 1983). At solar minimum, however, the altitude of PVO was too high to observe the near-Venus environment. As with all missions, the PVO data also had other orbital biases and in particular left in-situ observational gaps at both high latitudes and in the solar wind wake. And although state-of-the-art at the time, the magnetometer and plasma instruments on PVO had low temporal, energy and angular resolution compared to more recent capabilities, and the energetic plasma analyzer had no mass discrimination.

The Venus Express (VEX) measurements have therefore significantly complemented and expanded upon previous observations. While overall, they confirmed PVO results, the new instrumentation revealed more details and processes relevant to the solar wind interaction and its effects than were previously known. Here we briefly summarize what VEX observed during its 9 years in orbit. For context, it is important to remember that the solar activity level during the VEX mission was low to moderate compared to the period of the primary PVO mission, and that consequences of that, including lower solar EUV fluxes and weaker solar wind (Hathaway 2015; McComas et al. 2008; Emmert et al. 2010) must be considered in viewing the Venus plasma interaction results as a whole.

In this review article, we focus on summarizing VEX findings and updates building on the previous Venera, Mariner and PVO results. Figure 1 illustrate selected findings emphasizing in this article. These findings add to the excellent outcomes from those previous observa- 
tions. We refer readers interested in further background to several articles (e.g. Bridge et al. 1976; Breus 1979; Hunten et al. 1983; Luhmann 1986; Russell 1991; Luhmann et al. 1992; Bougher et al. 1997) providing more details from various perspectives.

\section{A Brief Overview of Space Physics Exploration at Venus}

Venus has been explored for more than 55 years. The first attempts at Venus exploration were the twin Soviet 1VA probes (Sputnik-7 and Venera-1), launched on February 4 and 12, 1961. They unfortunately failed to reach their destination. Mariner-2, launched on August 27, 1962 , successfully flew by Venus at the altitude of $35,000 \mathrm{~km}$. In fact, this is the first successful planetary flyby. In 1967, Venera-4 probed the Venusian atmosphere down to $25 \mathrm{~km}$. One day after, Mariner-5 flew-by Venus, allowing the inter-comparison of the measurements. The upper limit of the Venusian intrinsic magnetic field was inferred to be $10^{-5}$ times than that of the Earth (Dolginov et al. 1969). Bow shock and its related phenomena were also detected (Gringauz et al. 1968; Dolginov et al. 1968). These persistent features were measured by the subsequent Mariner-10 mission (Bridge et al. 1976). The first space probe to land on the surface of Venus was Venera-7 in 1970. Venera-7 was the first probe to send the data from an extra-terrestrial planetary surface. However, due to the high temperature at the Venusian surface, Venera-7 survived only 23 min. No plasma related instruments were included in its payload.

A milestone in terms of the space physics around Venus-the Venus-solar wind interaction-was achieved by Venera- 9 and -10 . They orbited the near-Venus space environment through the nightside tail region where they detected the bow shock, induced magnetosphere boundary, and magnetotail (e.g. Vaisberg et al. 1976; Gringauz et al. 1976). Of particular importance was the measurement of low-energy (100-200 eV) ions in the induced magnetosphere boundary, which escaped into space through the magnetotail (Vaisberg et al. 1976).

The active era of the Venus exploration continued until mid 90s and finished with the loss of Magellan during aerobraking in 1993. However, the last spacecraft during this period equipped with instruments relevant for the solar wind interaction studies was Pioneer Venus Orbiter (PVO), which was launched in 1978 and operational until 1992. The era of intense Venus investigations was followed by a 13-year pause, which was concluded with the launch of Venus Express (VEX) in 2005. After more than 8 years of operation, VEX used up all its fuel, and went down into the atmosphere. Currently (in 2017), the only Venus spacecraft still alive is Akatsuki, although it does not carry instrumentation for space physics.

The exploration of Venus by spacecraft has been an important addition to the ground based observations in the efforts to understand our sister planet in detail. Many of the spacecraft missions have carried instruments to investigate the plasma environment of the planet in order to understand Venus interaction with the solar wind. Table 1 summarizes the missions to Venus and the instrumentation relevant for studying plasma processes around Venus.

The range in energy coverage of the particle instruments is shown in Fig. 2. It can be seen that many missions have carried out plasma environment investigation under various phases of the solar cycle, which is one of the main drivers of the changes in the Venussolar wind interaction. The plasma investigations made by the early missions led to a large interest in further understanding the planet's interaction with the solar wind. These missions introduced many of the scientific questions that later became the focus for the long Pioneer Venus program. 


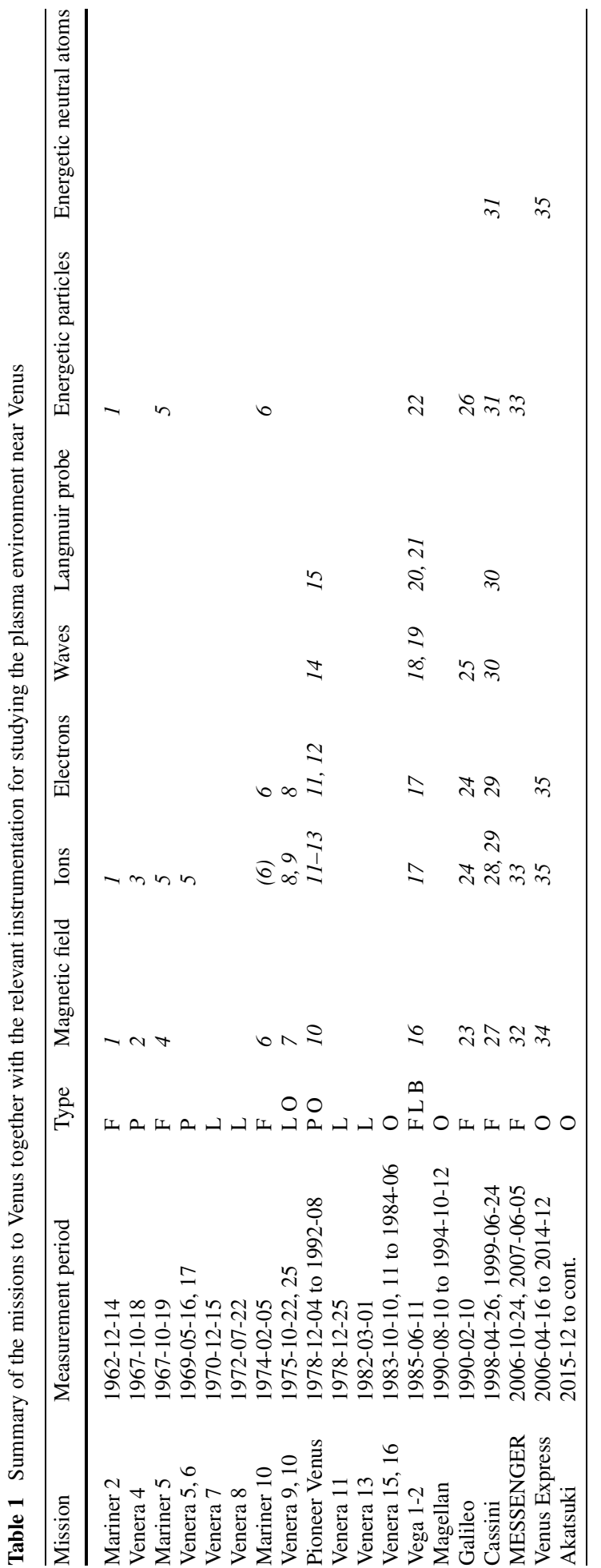

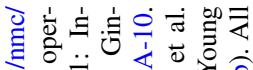

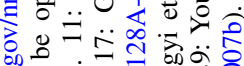

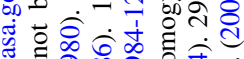

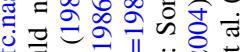

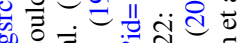

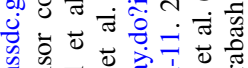

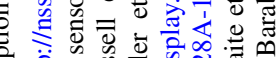

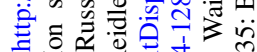

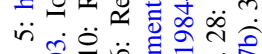

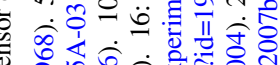

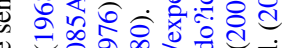
छ

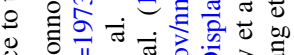
రั 过宁

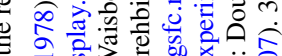
च.

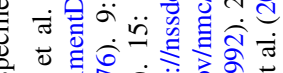

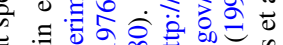

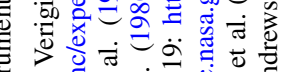

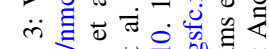

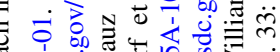

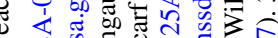

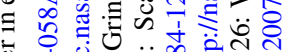

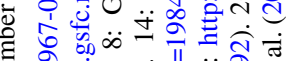

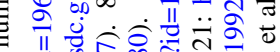

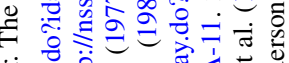

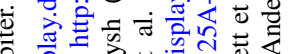

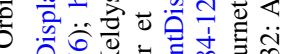
○ ส ธี ญั

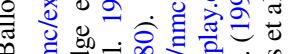

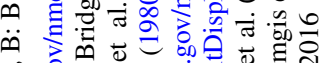
过 我

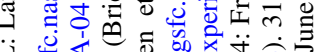
讨茄 \& ڤ.

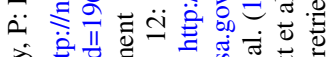

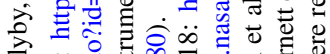
新

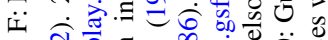

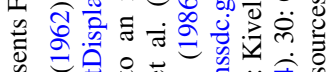

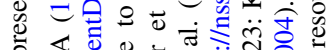

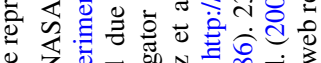

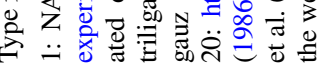



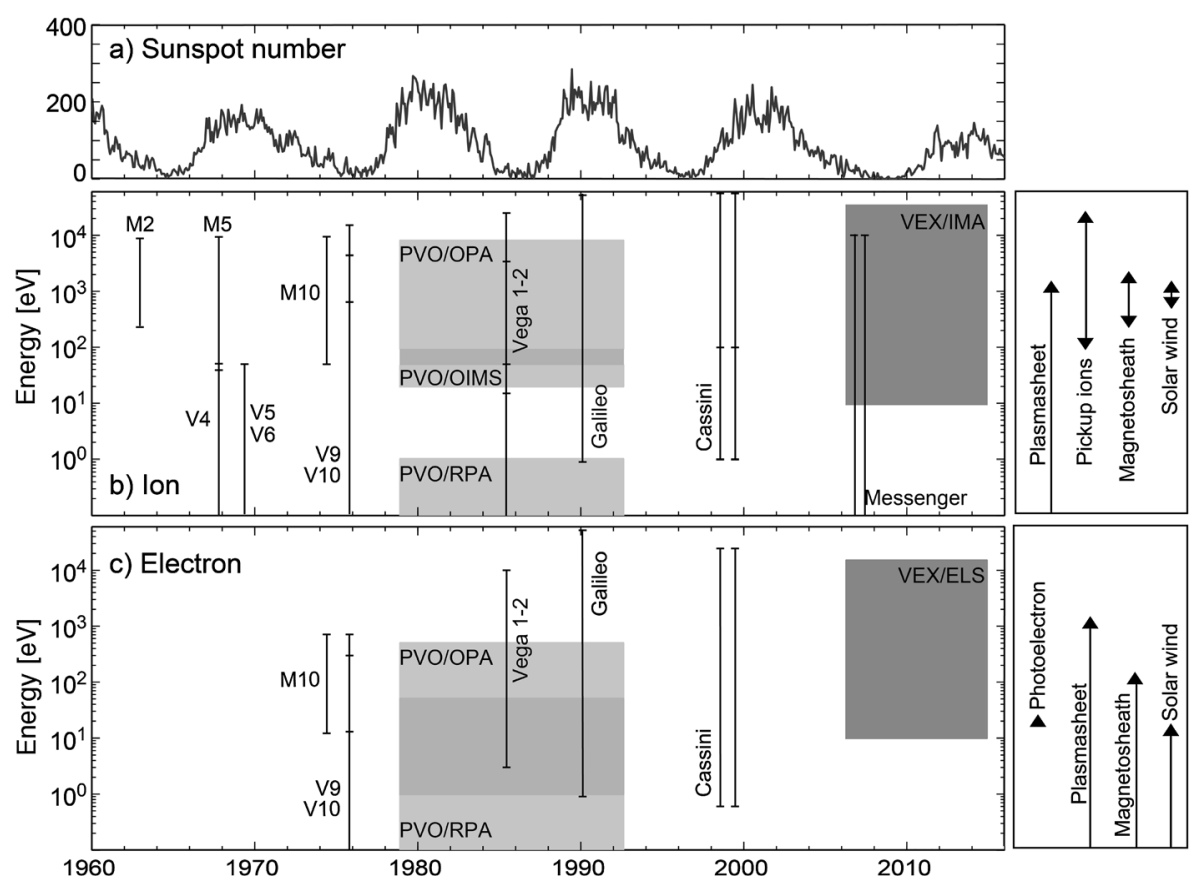

Fig. 2 (a) Sunspot number, representing the activity of the Sun (the sunspot number data is provided by WDC-SILSO, Royal Observatory of Belgium, Brussels) (SILSO 1960-2015). (b) Ion and (c) electron sensors carried by missions to Venus, both their energy ranges and exploration time are shown. "M" refers to the Mariner series, and "V" to the Venera series. In the boxes to the right of the panels, typical energy ranges for different plasma regions in the Venus environment are indicated

Fig. 3 Typical orbits of the Pioneer Venus Orbit (PVO) and Venus Express (VEX) spacecraft. VEX accessed different regions in the Venusian magnetosphere compared to PVO, which enabled new discoveries and complementary observations. After the aerobraking in mid 2014, VEX apocenter altitude was reduced slightly

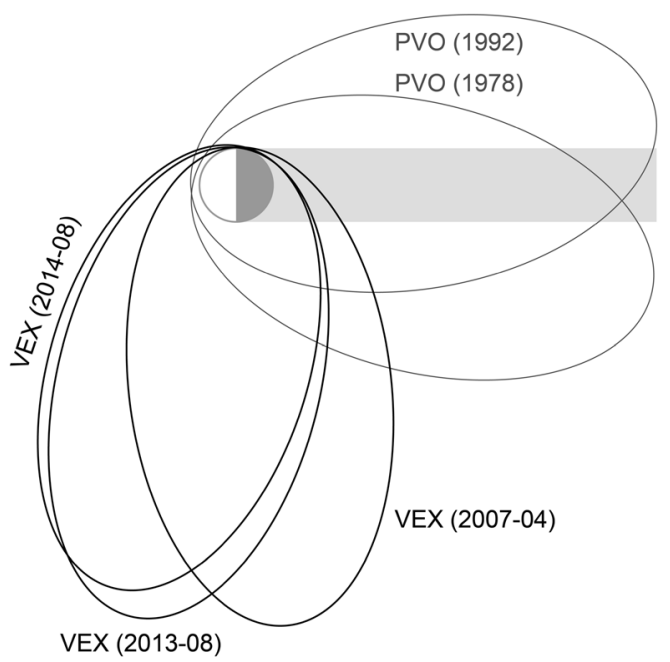

The PVO was launched in 1978 and the orbit coverage is shown in Fig. 3. This mission covered the noon and midnight regions down to $\sim 150 \mathrm{~km}$ altitudes and outward into the induced magnetotail (to $\sim 12 R_{\mathrm{V}}$ ) and out to the upstream interaction re- 
Fig. 4 Spatial coverage of the data (number of energy and mass spectra per $1000 \times 1000 \mathrm{~km}$ bin) of ion mass spectrometer (IMA), a part of ASPERA-4. One may see dense coverage of the IMA data in the near tail region and at low altitudes in the terminator region of Venus. The average bow shock (BS) and induced magnetosphere boundary IMB positions are also shown

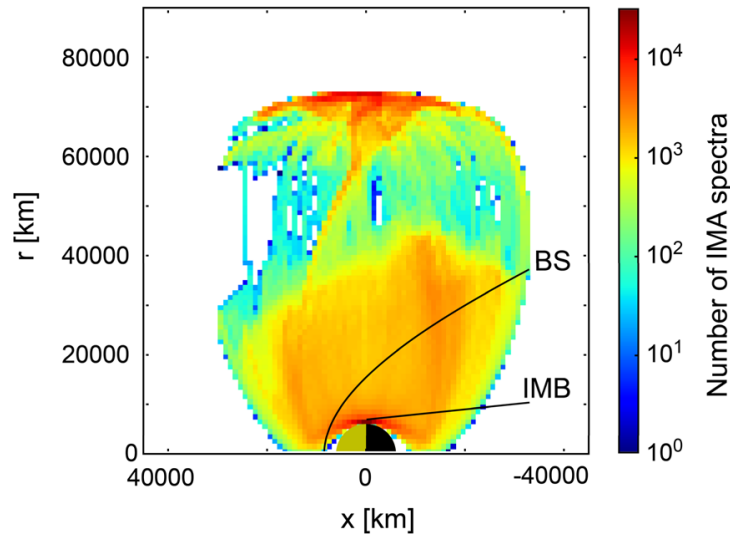

gion. The plasma investigation package onboard together with the magnetometer provided a fairly complete picture of Venus interaction with the solar wind. Key findings from PVO included; the verification of the absence of any global or crustal planetary fields of significance; the mapping of bow shock position and foreshock features, including their variation over the solar cycle; the investigation of the magnetosheath and magnetic barrier region, including the waves convected inward from the quasiparallel foreshock; the basic structure of the induced magnetotail; the ionosphere's solar maximum local-time/SZA dependences, including the ionopause boundary, ion composition, dynamics and magnetic fields; and unexpected features including ionospheric magnetic flux ropes and nightside ionospheric 'holes' (e.g. Russell and Vaisberg 1983; Luhmann 1986; Luhmann and Cravens 1991). In addition, while the energetic ion spectrometer did not have mass identification capability, it hinted, together with the suprathermal ion and Langmuir Probe measurements available, that ionized atmospheric constituents were escaping into the solar wind at possibly significant rates.

Almost 15 years later, the Venus Express mission carried extensive plasma instruments in order to complement the results from Pioneer Venus by making measurements in the polar and terminator regions as well as in the near tail, as indicated in Figs. 3 and 4.

\section{Key Features and Processes of the Venus-Solar Wind Interaction}

\subsection{Differences from the Earth}

Some readers may be more familiar with the Earth's solar wind interaction, illustrated in Fig. 5a. The near-Earth space environment is usually pictured with a bow shock standing upstream in the solar wind at $\sim 15$ planetary radii, produced by a magnetospheric 'obstacle' within which Earth's intrinsic dipole field dominates the structures and processes. In the region between the shock and the magnetosphere is the magnetosheath, where the incident solar wind plasma has been slowed and deflected around the magnetosphere, with an associated compression (pile-up) and draping of the frozen-in interplanetary magnetic field (IMF). This magnetosheath region is actually the part of near-Earth space that most resembles what is seen at Venus, where no significant planetary magnetic field exists.

A notable difference from the Earth's solar wind interaction concerns the spatial scales. For space plasma, several typical spatial lengths can be defined, and in each regime the dominant processes are different. If the spatial scale of a region, as in the magnetosheath, is much 
a)

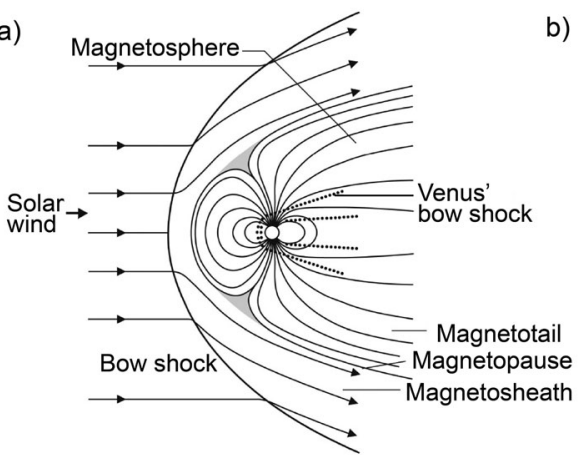

b)

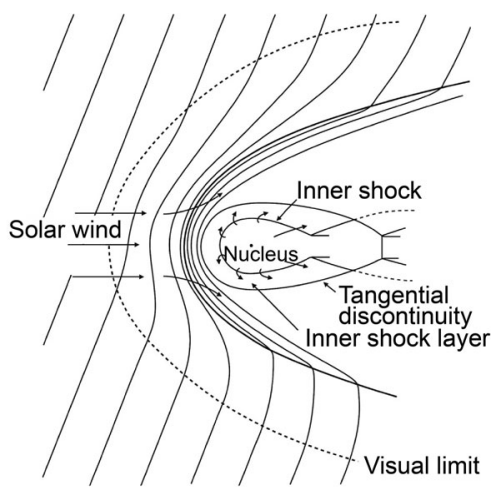

c)

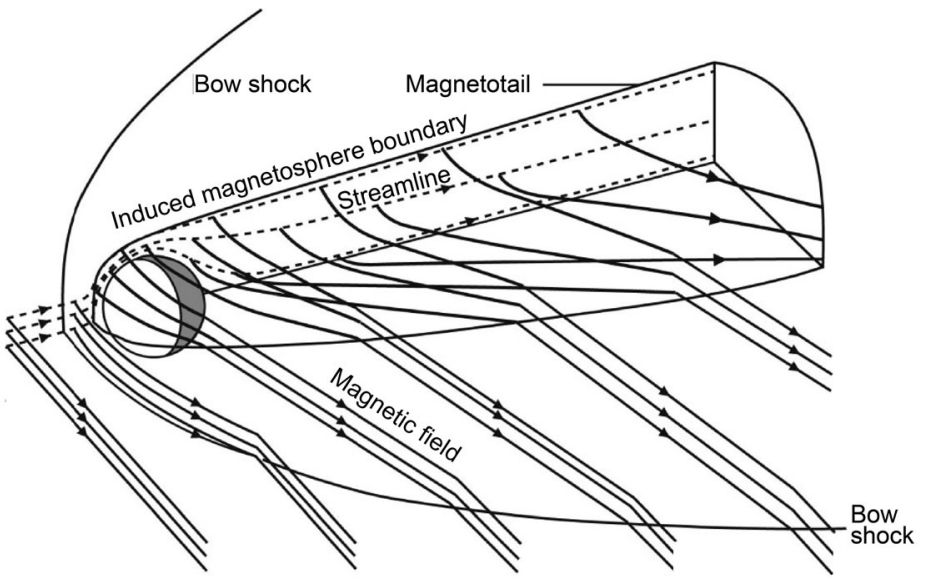

Fig. 5 Comparison of three different magnetospheres. The Earth's magnetosphere (a) is a typical example of an intrinsic magnetosphere. Venus magnetosphere is overlaid as a dashed line to illustrate the difference in size (adopted from Luhmann 1991a). A cometary induced magnetosphere is characterized by the draping of the solar wind magnetic field (b) introduced by the mass loading process (adopted from Russell et al. 2016). The induced magnetosphere of Venus (c) is a typical example of a magnetosphere of unmagnetized atmospheric object (adopted from Saunders and Russell 1986). Solar wind interaction to Mars exhibits similar interaction to Venus

larger than the ion gyroradius, the plasma behaves like a fluid. In this regime, the magnetohydrodynamic (MHD) approximation is reasonable for describing the physics. Where the spatial scale becomes smaller than the ion gyroradius, the ions show particle-like, kinetic behaviors, while the electrons still behave like a fluid. This is the hybrid regime. If the scale goes below even the electron gyroradius, the electrons also exhibit kinetic effects and the region may require a full kinetic treatment. Whereas at Earth, MHD treatments are often sufficient, hybrid descriptions are sometimes required for Venus. In particular, because of the close involvement of the heavy atmospheric ions in the solar wind interaction, which can have gyroradii on the order of the planetary radius in the near-Venus space environment, the discussion often includes both kinetic and fluid concepts together.

These basic differences from the Earth's solar wind interaction, in both basic physical setting and scale, lead to the dominance of physical processes at Venus not usually prominent in descriptions of intrinsic magnetospheres. Foremost among these are those involved in transfer of energy and momentum from the solar wind to the planetary atmosphere. These 
invoke the comet-like aspects of the situation at Venus (next section), and make its atmosphere especially important in defining the complete picture of its space environment.

\subsection{Differences from Comets and Mars}

Because of the lack of the significant planetary magnetic field but with sufficient atmosphere, Venus is categorized as an "unmagnetized atmospheric body". Active comets and Mars are in the same category. The interaction is characterized by a "induced magnetosphere" (Sect. 6). The features and processes characterizing the induced magnetosphere are often compared to those of a comet (cf. Fig. 5).

The appearance of an induced magnetosphere is often described by the term "draping". When the magnetized solar wind is slowed by the localized production of additional ion mass around a comet, where a gaseous atmosphere is produced and ionized as it approaches the Sun, the interplanetary magnetic field lines encountering the gas cloud pile up at the site of mass production while their ends, far from the comet, continue anti-sunward in the unperturbed solar wind flow. The associated magnetic field configuration in Fig. 5b was originally introduced by Alfvén (1957). This cometary interaction image also represents to some extent what is happening around Venus. It also introduces the concept of mass loading of the solar wind plasma by a source such as a planetary atmosphere. Because the solar wind approaches unmagnetized Venus so closely that it penetrates its upper atmosphere, the cometary picture is useful in describing the solar wind interaction. But it is not the entire picture.

The element of the induced magnetosphere at Venus that is missing from the purely cometary picture in Fig. $5 \mathrm{~b}$ is the additional local field distortion produced by currents induced in the planetary ionosphere. Fig. 5c shows the Venus counterpart of Figs. 5a and 5b, based on existing spacecraft measurements. In the same way that currents on the surface of a spherical conductor in a time-varying uniform magnetic field produce an opposing induced dipole field preventing penetration of the external field, currents generated in Venus' ionosphere produce fields that to first order exclude the IMF (e.g. Vaisberg and Zeleny 1984; Dubinin et al. 2013a). The fields of these ionospheric currents combine with the external magnetic field to produce the piled-up and draped magnetosheath-like fields that deflect the solar wind plasma around the 'obstacle': the main ionosphere and body of Venus. Mass loading by ion production in the upper atmosphere then occurs mainly on the draped magnetic fields closest to this ionospheric obstacle, which then slip over the obstacle and into the wake to form a comet-like induced magnetotail. Unlike an intrinsic planetary magnetosphere, the induced magnetosphere of Venus is thus highly tied to the IMF orientation, with the entire structure rotating with the perpendicular (to the incident flow) interplanetary field component, and sensitive to mass production by ionization of the atmosphere in which it is embedded.

Venus-solar wind interaction is quite similar to that with Mars. Mars has no intrinsic magnetic field, and therefore the ionosphere is interacting with the solar wind directly. Therefore, the similar induced magnetospheres are formed (e.g. Bertucci et al. 2011). On the other hand, the main difference in between is, again, the spatial scales. Due to the different size of the planets and the weaker interplanetary magnetic field, Mars shows more "particle"-like interaction. In addition, Mars has the localized magnetic field (also called as "magnetic anomaly") (Acuña et al. 2001), where the fluid approximation cannot be applied any more. 
a)

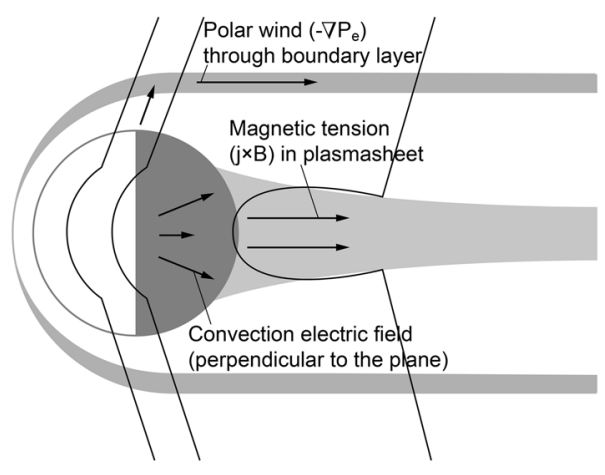

b)

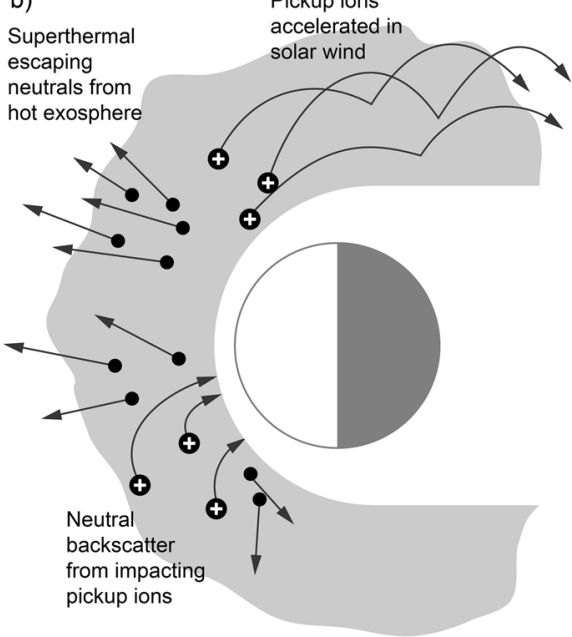

Fig. 6 Both fluid (a) and kinetic (b) processes play a role in the solar wind energization of the Venus atmosphere. (a) Fluid processes include acceleration by a parallel electric field caused by pressure gradients and the $\boldsymbol{j} \times \boldsymbol{B}$ force. (b) Pickup ions (figure redrawn based on Luhmann and Bauer 1992) on the other hand can be modeled as single test particles in the particle modeling perspective

\subsection{Acceleration Processes}

The plasma environment in the Venusian induced magnetosphere is characterized by certain forms of planetary particle acceleration and energization. One key outcome of the direct interaction of the solar wind with the atmosphere is ionospheric outflow. The solar wind plasma above the dayside flow deflection boundary can add to the photo-production of ionospheric ions there by the collisional processes of charge exchange (between the solar wind protons and upper atmosphere neutrals) and solar wind electron impact ionization, enhanced by the electrons' post-bow shock heating.

While the deflected and mass loaded solar wind plasma slows down close to Venus, the ions in the upper atmosphere can be accelerated (sometimes to $>\mathrm{keV}$ energies) via electromagnetic forces. Two different perspectives (Fig. 6) can be used to describe this process, depending on conditions and location. The combined solar wind and planetary plasma can be regarded as behaving like a fluid, described by MHD forces through the momentum equation (Dubinin et al. 2011):

$$
m \frac{d \boldsymbol{v}}{d t}=q\left((-\boldsymbol{v} \times \boldsymbol{B}) \times \boldsymbol{B}+\frac{\boldsymbol{j} \times \boldsymbol{B}}{n_{e} e}-\frac{\nabla P_{e}}{n_{e} e}\right)
$$

where the usual notation is used: $m$ is the ion mass, $\boldsymbol{v}$ is the bulk (fluid) velocity, $q$ is the ion charge, $n_{e}$ is the electron number density, $e$ is the elementary charge, $\boldsymbol{B}$ is the magnetic field vector, $\boldsymbol{j}$ is the electric current density (also describable by the curl of $\boldsymbol{B}$ ). Alternatively, a more kinetic-view of the planetary ion acceleration can be adopted where the particles are treated as single 'test' particles following the Lorentz force:

$$
m \frac{d \boldsymbol{v}_{p}}{d t}=q\left(\boldsymbol{E}+\boldsymbol{v}_{p} \times \boldsymbol{B}\right)
$$

where in this case $\boldsymbol{v}_{p}$ is the particle velocity and the fields $\boldsymbol{E}$ and $\boldsymbol{B}$ are assumed to be determined by background or external sources. 
In general, the use of these different treatments and their consequences depends on the circumstances of solar wind conditions and ionization processes. The fluid version is appropriate when and where the planetary ion density is large and its addition to the solar wind is a substantial contribution, while the single particle/kinetic approach is useful for understanding the acceleration and behavior of the planetary ions produced in the uppermost atmosphere where the magnetic field and convection electric field $(\boldsymbol{E}=-\boldsymbol{v} \times \boldsymbol{B})$ of the solar wind and magnetosheath mainly determine the forces. Ions treated using the second approach are often referred to as 'pickup ions' and the process as 'ion pickup' (e.g. discussion in Luhmann et al. 2006). Venus has an extended atmosphere, called an exosphere (or corona), that is mainly composed of atomic $\mathrm{H}$ and $\mathrm{O}$ extending to altitudes $>1 R_{\mathrm{V}}$ (Futaana et al. 2011 and reference therein). In this environment, the newly born ions are nearly at rest in the Venus frame, but the convection electric field can accelerate them up to twice the solar wind velocity for conditions where the solar wind velocity and IMF are perpendicular, regardless of the mass of the ions. With typical solar wind speeds of $\sim 400 \mathrm{~km} / \mathrm{s}$ this implies the production of $>60 \mathrm{keV} \mathrm{O}^{+}$ions.

Returning to the fluid picture and the fluid momentum equation, each term represents a macroscopic force on a fluid parcel that may have accumulated (ion) mass as it traveled through the Venus atmosphere. One term of particular interest is the $\boldsymbol{j} \times \boldsymbol{B}$ term. When the induced magnetosphere is produced by the solar wind, the shape of the magnetic field is draped as described above. Under this configuration the field has curvature, and there is an associated current given by Ampere's law: $\boldsymbol{\nabla} \times \boldsymbol{B}=\mu_{0} \boldsymbol{j}$. At the draping 'poles' and in the wake where the maximum field curvature exists (see Fig. 6a), the current is perpendicular to the $\boldsymbol{B}$ field, and the resulting $\boldsymbol{j} \times \boldsymbol{B}$ force exerted on the local plasma is tailward. The $\boldsymbol{j} \times \boldsymbol{B}$ force may play a significant role on the planetary ionospheric plasma escape from the Venus ionosphere through the solar wind wake, a concept that will come up again later in this paper.

Another force in the fluid equation is the electric field parallel to the magnetic field, $\boldsymbol{E}_{\|}$. However, such parallel electric fields are generated (or maintained) only under specific conditions because the accelerated plasma can generate electric fields in the opposite direction, diminishing the original field. Hartle and Grebowsky (1995) suggested that polarization electric fields played an important role in the nightside ionospheric outflow at Venus. In this case, the parallel electric fields are produced by pressure gradients and the gravitational separation of ions and electrons along the draped field lines.

\subsection{Energy and Momentum Transfer}

Even the simple difference between the dayside and nightside production rates of ionospheric ions in the main ionosphere can lead to a nightward flow due to thermal pressure gradients, without consideration of solar wind interaction influences (e.g. Miller and Whitten 1991). However, this process does not accelerate the oxygen ions to greater than escape velocity; the bulk of the oxygen ions thus stay in the ionosphere, with the dayside merely supplying the nightside. However, around several hundred $\mathrm{km}$ altitudes where collisions between particles become significant, energy and momentum can be directly exchanged between solar wind and planetary ions where solar wind deflection does not exclude the former. Incoming solar wind plasma particles with up to $\sim \mathrm{keV}$ energy can transfer energy to the relatively stationary upper atmospheric particles. This may be an especially effective energy transfer process near the magnetic draping poles of the induced magnetosphere, where the solar wind particles can access the upper atmosphere more easily that at lower magnetic 
latitudes (Lundin et al. 2011). Under such circumstances, it is appropriate to introduce a collision term in the fluid momentum equation above, describing these additional friction-like forces.

Another mode of energy transfer is via the process of magnetic reconnection, where a topological change occurs in the magnetic field due to localized diffusion in a region where magnetic shear is present. Even though the magnetic field around Venus is much different than in the Earth's magnetospheric interaction, the existence of reconnection in the draped fields, especially in the induced magnetotail and wake, can occur. Observations of apparent reconnection signatures there have been reported based on MAG and ASPERA-4 measurements (Volwerk et al. 2009; Zhang et al. 2012). Reconnection effectively converts magnetic energy to particle energy (both thermal and bulk). The inferred Venus magnetotail reconnection involves the disconnection of a plasmoid or a section of the plasma sheet separating the induced tail lobes - which results in a bursty flow of plasma sheet material down the wake as in the cases of the other planets. The induced magnetotail reconnections may thus enhance escaping ionospheric ion fluxes. Dayside reconnection also appears to occur with the passage of short-duration rotations/flips of the IMF orientation (e.g. as in IMF sector boundary crossings) (Vech et al. 2016), especially when they are associated with solar wind compressions. This process must re-configure the whole induced magnetosphere in a short time scale, with an associated acceleration of dayside ionospheric particles away from the planet at the interface between the old and new external field orientations. Edberg et al. (2011) showed these encounters resulted in statistical increases of the heavy ion escaping flux, presumably in association with the reconnection process.

Fluid shear instabilities like the Kelvin Helmholtz $(\mathrm{K}-\mathrm{H})$ instability are also expected to occur at the boundary between the ionospheric and solar wind (magnetosheath) plasmas, analogous to what happens at the magnetopause at Earth. In the standard picture of this process for Venus, large scale waves grow in the boundary layer between the magnetosheath and ionosphere at the flanks of the interaction, where they evolve into vortexlike structures that break away. As a result, the plasmas are co-mingled, and energy of the faster (solar wind/magnetosheath plasma) flow is transferred to the slower (planetary plasma) flow. Numerical simulations (e.g. Terada et al. 2002; Amerstorfer et al. 2007; Biernat et al. 2007) predicted the growth of the K-H instability at Venus. It may be that the 'bulk' escape of ionospheric ions suggested by the boundary plasma 'clouds' identified by Brace et al. (1982a) in PVO ionospheric boundary data are one result (Pope et al. 2009).

\section{An In-Situ Observer's Perspective of Near-Venus Space}

A typical time series of plasma and field data obtained on VEX is shown in Fig. 7. Approaching Venus from the Sun, the first distinct feature encountered is the bow shock, at which solar wind plasma is heated and decelerated. Crossing the bow shock the fluxes of energetic electrons, with energies of tens $\mathrm{eV}$, increase and the solar wind ions are clearly heated (Bertucci et al. 2011). The bow shock position changes with the solar cycle. At the terminator the shock, distance changes from $2.40 R_{\mathrm{V}}$ (Venus radii) during solar maximum to $2.14 R_{\mathrm{V}}$ during solar minimum, but VEX observations show that the subsolar shock is detached from the planet even for solar minimum conditions (Zhang et al. 2008a). There are also structures and phenomena observed outside the bow shock, in the 'foreshock', in association with the themalization processes occurring there.

Passing through the bow shock and entering the magnetosheath, greater levels of magnetic field fluctuations are seen. Wave-particle interaction is an important process for the 


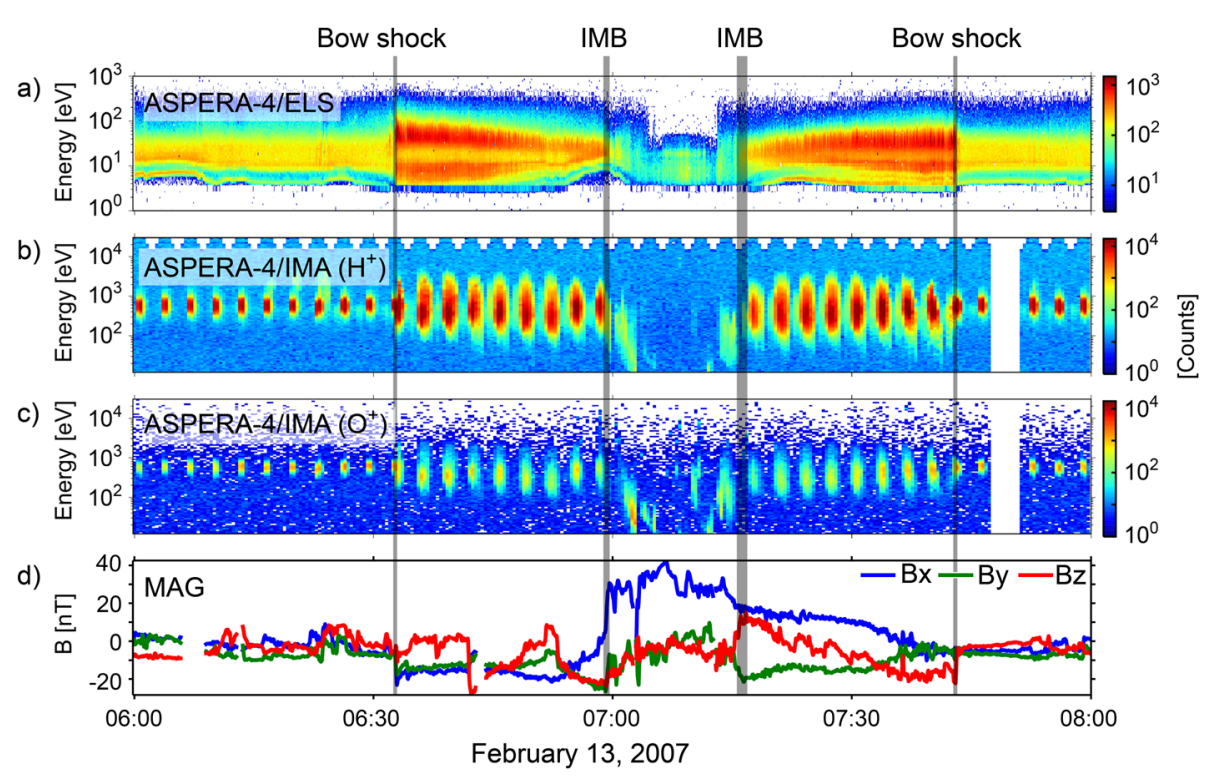

Fig. 7 Typical time series of the particle and the magnetic field data along the VEX orbit. (a) Electron spectra measured from ASEPRA-4/ELS, (b) proton spectra from ASPERA-4/IMA, (c) heavy ion spectra from ASPERA-4/IMA, and (d) magnetometer (MAG) data are shown. The bow shock (BS) and induced magnetosphere boundary (IMB), marked by vertical back lines, can be clearly identified in the ASPERA-4 and MAG data. At the bow shock the density of energetic electrons $(>50 \mathrm{eV})$ increases as seen in panel (a). The simultaneous increase of the proton temperature can be seen in panel (b). In panel (c), though a crosstalk from intense proton counts are seen in particular for the outside of the IMB, the oxygen ions of planetary origin can be seen inside the IMB. The hot electrons are substantially reduced inside the IMB. Simultaneous increase of the magnetic field strength (bottom panel) happens at the IMB. All the ASPERA-4 and MAG data is archived under the Planetary Science Archive (PSA) hosted by European Space Agency (http://www.rssd.esa.int/index.php?project=PSA)

thermalization of the solar wind plasma in the magnetosheath. The observed level of fluctuation depends strongly on the shock normal angle (Luhmann et al. 1983), which affects both the strength and the properties of the waves (Du et al. 2009). The most turbulent magnetosheath is found behind a quasi-parallel shock. Waves behind a quasi-perpendicular shock are more coherent. Mirror mode waves were also identified in VEX observations (Volwerk et al. 2008a).

The magnetic field in the magnetosheath is observed to gradually build up as the shocked solar wind slows down, forming the magnetic barrier that constitutes the real obstacle, which deflects the solar wind (Russell et al. 1979a; Zhang et al. 2008b). The magnetic barrier is found at lower altitudes $(\sim$ a few $100 \mathrm{~km}$ ) during solar minimum compared to solar maximum. It is sometimes associated with plasma depletion analogous to a similar reduction in the subsolar plasma density in the Earth's magnetosheath (Martinecz et al. 2009). An identifiable upper boundary of the magnetic barrier is seen where the magnetosheath wave activity is suppressed. Often this suppression is accompanied with a sudden change in the magnetic field components consistent with an increase in draping (Zhang et al. 2008b). This boundary is commonly referred to as the magnetic pileup boundary (MPB) (Kallio et al. 2008). At the subsolar point it is found at $300 \mathrm{~km}$ whereas it moves to an altitude of $\sim 1000 \mathrm{~km}$ at the terminator. 
Fig. 8 VEX measurements of the positions of three different plasma boundaries shown in a cylindrical coordinate system. Data show the bow shock (red), the induced magnetosphere boundary (green; equivalent to the upper mantle boundary) and the ion composition boundary (blue; equivalent to the lower mantle boundary) (adopted from Martinecz et al. 2009)

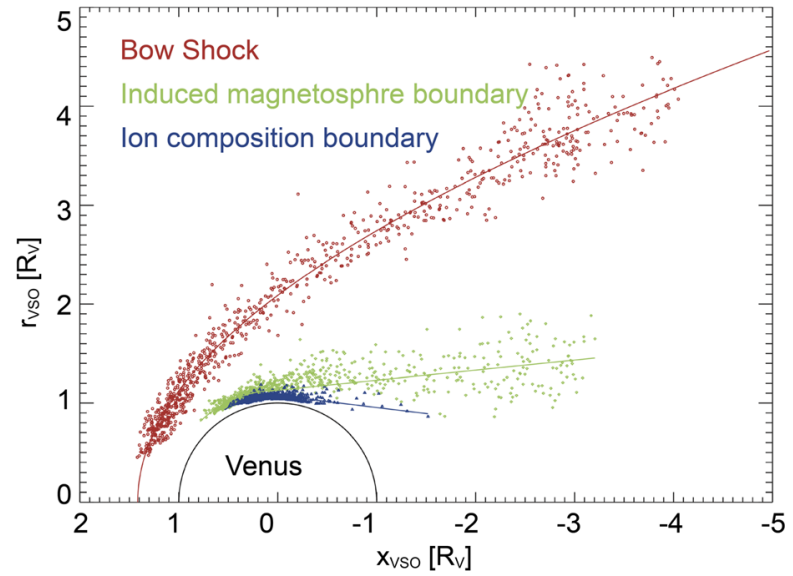

From a particle point of view the magnetic barrier overlaps a transition region, called the mantle, where a mixture of solar wind ions and planetary ions is observed (Martinecz et al. 2009). The upper boundary of the mantle is characterized by a sudden and strong decrease in energetic electrons. This upper mantle boundary is also called the induced magnetospheric boundary (IMB). Figure 8 shows observations of the bow shock and the upper and lower mantle boundaries.

During solar maximum, the lower boundary of the magnetic barrier forms the ionopause. The ionopause itself is the upper boundary of the ionosphere and is located where the magnetic pressure and ionospheric thermal pressure balance. It is characterized by a sharp gradient in electron density (Martinecz et al. 2009) at solar maximum because the thermal pressure in the ionosphere is high enough compared to solar wind pressure to keep the ionopause above the collisional region below a few $100 \mathrm{~km}$ (Luhmann 1986). At solar minimum the ionospheric thermal pressure is most often not large enough to achieve an ionopause pressure balance above $\sim 200 \mathrm{~km}$. Under these circumstances, the magnetic barrier field diffuses inward (Zhang et al. 2008b), and the ionosphere becomes magnetized (see Sect. 6).

Based on particle data one can identify an alternative boundary separating the ionospheric plasma from the shocked, magnetized plasma in the magnetosheath (Martinecz et al. 2008). This has been referred to as the Ion Composition Boundary (ICB) and manifests itself in the observations as a sudden disappearance of magnetosheath protons and appearance of lowenergy planetary ions. The ICB is the lower boundary of the mantle region. On the dayside, the ICB should then approximately correspond to the ionopause.

Another boundary, more or less coinciding with the ICB and the dayside ionopause, is the photoelectron boundary (PEB). Photoelectrons in this context are electrons resulting from the ionization of atomic oxygen and carbon dioxide in the Venus atmosphere. If the energy resolution of the electron instrument is high enough they can be seen as distinct peaks in an electron energy spectrum (Coates et al. 2008). The electron spectrometer on VEX resolved the peaks and the photoelectron signature was seen up to a solar zenith angle of $97^{\circ}$, just like at Earth (Coates et al. 2008). On Venus, the observed photoelectrons are believed to result mainly from ionization of oxygen (Coates et al. 2008, 2011). The photoelectrons sometimes escape along the magnetic field lines and are occasionally found far from the ionosphere indicating distant magnetic connections to their dayside source region (Tsang et al. 2015; Coates et al. 2015).

Although these many terms and features are useful to describe the dayside boundary of the Venus obstacle, there are many cases when the boundary crossings cannot be identified 
in the post-terminator region; in general, boundary crossing in the post terminator region is more gradual than that for the dayside crossings. The antisolar pressure-gradient supplied nightside ionosphere and near-Venus wake that was observed by PVO at solar maximum exhibited features such as ionospheric holes and tail rays (Brace et al. 1982a, 1987) that are still not fully understood. The situation for low solar activity at low altitudes is still relatively unexplored. Although Venus Express' high latitude measurements have added further information, the different solar cycle phases dominating the PVO and VEX missions complicates the comparisons of their results, some of which are described in more detail below.

Proceeding into the solar wind wake of the Venus interaction, one finally reaches the magnetotail (see Fig. 5c), which consists of two magnetic lobes with opposite polarity that unlike the Earth's intrinsic field magnetotail, instead have a typical east-west separation akin to the magnetosheath draping pattern. A plasma sheet, where planetary ions are energized to hundreds of $\mathrm{eV}$ and are seen escaping downstream, separates the two lobes in a plane normally in the noon-midnight meridian. The basic geometry of these features for Venus is a result of average interplanetary fields lying close to the Venus orbital plane. In fact, this picture dynamically adjusts to all of the internal and external influences that create it, from the ionizing solar flux to the solar wind pressure and IMF orientation. It can be imagined that if one could in fact image the Venus-solar wind interaction from afar, it would indeed resemble a comet.

All of these observed features and phenomena deserve much more detailed descriptions that are difficult to cover in a review of reasonable size. Thus, in what follows several areas are highlighted with an emphasis on what VEX has revealed since the already well-reviewed earlier missions.

\section{Details of the Bow Shock and Upstream Region}

\subsection{Location and Structure of the Bow Shock}

The location of the bow shock changes with the extreme ultraviolet (EUV) radiation from the Sun. Increased EUV radiation leads to heating of the upper atmosphere/exosphere and an increased scale height. The ion production is enhanced due to both the associated increased photoionization rates and the larger scale height. Stronger mass loading of the solar wind plasma by these upper atmospheric ions then pushes the bow shock outward.

The shock is a magnetosonic wave propagating towards the Sun, standing in the solar wind flow (Russell et al. 1988) and the location therefore depends on the solar wind velocity compared to the wave velocity, that is, the solar wind (magnetosonic) Mach number. The Mach number depends on the magnetic field strength and the wave propagation velocity depends on the direction of propagation with respect to the ambient field, which means the bow shock location is sensitive to magnetic field orientation and strength (Russell et al. 1988). PVO made observations of the bow shock during an entire solar cycle. However, due to the orbit geometry, VEX is much better suited to observe the bow shock away from the terminator plane: close to the subsolar point as well as in the near tail. The spacecraft crosses the bow shock at a highly inclined angle in a large solar zenith angle range, providing well-defined shock crossings (Zhang et al. 2008a).

An average bow shock shape can be obtained from observed bow shock crossings identified in magnetic field or particle data. The locations of the crossings are fitted using a given model. A common approach is to use a conic section, either using the center of 

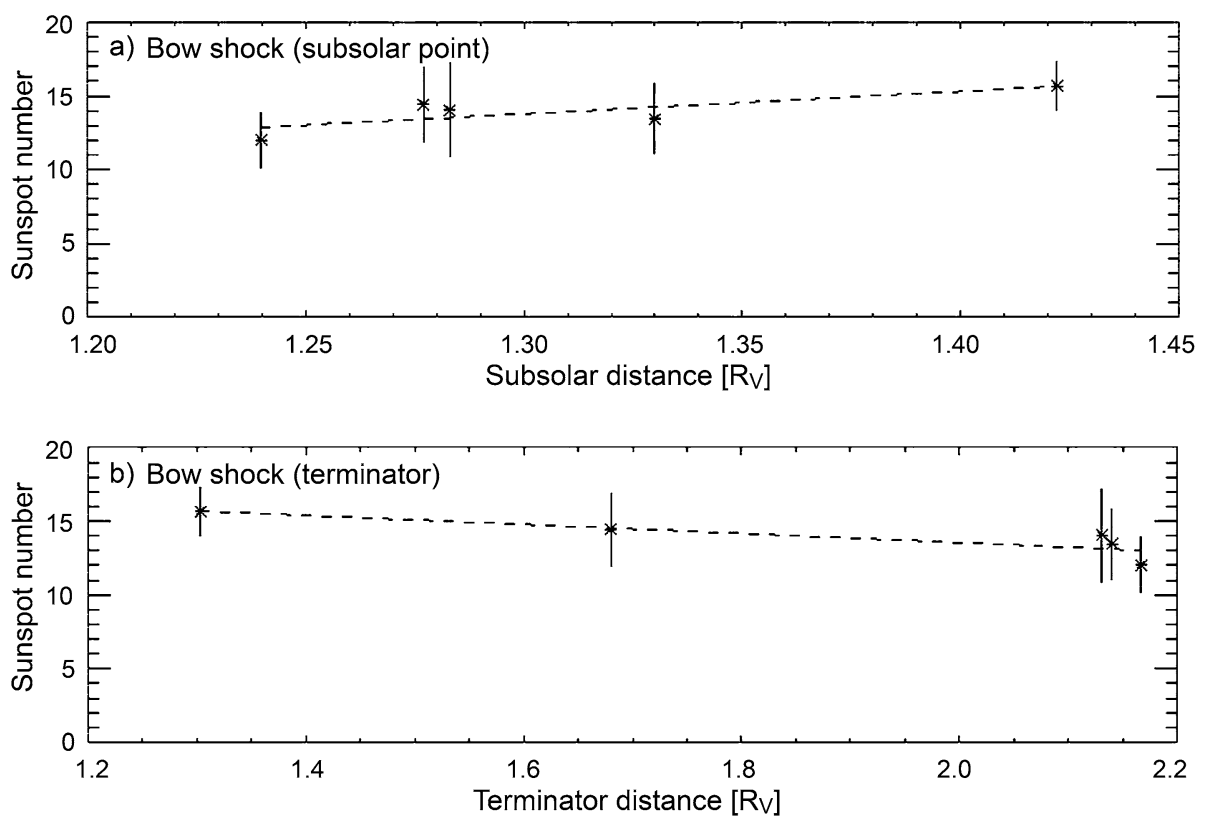

Fig. 9 The subsolar and terminator distances to the bow shock vary with the sunspot number showing the sensitivity of the boundary to solar conditions. While the subsolar distance increases with the sunspot number the terminator distance decrease meaning the shape of the bow shock changes as the sunspot number changes (adopted from Whittaker et al. 2010)

the planet as focus (e.g. Zhang et al. 2008a) or letting the focus point along the SunVenus be a free parameter (e.g. Martinecz et al. 2008). However, a parabola also gives an acceptable fit (Whittaker et al. 2010). The models are valid for solar zenith angles up to $90-120^{\circ}$ and they are stitched together with an asymptotic shock cone (Mach cone) on the nightside (e.g. Zhang et al. 2008a). Different studies report slightly different results, depending on the spacecraft (Venera-9/10, PVO or VEX), the type of data used and the choice of time period and fitting method. For solar minimum conditions, the terminator shock distance estimates ranges from $1.98 R_{\mathrm{V}}$ to $2.17 R_{\mathrm{V}}$ (Martinecz et al. 2008; Whittaker et al. 2010) and the subsolar shock distance ranges from $1.23 R_{\mathrm{V}}$ to $1.42 R_{\mathrm{V}}$ (Whittaker et al. 2010). Regardless of which model is used the subsolar bow shock is always well above the obstacle and hence, the shock is completely detached even at solar minimum (Zhang et al. 2008a).

Investigating the average solar conditions during the different time periods considered in different bow shock location studies, Whittaker et al. (2010) find linear relationships between sunspot number and subsolar and terminator shock distances. The terminator shock distance decreases with an increasing sunspot number, a dependence previously seen in PVO data (Phillips and McComas 1991). The subsolar shock distance, however, increases when the sunspot number increases. Hence, the shape of the bow shock is sensitive to the sunspot number, at least during low solar activity conditions (Fig. 9). The subsolar shock distance is anti-correlated with solar EUV (F10.7 proxy) while no effects are seen at the terminator (Whittaker et al. 2010).

The shape should change more over an entire solar cycle. PVO observations showed a change of the terminator shock distance from $2.45 R_{\mathrm{V}}$ during solar maximum to $2.13 R_{\mathrm{V}}$ at 
solar minimum (Russell et al. 1988). The variation in the solar EUV is a likely explanation (see above). Hence, it is not so surprising that the terminator shock distances observed by VEX are quite different. According to VEX measurements the bow shock is located further out during solar maximum, but the terminator shock distance of $2.14 R_{\mathrm{V}}$ is comparable with the solar minimum value determined by PVO (Shan et al. 2015). However, the solar maximum experienced by VEX was very weak, as inferred from the F10.7 flux and sunspot number, and the solar minimum extremely long and weak. VEX observations show that the terminator shock distance changes from 2.08 to $2.14 R_{\mathrm{V}}$ and the subsolar shock distance from 1.36 to $1.46 R_{\mathrm{V}}$ during the solar cycle in the model used by Shan et al. (2015).

The size and shape of the bow shock depends not only on the size of the obstacle but on also to what extent the solar wind is deflected around the planet. Hence, bow shock observations can be used to indirectly infer that entry of solar wind particles into the induced magnetosphere is minimal (Zhang et al. 2007a). Parametric models of the average bow shock position often assume symmetry with respect to the Sun-Venus line. This is not entirely true (already known from Venera-9 and 10 observations; Romanov et al. 1978). When the interplanetary magnetic field is perpendicular to the solar wind flow, a cross section of the shock in the terminator plane is in fact an ellipse (Alexander et al. 1986; Russell et al. 1988), with the major axis aligned with the solar wind convection electric field. This asymmetry is referred to as the (magnetic) pole-equator asymmetry. There is also a magnetic north-south asymmetry, where the bow shock is further out above the magnetic north pole, that is, where the convection electric field points away from the planet. An excess of pickup ions increases the size of the obstacle and causes the north-south asymmetry. It was recently demonstrated that the north-south asymmetry is larger for larger solar zenith angles but is observable even in the near-subsolar region (Chai et al. 2015). The pole-equator asymmetry is however not observed in the subsolar region. It is suggested that the pole-equator asymmetry is caused by the fact that the wave velocity depends on the angle of propagation with respect to the magnetic field (Romanov et al. 1978). Close to the subsolar point the distance to the bow shock is small and the asymmetry may not have time or space to develop.

The strength and direction of the interplanetary field indeed has a significant influence on the bow shock position. The shock terminator distance increases with the magnitude of the magnetic field (Chai et al. 2014). The shock size also depends linearly on the magnitude of the magnetic field component tangential to the shock surface. A direct effect of this relationship is a magnetic dawn-dusk asymmetry, which also has been reported (Chai et al. 2014) and which is consistent with simulations (Jarvinen et al. 2013). Depending on the sign of the magnetic field component along the Venus-sun line, the component tangential to the shock surface reaches the maximum value either on the magnetic dawn or on the magnetic dusk side.

Solar activity effects can sometimes lead to extreme situations. Both PVO and VEX observed bow shock crossings at extremely large distances from the planet (Russell and Zhang 1992; Zhang et al. 2008c). These anomalous shock locations are all attributed to low Mach numbers. Models show that at low Mach numbers the shock location becomes very sensitive to changes of the Mach number (Farris and Russell 1994; Verigin et al. 2004). Indirectly, sensitivity to the Mach number includes sensitivity to the interplanetary magnetic field direction since the magnetosonic velocity depends on the angle between the ambient magnetic field and the propagation direction. Hence, the Mach number depends on the angle between the shock normal and the magnetic field. VEX observations of a distant bow shock were made during the passage of an interplanetary coronal mass ejection (ICME) on September 10-11, 2006. The average magnetic field was higher than normal, leading to a decrease of 
the Alfvén Mach number and thereby also of magnetosonic Mach number. ICMEs substantially influence the bow shock position mainly in those cases when a magnetic cloud 'driver' with large field magnitude and low density is part of the disturbance (Vech et al. 2015).

The special solar wind conditions during the ICME encounter in September 2006, also led to an observation of a magnetic shock profile not previously seen (Balikhin et al. 2008). Subcritical (low Mach number) shocks are classified as dispersive or dissipative (Kennel et al. 1985). For a quasi-perpendicular dispersive shock, wave precursors are observed standing ahead of the shock ramp, while no such oscillations are seen in a dissipative shock. In the classical picture no oscillations are seen downstream of a subcritical quasi-perpendicular shock. The shock crossings observed on September 10, however, featured both wave precursors and a set of downstream oscillations, polarized along the mean field (Balikhin et al. 2008). A probable cause of the oscillations is that the ion distribution gyrates as a whole, resulting in a spatial variation of the ion pressure, which is compensated by the magnetic field to keep pressure balance. Gyrophase mixing eventually leads to decay of the oscillations and a thermalized ion population further downstream.

The increased mass-loading occurring when the solar EUV flux increases also makes the shock weaker. The magnetic field compression ratio decreases when the percentage of pickup ions in the background plasma increases (Lu et al. 2013). The compression ratio is defined as $B_{d} / B_{u}$, where $B_{d}$ and $B_{u}$ is the magnetic field strength downstream and upstream of the shock, respectively. The presence of mass loading makes the Venusian bow shock weaker than the Earth bow shock, an effect known since the first observations of PVO (Russell et al. 1979b).

Not only the location but also the shock structure and spatial scales change with upstream conditions. The Earth's bow shock has been extensively studied and multi-spacecraft missions make it possible to accurately separate spatial and temporal changes. The magnetic profile of the Earth bow shock is thus well known. The spatial extent of the so-called overshoot, which appears for supercritical shocks, is for most shocks about three times the upstream ion gyro radius (Livesey et al. 1982; Saxena et al. 2005). Using this assumption, the spatial scales of other layers within a shock can be estimated. An attempt to use VEX magnetic field data to investigate the spatial scale of the shock ramp at Venus shows that the typical width of the ramp is half the gyro radius of an upstream proton; the value is similar to what is observed at Earth (Dimmock et al. 2011).

\subsection{Foreshock Observations}

The region upstream of a bow shock, which is magnetically connected to the shock, is referred to as the foreshock (Eastwood et al. 2005). In the terrestrial foreshock, a plethora of different plasma waves are present, created when some part of the solar wind plasma is reflected at the shock and moves back upstream. The back-streaming energetic ions are classified into three categories depending on their distribution function: field-aligned, intermediate (gyrating) and diffuse. The field-aligned ions flow along the magnetic field while the intermediate population consists of ions streaming with a finite angle with respect to the magnetic field and the diffuse population is nearly isotropic in a wide energy range.

Observations in the foreshock of Venus reveal ion populations similar to those seen in the terrestrial foreshock. The observed particles flow away from the shock more or less parallel to the magnetic field. The variation in the parallel velocity component $\left(\delta V_{\|}\right)$is small, which is consistent with specular reflection of ions at the shock (Yamauchi et al. 2011). The ions observed in foreshock at Venus are quite different from what is seen at Mars. There the intermediate gyrating component seems to be absent and instead two other types of gyrating 
Fig. 10 Schematic sketch of the formation of a hot flow anomaly. An HFA is a dynamic phenomenon characterized by a magnetic field cavity surrounded by enhanced magnetic fields and hot deflected plasma. The convection electric field points towards the low field region, at least on one side of the HFA. Figure is redrawn based on Collinson et al. (2012a)

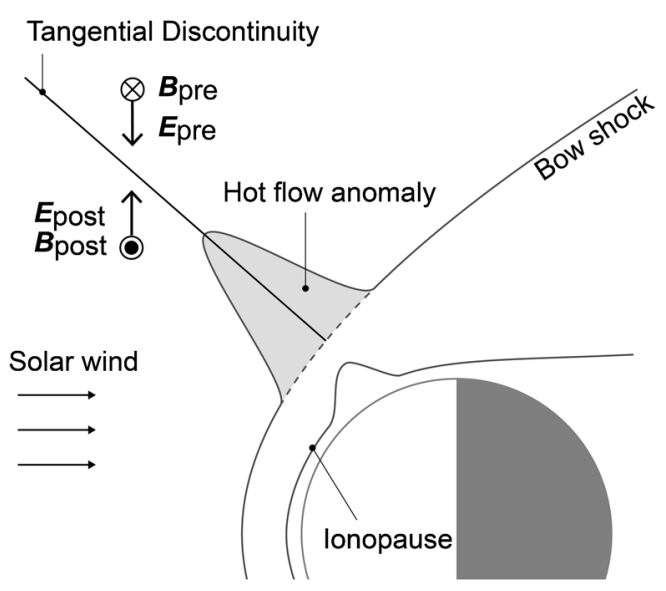

protons are observed (Yamauchi et al. 2011). One possible explanation for the difference between the planets may be the high amount of cold ions present close to the bow shock at Mars. The Martian bow shock is also so small compared to relevant gyro radii that the curvature cannot be ignored.

When a tangential discontinuity in the solar wind interacts with a bow shock, a so-called hot flow anomaly (HFA) can form. HFAs appear as strongly deflected hot plasma regions, often with a noisy but depressed magnetic field, surrounded by compressed shocked plasma (Schwartz et al. 2000). They were first discovered upstream of the Earth bow shock in mid1980s (Schwartz et al. 1985; Thomsen et al. 1986) and were initially believed to be transient phenomena with no significant global effects. Later Sibeck et al. (1998, 1999) presented evidence that HFAs are very dynamic phenomena that have a profound influence on the interaction between the solar wind and the magnetosphere. A schematic sketch is shown in Fig. 10.

HFAs are observed not only at Earth, but also by the Cassini spacecraft (Matson et al. 2002) in the Kronian foreshock (Masters et al. 2009). Øieroset et al. (2001a) reported signatures consistent with HFAs at Mars. When the MESSENGER spacecraft (Solomon et al. 2001) en route to Mercury, in June 2007, performed a gravity assist at Venus, it recorded two events consistent with HFAs (Slavin et al. 2009). The observations show enhanced magnetic fields surrounding regions where the field is very low except at the very center of the region. The convection electric field points towards the low field region, at least on one side, which is a requirement for HFA formation (Schwartz et al. 2000).

Later examples of HFA candidates at Venus include plasma data, and show increased electron densities and higher (pseudo) temperatures in the compressed regions surrounding the cavity, as well as a temperature increase in the core (Collinson et al. 2012a, 2014a). The resolution of the ion data is not high enough to capture the ion population inside the HFA, but ion observations show evidence of a deflection of the plasma flow. The estimated occurrence rate of HFAs observed in the Venusian foreshock is 1.2 per day. However, they are only detected for solar wind velocities $>370 \mathrm{~km} / \mathrm{s}$, and taking this into account the frequency increases to 4.2 per day when the solar wind velocity is high enough (Collinson et al. 2014a). Discontinuities in the form of current sheets in the solar wind at Venus are occurring with a rate of 3.5 per day (Zhang et al. 2008d), which matches the HFA frequency.

The HFAs observed at Venus are physically smaller than their terrestrial counterparts but they are larger compared to the size of the magnetosphere. The size is probably related to 
the time the anomaly has to grow, that is, a larger bow shock lead to larger HFAs. The local ion gyroradius is mostly likely also a factor determining the size (Collinson et al. 2014a).

The first observations of SLAMS (Short Large-Amplitude Magnetic Structures) in the Venusian foreshock may have been made with VEX (Collinson et al. 2012b). SLAMS are previously observed in the Earth's foreshock (Schwartz et al. 1992; Mann et al. 1994), but have also been detected at comet Giacobini-Zinner (Tsurutani et al. 1990) and at Jupiter (Tsurutani et al. 1993). At Earth they appear as monolithic spikes in magnetic field magnitude, with a compression ratio $\left(\delta B / B_{0}\right)$ of $2-5$ and duration of about 10 seconds (e.g., Schwartz et al. 1992). They are believed to originate from ULF waves, generated by a fieldaligned ion beam reflected at the bow shock. As the waves convect back towards the shock they encounter diffuse ions, the index of refraction changes and the waves become compressive and steepen. Their phase speed increase with amplitude, that is, their velocity relative to the planet decreases, and they may together build up the quasi-parallel bow shock.

So far only three potential SLAMS has been observed in the Venusian foreshock (Collinson et al. 2012b). Their characteristics are similar to SLAMS observed at Earth. Observations show spikes in the magnetic field with a compression ratio above 2 . They are lefthanded and elliptically polarized in the spacecraft frame, consistent with observations at Earth (Lucek et al. 2008).

\section{Inside the Induced Magnetosphere}

The obstacle in the induced magnetosphere is often regarded as the ionopause, where the external pressure (solar wind dynamic pressure, having been converted to magnetic pressure in the magnetic barrier) balances the thermal pressure of the ionosphere. The shape and location of the ionopause is characterized by the pressure balance.

$$
\frac{\rho v^{2}}{2}=\frac{B^{2}}{2 \mu_{0}}=n k T_{e}
$$

where $\rho$ is the solar wind mass density, $v$ is the solar wind speed, $B$ is the magnetic field strength, $n$ is the electron density in the ionosphere, and $T_{e}$ is the electron temperature in the ionosphere (the ion pressure can be neglected compared to that of the electrons).

PVO observations actually found two different states of the dayside interaction depending on where the pressure balance is achieved. If the pressure balance occurs above the collisional region (starting at about a few hundred $\mathrm{km}$ altitudes) there can be a relatively sharp boundary between the magnetic barrier fields and the ionospheric plasma, with thickness even down to a few $10 \mathrm{~s}$ of $\mathrm{km}$ (the order of an ion gyroradius there). Under these circumstances the ionosphere the magnetic field strength is very weak relative to that in the barrier region above it. Such ionosphere states are called "unmagnetized". Under the unmagnetized ionospheric state, some distinct, small scale magnetic field structures, resembling magnetic flux ropes, and of still uncertain origin, are seen (Russell and Elphic 1979). On the other hand, if the external pressure is high compared to the pressure in the upper ionosphere (frequently occurrence under solar minimum conditions) the nominal pressure balance occurs in the collisional region below a few hundred $\mathrm{km}$ altitude. Under these conditions, the external field appears to diffuse and convect downward into the ionosphere (Luhmann et al. 1984). This condition is called the "magnetized" ionosphere (Luhmann and Cravens 1991). The unmagnetized state occurs most frequently in the subsolar region (Luhmann and Cravens 1991). It should also be mentioned here that ionospheric flux ropes similar to those observed on PVO were also seen during the more weakly magnetized ionosphere passes on VEX (Wei et al. 2010). 

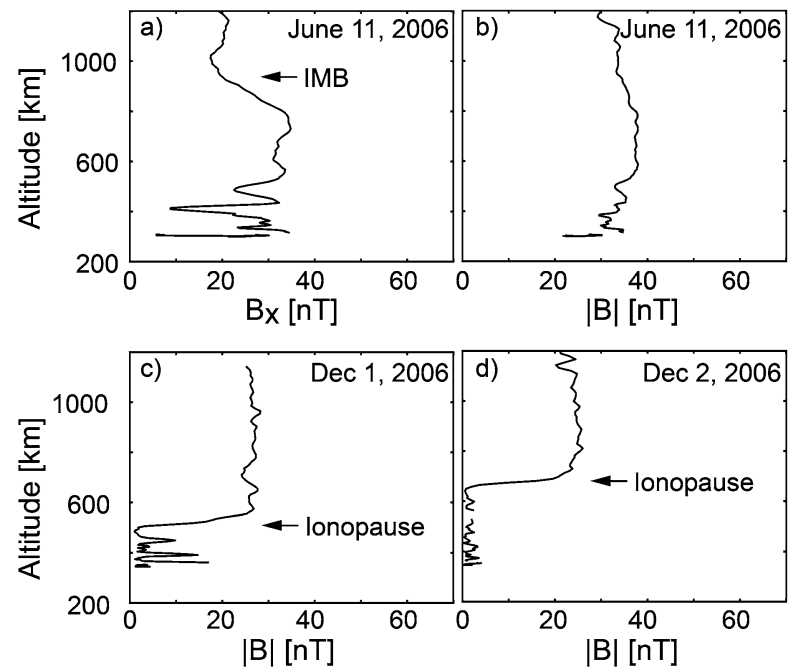

Fig. 11 Examples of the magnetic field observations near the induced magnetosphere by the VEX/MAG instrument (Zhang et al. 2008b). (a, b) A case of magnetized ionosphere (on 11 June, 2006), when the IMB formation is seen as a rather sharp changes of the $B_{x}$-component (a), without changing total field strength (b). At the lower altitude, the magnetic field is still intense, indicating that the ionosphere was magnetized. (c, d) Cases of unmagnetized ionosphere (on 1 and 2 December, 2006). The ionopause (IP) is formed as sharp decrease in the total field. The field strength is nearly zero, with sharp increases due to magnetic flux ropes, indicating the ionosphere was unmagnetized. The IMB was formed at the altutides higher than $1200 \mathrm{~km}$ during these days. Figures are after Zhang et al. (2008b), with reproduction from the data archived in ESA/PSA (http://www.rssd.esa.int/index.php?project=PSA)

The measurements by Venus Express probed the ionospheric magnetization states mainly around high northern latitudes of its periapsis during the cycle 23 solar minimum. This is a special location in that the incident solar wind pressure is applied at a large angle to the vertical there, and that it often where the draped external field develops its most extreme curvature before it sinks into the wake to join the magnetotail. Using the magnetometer (MAG) data in the very early phase of the VEX mission (April to August 2006), Zhang et al. (2008b) reported $95 \%$ of the orbits were in magnetized cases (Fig. 11). The other $5 \%$ were unmagnetized, but this happens only under the strong influence under disturbed solar wind conditions - an interesting finding considering that these periods exhibit highest dynamic pressures and the opposite effect occurs at small solar zenith angles. On the other hand, using a larger dataset (from June 2006 until 2009), Angsmann et al. (2011) found 58\% of all VEX orbits had ionospheres in the magnetized state based on MAG and electron (ELS) data. While these results are quantitatively different due to their use of different data sets and criteria, they agree that the northern high latitude ionosphere is frequently magnetized with large scale fields under the low solar activity conditions prevailing for much of the VEX mission.

Still unclear are the details of what ultimately happens to the ionospheric magnetic fields as they slip around and through the ionosphere. Some collisional dissipation certainly occurs in the atmosphere, but some calculations of the time scales for the decay of subsolar fields suggest they can last for minutes to hours (e.g. Luhmann and Cravens 1991). This implies there is a 'memory' of past penetrated interplanetary fields in the ionosphere (as at other weakly magnetized bodies, e.g. Bertucci et al. 2008) whose ultimate fate is unclear. There is also the question of what happens to the penetrated fields at the lowest 
altitudes. PVO, which had a mid-to-low latitude periapsis, and so regularly sampled the ionosphere down to $\sim 150 \mathrm{~km}$, obtained altitude profiles of the ionospheric fields that could be reproduced with 1D diffusion/convection calculations. However, the lower boundary assumption was found to be somewhat flexible in terms of allowing nonzero fields (e.g. Luhmann 1991b). Moreover, near-radial magnetic fields observed on PVO in the deep nightside ionosphere, together with ionospheric depletions that inspired the label 'ionospheric holes' (Brace et al. 1982b), have still undetermined roots. The main features of the holes are an enhanced magnetic field in the sunward/antisunward direction together with a depletion of the plasma density. The orientation of the magnetic field in a hole is consistent with draping of the interplanetary field. They are also characterized by low plasma $\beta$ and dual electron populations and they seem to occur in pairs with opposite magnetic field polarization (Brace et al. 1980, 1982b; Marubashi et al. 1985; Luhmann and Russell 1992; Hoegy and Grebowsky 2010). Observations made by VEX show what appears to be the tailward extension of such holes. Further out in the tail (1.2-2.4 $\left.R_{\mathrm{V}}\right)$ the size of the holes is larger and the magnetic enhancements weaker. In fact, for the holes to be observable in the tail the background magnetic field has to be weak, which may explain why only eleven event were detected in the VEX data from 2006-2012 (Collinson et al. 2014b). These observations raise the possibility that the dayside penetrated fields and fields in the nightside ionospheric holes fields may be connected, but the participation of the lower atmosphere and interior of Venus in the solar wind interaction is still largely unexplored (but see Villarreal et al. 2015). Even the existence of a weak internal dipole field has been proposed as an explanation of the holes (Knudsen et al. 1982), though the VEX low altitude field observations during the endof-mission phase are not consistent with the required polar surface fields (Zhang et al. 2015; Luhmann et al. 2015).

In general, the magnetic field draping around Venus can be regarded as a superposition of the interplanetary field and induced field components with different origins. Currents in the ionosphere are created as a response to temporal variations in the interplanetary magnetic field (Faraday induction, $\boldsymbol{\nabla} \times \boldsymbol{E}=-\partial \boldsymbol{B} / \partial t$ ). The flow of the solar wind past the planet also generates currents to keep the external field out of the conducting ionosphere (unipolar dynamo, $\boldsymbol{E}=-\boldsymbol{V}_{S W} \times \boldsymbol{B}$ ). The two resulting field configurations are referred to by their different magnetic field orientations as poloidal and toroidal, respectively (Sonett and Colburn 1967; Schubert and Schwartz 1969; Luhmann 1992; Dubinin et al. 2013a). Both induction mechanisms were initially discussed in the context of a possible induced lunar magnetosphere (e.g., Johnson and Midgley 1968), before any induced magnetosphere had been discovered.

The shielding of the interplanetary field provided by the induced currents is not perfect and magnetic field diffuses into the ionosphere. The parts of the field lines passing through the ionosphere are mass-loaded, which results in a further stretching of the magnetotail, when the mass-loaded section is slowed down compared to the solar wind flow (Luhmann 1992). At least at times there is a magnetic connection between the sunlit dayside ionosphere and the tail, proven by the observations of photoelectrons in the magnetotail (Tsang et al. 2015). These electrons result from photoionization of mainly oxygen on the dayside and they have characteristic energies, which makes them recognizable in electron measurements.

To distinguish a toroidal contribution from a poloidal is not as easy as one would imagine. Figure 12 shows sketches of the draped field for the two mechanisms ( $a$ and $b$ ). The pile-up of the magnetic field on the dayside occurs in both cases and it is only at relatively low altitudes and in the wake or near tail any differences can be observed. One possible signature of the toroidal field is the reversal of the cross flow magnetic field component. Such reversals are indeed seen in the $-E$-hemisphere (the hemisphere where 
a)

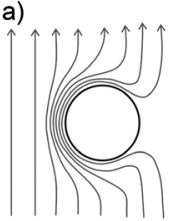

b)

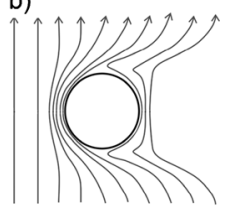

c)

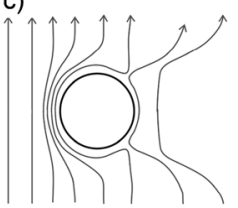

Fig. 12 Possible magnetic field topologies. Induced currents from a motional electric field result in the pattern shown in (a) while an induced dipole (Faraday induction) gives the topology seen in (b). Combining the two mechanisms gives (c) (after Dubinin et al. 2013a)

the solar wind convection electric field points towards the planet) (Zhang et al. 2010; Dubinin et al. 2013a). A poloidal component may instead be recognizable through reversals of the $B_{x}$-component (the component anti-parallel to the upstream plasma flow) in places where it is not expected from the general draping (cf. Fig. 12b). Dubinin et al. (2013a) presented both single events and statistics indicating a poloidal component manifested by such reversals of the $B_{x}$-component. Since signatures of both toroidal and poloidal fields exist it is natural to assume that both induction mechanisms are in play.

\section{Details of the Magnetotail}

The plasma and magnetic field properties of the comet-like Venus magnetotail were initially investigated by the Venera and PVO missions before VEX (e.g. Vaisberg et al. 1995), but each mission's in-situ view is always limited by orbital sampling and available instrumentation. In particular, while PVO was able to obtain a relatively detailed mapping of the Venus wake around its apoapsis at $\sim 12 R_{\mathrm{V}}$, and also near its periapsis in the nightside ionosphere, the important near wake region was almost unexplored until the arrival of VEX. In addition, the energetic ion spectrometer on PVO had both no mass identification capability and an upper energy cutoff at $\sim 8 \mathrm{keV}$ that was too low at the high energy end, and not designed for the low energy end, for capturing the planetary ions involved. With ASPERA-4 IMA's greater energy range of $\sim 10 \mathrm{~s}$ of $\mathrm{eV}$ to $\sim 25 \mathrm{keV}$, coupled with mass discrimination and the VEX sampling geometry, a much more complete picture of the Venus magnetotail has emerged.

\subsection{Magnetic Field Morphology}

The magnetic field in the two tail lobes is roughly parallel and anti-parallel to the Sun direction both in the distant tail (Yeroshenko 1979; Dolginov et al. 1981; Saunders and Russell 1986) and in the near wake region probed by VEX (Zhang et al. 2010). The cross flow component of the solar wind magnetic field in general keeps its orientation throughout the tail, consistent with the comet tail-like draping picture (Fig. 5b). In the hemisphere where the solar wind convection electric field points towards the planet ( $-E$-hemisphere), however, a reversal of this component is observed (Fig. 13). In this hemisphere the field lines wrap tighter around the planet (Zhang et al. 2010) creating a reversal of the cross flow component appearing at the terminator and observable out to a few planetary radii in the tail (Du et al. 2013; Rong et al. 2014). When the field reversal appears at the terminator it is confined to a region close to the magnetic pole. Moving tailward, the region with a reversed cross flow component grows until it covers the entire $-E$-hemisphere and parts of the $+E$-hemisphere as 

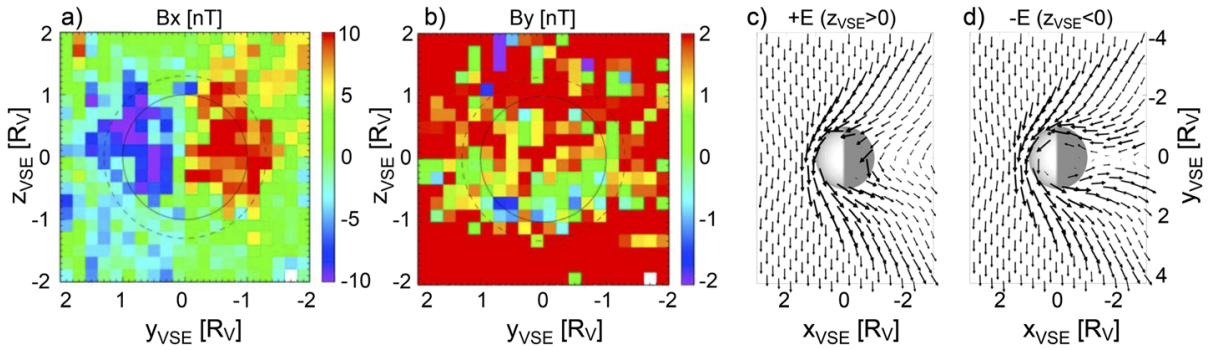

Fig. 13 The distribution of the cross-tail magnetic field $B_{x}$ and $B_{y}$ components seen by VEX/MAG instrument is shown to the left two panels. $B_{x}$ represents the typical lobe structure without hemispherical asymmetry, while $B_{y}$ shows strong asymmetry. Note the reversal of the cross flow component in the $-E$-hemisphere close to the planet. Results from a hybrid model (right two panels) show a similar asymmetry (after Zhang et al. 2010)

well (Du et al. 2013). Even further tailwards the reversed field region is again restricted to the $-E$-hemisphere (Rong et al. 2014).

The observed draping pattern, which is reproduced by a hybrid model, suggests the possibility of magnetic reconnection and the formation of an $X$-point in the $-E$-hemisphere (Zhang et al. 2010). Looking closer more differences are revealed between the hemispheres. The magnetic field is stronger in the $+E$-hemisphere. The difference is large in the magnetic barrier on the dayside, gets less prominent at the terminator, but can still be seen in the near tail (Du et al. 2013). These hemispheric asymmetries may be caused by pickup ions slowing down the magnetosheath flow in the $+E$ hemisphere. Many of the planetary ions that are picked up in the $-E$-hemisphere do not reach the magnetosheath but gyrate into the atmosphere (Luhmann et al. 2006). In addition, some models suggest that a pickup process may not be necessary to explain such an asymmetry if the Hall effect (i.e. different electron and ion behaviors) is considered (Brecht 1990; Brecht and Ferrante 1991).

The magnetic field component along the convection electric field direction is such that the magnetic field wraps the planetary body and the field lines close in on the magnetic equator from both hemispheres, or as expressed by Rong et al. (2014) the magnetic field "sinks into the Venus umbra".

In the now accepted picture of the magnetotail, the magnetic field lines are nicely structured in the $+E$-hemisphere, displaying a clear current sheet. Moving toward the magnetic equator the current sheet gets thinner (the cross flow component is less prominent), and in the $-E$-hemisphere the average pattern is more irregular. Fitting a Harris sheet model to the average current sheet profile between $-1.5 R_{\mathrm{V}}>x_{\mathrm{VSO}}>-2.5 R_{\mathrm{V}}$ gives a central current sheet half width of $460 \mathrm{~km}$ in the $+E$-hemisphere and $\sim 200 \mathrm{~km}$ in the central region $\left(\left|z_{\mathrm{VSE}}\right|<0.5 R_{\mathrm{V}}\right.$; Rong et al. 2014). There are, however, many different ways to define the width of a current sheet, which results in vastly different outcomes, so the most important result is that the current sheet is thinner close to the magnetic equator. The current density is similar though, and the larger magnetic cross flow component in the $+E$-hemisphere leads to a stronger tailward $\boldsymbol{j} \times \boldsymbol{B}$-force there (Rong et al. 2014).

The current sheet is not always well-described with a Harris sheet. Sometimes the sheet appears to have a double-scale structure and is better modeled by the sum of two Harris-type functions, i.e., $B_{1} \tanh \left(t / T_{1}\right)+B_{2} \tanh \left(t / T_{2}\right)$ with $T_{2}>T_{1}$ (Vasko et al. 2014), where $B_{1}$ and $B_{2}$ is the $B_{x}$ components, $t$ is the time and $T_{1}$ and $T_{2}$ are the typical temporal scales. The apparent double-scale nature seen in data may result from a multiple species ion population in the tail. The observed magnetic profile can be explained by a current sheet model, where 
protons are responsible for the inner scale and oxygen ions create the outer scale (Vasko et al. 2014). Observations from the Earth's magnetoshere suggest that the presence of oxygen ions in the tail during disturbed times similarly results in a double-scale profile of the current sheet (Zelenyi et al. 2011).

\subsection{Magnetic Reconnection in the Magnetotail}

Magnetic reconnection has been observed in the magnetospheres of Earth, Mercury, Jupiter and Saturn (e.g., Walker et al. 1999; Øieroset et al. 2001b; Slavin et al. 2009; Russell et al. 1998; Jackman et al. 2007) as well as in astrophysical plasmas (e.g., Shibata 1996; De Gouveia Dal Pino et al. 2010) and in the laboratory (e.g., Ren et al. 2005). It is also believed to cause the observed disconnection of comet tails (Niedner and Brandt 1978; Russell et al. 1986). Signatures of reconnection have also been reported from Mars (Eastwood et al. 2008; Halekas et al. 2009), specifically in the magnetotail current sheet. The observations at Mars include detection of the so-called Hall magnetic fields. Close to a reconnection $X$-line ions approaching the reconnection site decouple from the magnetic field, while the electrons continue to be frozen-in. The differential flow gives rise to currents and a quadrupolar magnetic field structure that can be observed in the diffusion region where opposing fields merge (e.g. Øieroset et al. 2001b).

Venus Express finally provided evidence that reconnection also occurs in the magnetotail of Venus (Zhang et al. 2012; Dubinin et al. 2012). Passing through the plasma sheet VEX observes spikes in electron and ion fluxes. Such bursts can be generated by different mechanisms but one suggestion is reconnection. Ions are seen moving alternately tailward and planetward, as the magnetic field changes in a way consistent with the spacecraft first entering and then leaving a magnetic island (Dubinin et al. 2012). A clear example of the same geometry in presented by Zhang et al. (2012). A well-defined flux rope is detected and at the same time enhanced ion and electron fluxes were observed (Fig. 14). The orientation of the flux rope is consistent with reconnection, as opposed to an earlier investigated case (Slavin et al. 2009). The plasmoid/magnetic island structure moved towards Venus and its size was estimated at up to $3400 \mathrm{~km}$ (Zhang et al. 2012). Hall magnetic fields, taken as evidence for collisionless reconnection in the Martian tail, have also been seen at the current sheet of Venus (Dubinin and Fraenz 2015). If reconnection occurs regularly in the Venusian tail it may be an additional mechanism contributing to the acceleration of plasma and atmospheric escape (see Sect. 9 below).

\subsection{Plasma in the Magnetotail}

A range of ion populations in the magnetotail of Venus was identified in the combined Venera and PVO observations (see Vaisberg et al. 1995 for an overview and references therein). It was already realized from those measurements that there were a number of different contributing source regions and mechanisms for the tail ion acceleration. In particular, these authors noted the existence of a cold heavy ion (mainly $\mathrm{O}^{+}$) population associated with both the post-terminator IMB and the current sheets in the wake, including the current sheets at the wake boundary shear layer as well as the induced, draped field tail current sheet. There was also the clear evidence of a convection electric field asymmetry and so reconciling these behaviors presented challenges.

Figure 15 from Barabash et al. (2007a), where the plasma data have been organized by the magnetic field assuming the upstream electric field $\left(\boldsymbol{E}=-\boldsymbol{V}_{S W} \times \boldsymbol{B}\right)$ determines the vertical axis (VSE, Venus-Solar-Electric, system), clearly shows these asymmetries in both 

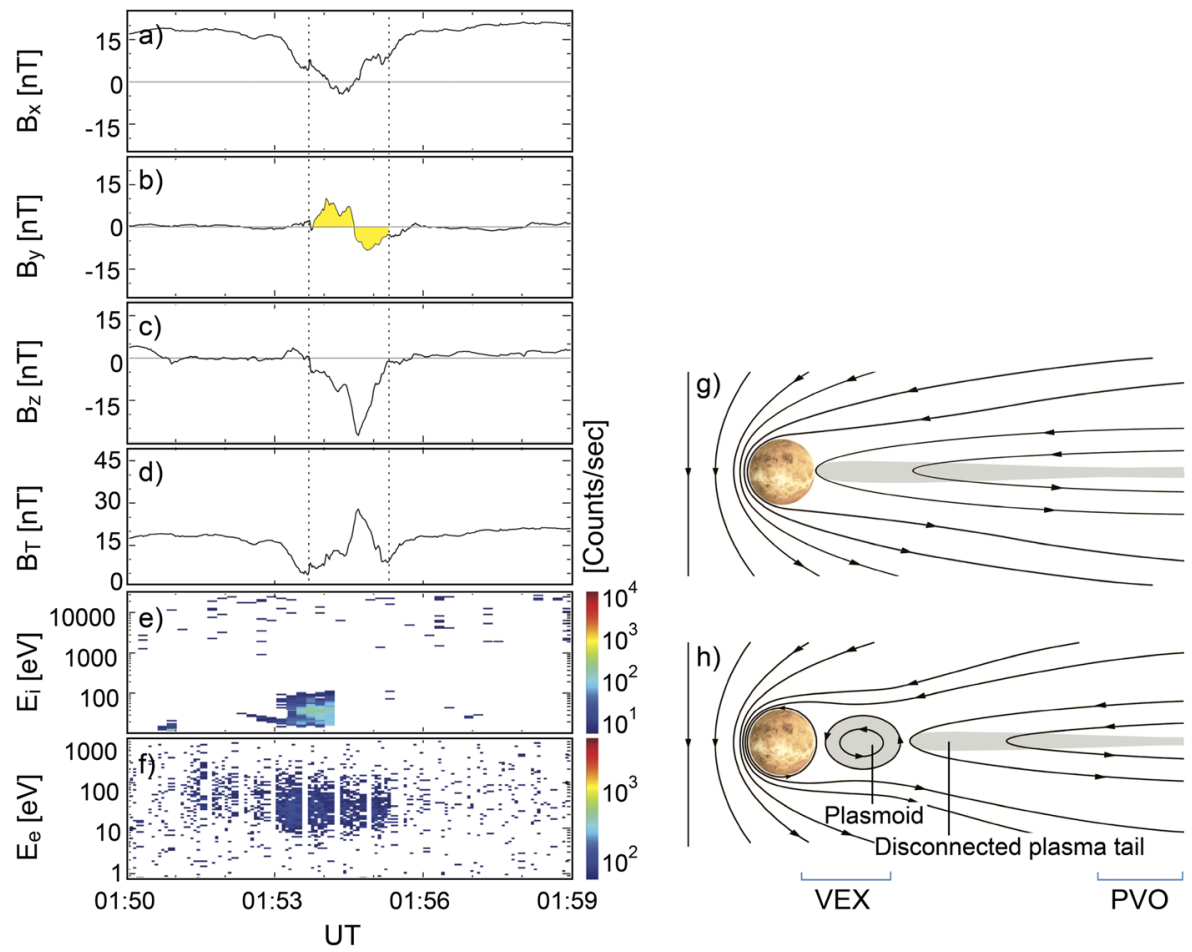

Fig. 14 Observation of a plasmoid flux rope in the Venusian magnetotail. The magnetic field components in panels (a-d) are rotated into a frame where the $x$-component is along the ambient field before and after the event. Ion (e) and electron (f) data are shown in the bottom two panels. The assumed geometry is sketched to the right $(\mathbf{g}, \mathbf{h})$. The observed orientation of the flux rope is consistent with magnetic reconnection (after Zhang et al. 2012)
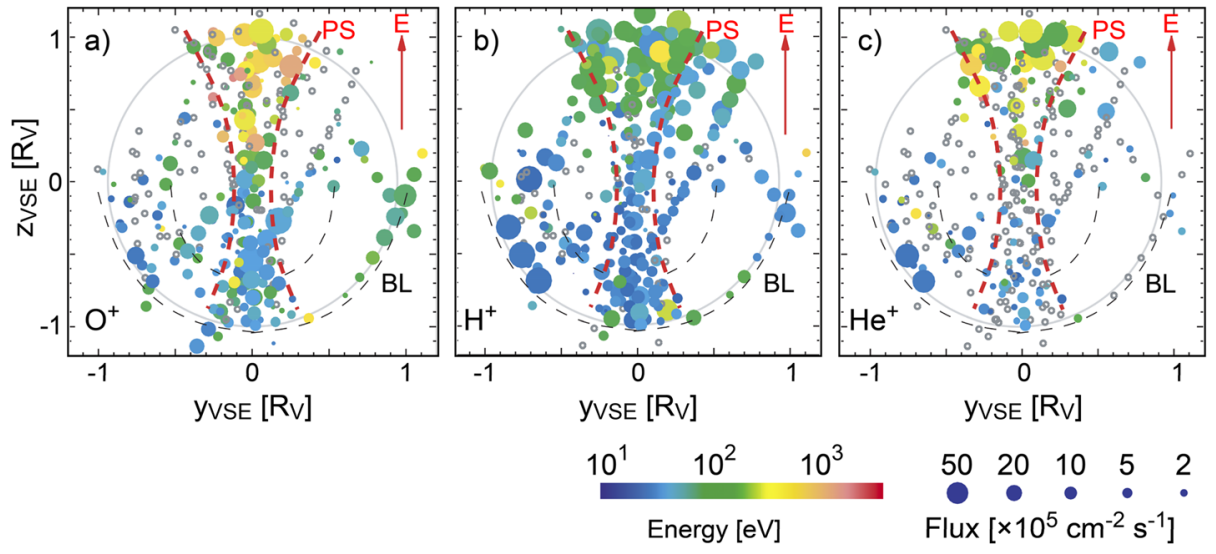

Fig. 15 An average cross section view of the ion fluxes in the Venusian tail in the VSE coordinate system. The convection electric field direction is along the $y$-axis. The observed outflowing ions, (a) $\mathrm{O}^{+},(\mathbf{b}) \mathrm{H}^{+}$and (c) $\mathrm{He}^{+}$from Venus are drawn. The color of the data points represents the ion energy, and the size of the points indicates the flux (adopted from Barabash et al. 2007a) 

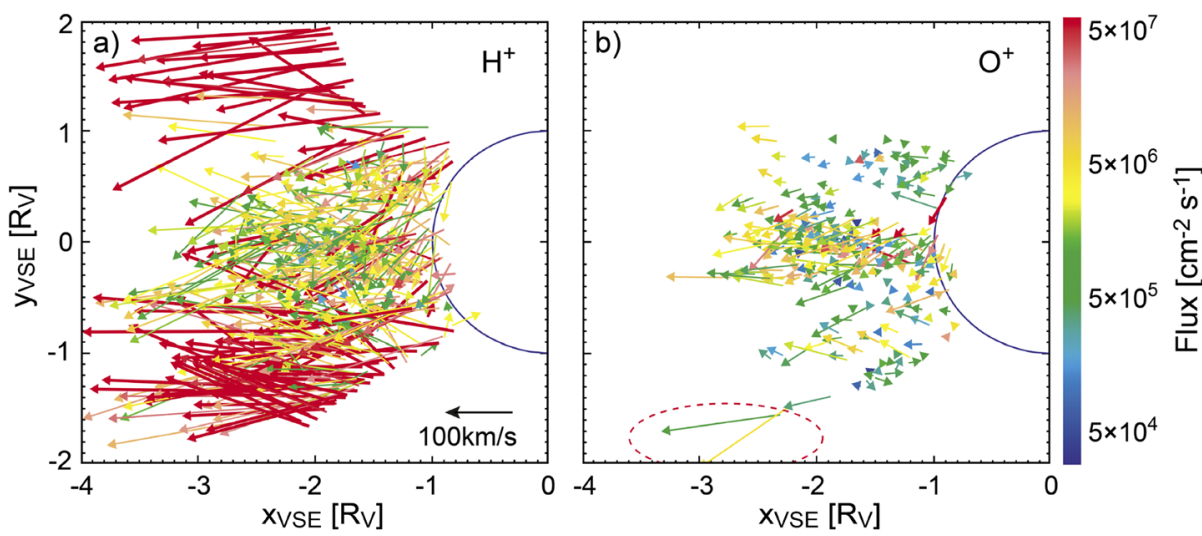

Fig. 16 The complex flows of plasma in the magnetotail, where both planetward and antisunward flows are observed. The color scale gives the integral flux while the length of each arrow corresponds to the velocity (adopted from Fedorov et al. 2011)

the heavy ion fluxes and energies in an average wake cross section view. The density of oxygen ions is lower in the $+E$-hemisphere but the bulk velocity is higher. Here the draped field pushes the plasma into the tail by means of the $\boldsymbol{j} \times \boldsymbol{B}$-force. In the $-E$-hemisphere the flow pattern is more irregular and a planetward motion is more often observed (Dubinin et al. $2013 \mathrm{~b}$ ). As discussed above the magnetic field is more tightly wrapped around the planet in this hemisphere and the average field pattern does not show a distinct current sheet but is more irregular (Zhang et al. 2010; Rong et al. 2014). The acceleration of the ionospheric plasma into the tail is further discussed later in connection with atmosphere escape (Sect. 9). VEX observations also reveal that most of the time there is no pressure balance between the magnetic field in the tail lobes and the thermal ion pressure in the plasma sheet. The ions stream as cold beams and it is instead the dynamic pressure that provides a balance. The pressure balance in the tail was discussed earlier based on PVO measurements (Saunders and Russell 1986) but the issue could not be resolved due to limitations of the data.

The plasma flows in the wake of Venus (Fig. 16) show a complex combination of sunward (planet-ward) and antisolar flows (Fedorov et al. 2011). Some of the observed irregularities may be effects of the reconnection described above. In addition, in a geographical frame of reference the plasma flow in the tail takes the shape of a large vortex. The vortex begins at the dusk flank with ions flowing dawnward/tailward. Solar wind protons and heavy ionospheric ions display the same vortex pattern, suggesting that energy and momentum are directly transferred from the solar wind plasma to the planetary ions. The origin of the vortex has been proposed to be the orbital motion of Venus, which causes a significant aberration of the solar wind flow around Venus (Lundin et al. 2013).

Combinations of these new observations in the near-Venus tail region on VEX, together with improved models (see also Sect. 11) that include more of the physics of the ion acceleration and spatial distributions (e.g. see Jarvinen et al. 2010, 2016) have made further progress in untangling the complicated planetary ion magnetotail of Venus.

\section{Wave Phenomena and Their Relationships}

The interaction between the solar wind the induced magnetosphere of Venus creates many types of fluctuations in the involved plasmas and fields. Some of them can be interpreted 
in terms of linear wave modes, others are non-linear in nature and some are best described as turbulence. In some cases, the waves seem to be locally generated, perhaps by unstable particle distribution functions, while in other cases they seem to have remote sources and have propagated to the site of observation. Waves can play important roles in modifying the particle distribution function. They may cause scattering and phase space diffusion as well as particle heating and acceleration to energies exceeding the escape energy. Waves can also be a useful diagnostic tool, for example, in studies of extended exospheres. We provide a brief overview of what has been observed in and around Venus' induced magnetosphere.

\subsection{Upstream Waves}

Far upstream of Venus there are already signs of an approaching planet. The hydrogen exosphere reaches beyond the bow shock and the appearance of newly ionized exospheric ions leads to the formation of an unstable ion population in the solar wind. Although the particle density is far too small to be detected, the waves that are generated can be observed, providing evidence of the removal of hydrogen from the atmosphere. From linear theory highest growth rate is expected for a circularly polarized mode propagating parallel to the ambient magnetic field (Gary 1991). Compared to the solar wind, newborn pickup ions have a negligible velocity relative to the planet and the observing spacecraft and the generated waves will therefore be observed at the spacecraft at the local proton gyro frequency (Delva et al. 2011). They are referred to as proton cyclotron waves (PCW).

Proton cyclotron waves are observed upstream of many objects in the solar system, including Mars, comet 1P/Halley and the Jovian satellites Io and Europa (e.g., Russell et al. 1990; Huddleston et al. 1997; Volwerk et al. 2001; Johnstone et al. 1987). They were expected also at Venus but in PVO data they were only seen in the magnetosheath and never upstream of the bow shock. Even a newly made re-examination of PVO data (Russell et al. 2006a) did not reveal upstream waves with frequencies close to the proton gyro frequency. The discovery of upstream proton cyclotron waves at Venus (Delva et al. 2008a) occurred with the arrival of VEX.

VEX observations of proton cyclotron waves resemble measurements made at other solar system bodies. The waves are circularly polarized, left-handed in the spacecraft frame and the wave power peaks just below the local proton gyro frequency. They propagate with a small angle to the ambient magnetic field, either almost parallel or almost anti-parallel. The waves are encountered in a large region upstream of the bow shock, up to $9 R_{\mathrm{V}}$ from the Sun-Venus line and up to $4 R_{\mathrm{V}}$ in the sunward direction (Delva et al. 2008b). They are recorded both within the foreshock and outside, supporting the idea that their generation is indeed due to exospheric pickup ions and not due to ion interaction with the shock itself. The locations of the wave observations are seen in Fig. 17.

The solar wind electric field does not play a role for the probability of detecting proton cyclotron waves, which is an indication that the waves are generated from the ion distribution function resulting from the initial pickup. Ions that are picked up can neutralize again in a charge-exchange process and travel far from the planet as fast neutrals before they again are ionized. The re-neutralization is likely to happen during the first part of the first gyration, which means that the fast neutrals all travel in the direction of the solar wind electric field. Hence, if secondary ionization of pickup ions would be responsible for proton cyclotron waves at Venus one would expect a non-measured asymmetry with respect to the electric field (Delva et al. 2008b, 2011).

An important parameter for generation of proton cyclotron waves is the angle between the solar wind velocity and the interplanetary magnetic field. For a perpendicular configuration 
Fig. 17 Spatial distribution of the proton cyclotron wave observations. Largest amplitudes (color scale) are observed close to the bow shock where the relative density of pickup ions is high. Most cases are also found downstream of the terminator where the waves have had more time to grow enough to be detected. Black circles denote observations made upstream of the foreshock region and the lines show approximately the limiting orbits of the spacecraft (after Delva et al. 2008b)

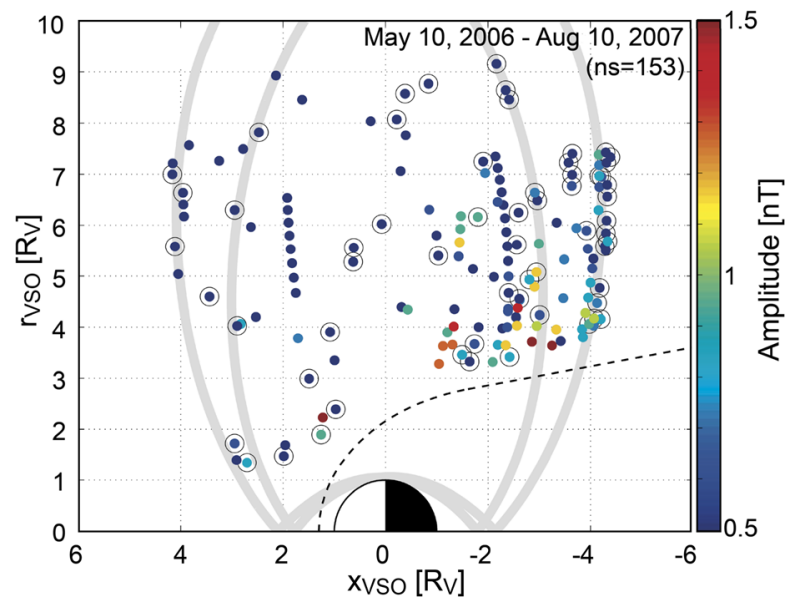

the pickup ions form a (pure) ring distribution in velocity space, while for the more parallel (or antiparallel) case the result is a beam distribution. If the perpendicular velocity component is small, most of the free energy is in the drift motion of the ions and ion-ion beam instability is responsible for fast growth of cyclotron waves (Gary 1991). The waves generated through the ion-ion beam instability are intrinsically right-handed in the plasma reference frame, but the phase velocity is small compared to the solar wind velocity. Hence, they are observed as left-handed waves in the spacecraft frame (Mazelle and Neubauer 1993).

Parallel or antiparallel magnetic field configurations indeed seem to favor generation of proton cyclotron wave at Venus (Delva et al. 2011). The conditions also need to remain stable for a certain time in order for the waves to grow. Most observations last ten minutes or less and they are seen during $1 \%$ of the time (Delva et al. 2008b). The stable solar wind conditions required for efficient wave growth are not often met and especially not over longer times spans (Delva et al. 2011). The occurrence increases during solar maximum even though stable solar wind conditions are even more rare then. The key parameter turns out to be the relative density of the pickup ions and the background solar wind (Delva et al. 2015). The EUV flux is higher leading to an increased ionization of the exosphere/corona and at the same time the solar wind density is lower, especially during Solar Cycle 24, when the measurements were made. We note that an independent study of ICWs at Venus using VEX data and different software for identifying ICWs did not detect any ICWs at Venus (Wei et al. 2011).

Another source of upstream waves is ions being reflected at the bow shock. They give rise to ultra low frequency (ULF) large amplitude waves, which attempt to propagate upstream but are convected back towards the bow shock. Such waves are believed to steepen and evolve into SLAMS (Short Large-Amplitude Magnetic Structures, see Sect. 5) when they interact with diffuse ions on their way back to the shock (Schwartz et al. 1992). However, Shan et al. (2014) presents a case study using VEX magnetometer data, where monochromatic ULF waves are seen to pass through the bow shock, from the upstream to the downstream side. The idea of waves passing the bow shock has been proposed before (Luhmann et al. 1983) but the process had not been observed directly. The waves are identified as magnetosonic waves, with frequencies well below the local proton gyro frequency. They are transverse, left-hand polarized in the spacecraft frame and the angle between the propagation direction and the ambient magnetic field is $40-60^{\circ}$. The waves keep their properties during the transmission. Just a few similar cases were found in VEX data, all for quasi-parallel 
shock conditions. The small size of the Venusian foreshock may be the reason why, in these cases, the ULF waves did not grow into SLAMS.

Upstream waves also include mirror mode waves. In the solar wind they manifest themselves as isolated intervals with a depressed magnetic field strength, with a typical duration of seconds to 10s of seconds (Turner et al. 1977). The magnetic field direction does not change across the magnetic depression, which is also called a linear magnetic hole. Zhang et al. (2008e) determined the shape of mirror mode structures in the solar wind at a heliocentric distance of $0.72 \mathrm{AU}$ (Venus orbit). They found that a mirror mode structure is best described as a rotational ellipsoid elongated along the magnetic field. The width across the magnetic field (minor axis) is typically 42 times the gyro radius of a solar wind proton. The ratio between the major and minor axis of the ellipsoid is 2.55 .

\subsection{Magnetosheath, Mantle and Tail}

Mirror mode waves are found not only in the solar wind at different distances from the Sun, but also in the magnetosheaths of Earth (Tsurutani et al. 1982), Mars (Bertucci et al. 2004) and Jupiter (Erdös and Balogh 1993). Mirror mode waves are compressional, may have large amplitudes and propagate almost perpendicular to the ambient magnetic field. Generation of mirror modes requires a high- $\beta$ plasma and a temperature anisotropy with $T_{\perp} / T_{\|}>1$. The temperature anisotropy can be created by ions reflecting multiple times at the bow shock before entering the magnetosheath or by a compression of the magnetosheath close to the magnetopause. Mirror mode waves are therefore observed more frequently downstream a quasi-perpendicular bow shock, where a temperature anisotropy can build up. Mirror mode waves are, however, sometimes seen even under quasi-parallel conditions (Volwerk et al. 2008b). However, in a study using PVO data obtained in the subsolar magnetosheath no clear evidence of mirror mode waves were found (Luhmann 1995). The first indication of the existence of mirror mode waves is reported by Volwerk et al. (2008a) based on magnetic field data from VEX. The mirror mode waves are seen in two regions of the magnetosheath: just behind a quasi-perpendicular bow shock and close to the magnetopause. They are most likely to be observed on the dayside but occur quite frequently also in the wake, usually though with lower amplitudes (Volwerk et al. 2008b). The wave period observed at Venus is 5-15 seconds, which is shorter than for the corresponding waves seen in the Earth's magnetosheath (Volwerk et al. 2008a).

As discussed in the previous subsection proton cyclotron waves were never seen in PVO data upstream of the Venusian bow shock. They were, however, observed within the magnetosheath with all the characteristic properties of proton cyclotron waves: They have a frequency close to the local proton gyrofrequency, are transverse and left-hand polarized in the spacecraft frame and propagate with a small angle with respect to the ambient magnetic field (Russell et al. 2006a).

Downstream of the bow shock, nearly circularly polarized, non-linear, large-amplitude waves are occasionally observed (Walker et al. 2011). They are assumed to be waves standing with respect to the ionosphere and have sizes of 100-300 km. Modelling (Terada et al. 2002; Amerstorfer et al. 2007; Biernat et al. 2007) predicts that such waves should exist in Venus magnetosheath in association with Kelvin-Helmholtz instabilities. The observed wave structure was successfully reproduced with a one-dimensional two-fluid hydrodynamics model (Golbraikh et al. 2013). It cannot be completely ruled out that the generation of these nonlinear large-amplitude waves are tied to the bow shock, as Luhmann et al. (1986) proposed when investigating the Earth magnetosheath. For example, Balikhin et al. (2008) observed magnetic oscillations close to the Venusian bow shock that appeared to be part 
Fig. 18 Considering the statistical properties of magnetic field turbulence, the fluctuations can be characterized by their spectral indices. The scaling properties of magnetic fluctuations are found to be different in different regions of the Venusian magnetosphere reflecting the dominating wave types in each region (adopted from Vörös et al. 2008b)

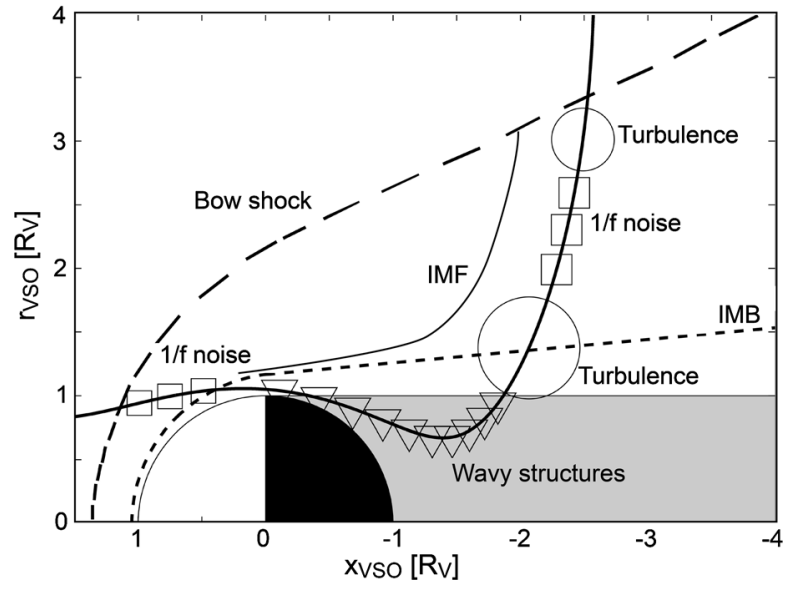

of the structure of a low Mach number, low- $\beta$ shock (cf. Sect. 5) but linearly polarized as opposed to the waves reported by Pope et al. (2009) and Walker et al. (2011).

Zhang et al. (2007a) noted that different plasma regions are clearly identifiable from the strength and variation of the wave or fluctuation activity in the magnetic field. Hence, an alternative way to look at waves around Venus is to focus on their statistical properties. The fluctuations can be characterized in terms of their spectral indices and the scaling properties of the fluctuations appear to be different in different regions (Vörös et al. 2008a, 2008b). In the magnetosheath on the dayside, and far away from any plasma boundaries on the nightside, the spectral scaling index, $\alpha$, is found to be close to one. The wave power then scales as $\sim 1 / f^{\alpha} \sim 1 / f$, where $f$ is the frequency. Guicking et al. (2010), however, got a different statistical scaling index using more data and estimated $\alpha=1.8-1.9$ in these regions for a similar frequency range, that is, below $\sim 0.5 \mathrm{~Hz}$. Near the terminator and in the wake the spectral index $\alpha=2.4-2.5$. It suggests the coherent wavy fluctuations found here are likely associated with a Kelvin-Helmholtz instability and the non-linear large amplitude waves mentioned before is probably one possible manifestation. Figure 18 shows a map of the different scaling indices found in the induced magnetosphere.

Close to the bow shock and the upper mantle boundary spectral indices close to what would be expected for hydrodynamic or MHD turbulence are observed. The occurrence of intermittency further supports the interpretation with the implication of related dissipation and plasma heating (Vörös et al. 2008b). Statistically, the wave intensity of low frequency waves $(30-300 \mathrm{mHz})$ is highest in the dayside magnetosheath and decreases rapidly at the terminator. The compressional and transverse components of the wave power are equal and the sense of polarization varies. The average wave vector and the average local mean magnetic field are almost perpendicular at low altitudes but angle between them decreases closer to the bow shock. In the nightside magnetosheath the intensity is lower, the waves are more transverse and the angle between the wave vector and the magnetic field typically less than $45^{\circ}$ except close to the bow shock (Guicking et al. 2010). The high wave intensity close to the bow shock suggests that most waves are generated there and subsequently convected downstream, but wave generation also takes place in the magnetosheath itself. The rapid drop in wave intensity at the terminator remains to be explained. In the mantle and tail regions the ellipticity is small and the waves appear linearly polarized (Du et al. 2010).

The orientation of the interplanetary magnetic field has a strong effect on the lowfrequency wave properties in the near Venus region (Du et al. 2009). The relative total power 
(total wave power divided by $|B|^{2}$, where $B$ is the background magnetic field) in the magnetosheath is considerably higher when the cone angle, the angle between the interplanetary magnetic field and the incident solar wind velocity vector, is small. The intensity decreases with solar zenith angles, which again suggests that the bow shock area is the main wave generation region. As previously discussed, during quasi-parallel conditions waves generated in the foreshock can be convected into the magnetosheath. The fluctuations tend to get more transverse for a large cone angle. They are also left-handed and elliptically polarized, and may comprise the proton cyclotron waves described above (Du et al. 2010).

\subsection{Ionospheric Sources}

Lightning has long been proposed to take place in the dry but cloudy atmosphere of Venus. Both optical and electromagnetic observations supporting this have been reported (Taylor et al. 1979; Krasnopolsky 1983). As on Earth the discharges are expected to create electromagnetic whistler mode waves propagating from the atmosphere into the ionosphere. Measurements consistent with whistler mode waves were observed using the electric antenna on PVO. Other attempts to demonstrate the occurrence of lightning failed, however, and no final conclusion could be agreed on. This motivated a special mode to be designed for the magnetometer onboard VEX in order to detect whistler waves originating from lightning (Russell et al. 2006b, 2007).

The effort paid off. Nearly circularly polarized waves propagating almost parallel to the background magnetic field were detected using a frequency window of $42-60 \mathrm{~Hz}$ (Russell et al. 2007). The wave emissions were bursty with a typical burst lasting up to half a second (Russell et al. 2008a). The probability of detecting a whistler mode wave emission at a particular time depends on the occurrence of discharges. Assuming observations made around the periapsis of VEX (above the north pole) are representative for the entire planet, the estimated global rate is 18 strokes/s, which is $20 \%$ of the terrestrial value. For the waves to be detected they must also be able to reach the spacecraft. Whistler mode waves do not propagate perpendicular to the ambient field and the average orientation of the local ionosphere magnetic field at periapsis is horizontal. Russell et al. (2008b) showed that the magnetic field has to be inclined $17^{\circ}$ or more to allow whistler emissions to reach the spacecraft.

Lightning related whistler mode waves occur at all local times but are most frequently observed at local times close to the terminator (Daniels et al. 2012). The amplitude and occurrence of the waves depends on altitude and both burst rate and amplitudes are small below $200 \mathrm{~km}$. A possible explanation is that the lower electron density causes the waves to propagate faster and hence the amplitude decreases to keep the energy flux constant. This hypothesis is also consistent with a slowly decreasing occurrence rate above $225-250 \mathrm{~km}$.

When an improved cleaning technique for VEX magnetic field data made a wider bandwidth available another type of electromagnetic wave was seen together with the whistler mode waves (Russell et al. 2013a). The new waves are often seen at lower frequencies (below $20 \mathrm{~Hz}$ ) compared to the whistlers, are linearly polarized and propagate at large angles to the magnetic field (see Fig. 19). The source of these perpendicularly propagating waves is unlikely to be lightning. Instead, they are probably generated at higher altitudes close to the ionopause (Russell et al. 2013b).

Similar waves were reported by Wei et al. (2012a) but were shown to be associated with thin current sheets in the ionopause region. These waves appear to be lower hybrid waves and may to contribute to current dissipation and heating of the plasma there. Indeed Strangeway and Russell (1996) had previously proposed an ionopause current-related instability to explain the plasma waves routinely observed in the Venus ionopause on PVO. These waves, 


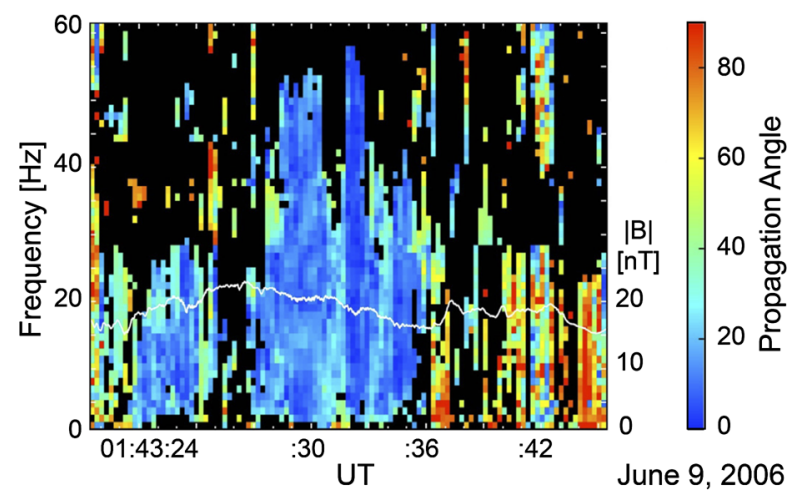

Fig. 19 Propagation directions of the two types of electromagnetic waves observed in Venus ionosphere. Until 0143:36 UT the spectrum is dominated by right-handed circularly polarized waves and propagating along the ambient field (whistler mode waves). Later linearly polarized waves, which may propagate across the magnetic field are seen. For the linear waves the propagation direction is indeterminate, which results in the mottled appearance. The white line shows the ambient magnetic field. Signals where the coherence is less than 0.5 are masked out (represented as black areas) (after Russell et al. 2013b)

though often neglected in analyses of pressure balance at the subsolar ionopause, may in fact play a role by altering the electron temperatures there. A high altitude source of ionospheric electron heating is in fact indicated by the Langmuir probe observations.

\section{Impacts on the Atmosphere}

Although both ionized and neutral gases can escape from all planetary atmospheres to some degree, in the case of Venus' induced magnetosphere solar wind interaction the related energization of the ionospheric ions is nominally the most important process for species heavier than hydrogen. The upper atmospheric atoms (mainly $\mathrm{H}$ and $\mathrm{O}$ ) are ionized by the solar EUV, charge exchange, and solar wind electron impact ionization. If the acceleration gives those particles more than the escape velocity, the particles can be lost from the planet. The escape velocity for Venus is $10.4 \mathrm{~km} / \mathrm{s}$, corresponding to $8.7 \mathrm{eV}$ for $\mathrm{O}^{+}$. This is higher than the escape velocity at Mars $(1.9 \mathrm{eV})$, where even photochemical processes are important for energizing neutral atoms to escape speeds. The escape energy for oxygen at Venus of $8.7 \mathrm{eV}\left(\sim 10^{5} \mathrm{~K}\right)$ is too high to reach by thermal spread or photochemical processes. This means that Jeans escape is minor for all major atmosphere constituents at Venus, and that the ionospheric dissociative recombination process $\left(\mathrm{O}_{2}^{+}+\mathrm{e}^{-} \rightarrow \mathrm{O}^{*}+\mathrm{O}^{*}\right)$, that is so effective at Mars, cannot produce atomic oxygen with more than the escape energy at Venus (see review by Dubinin et al. 2011). Therefore, the nonthermal escape driven by the solar wind is the most important mechanism for Venus atmosphere loss to space.

As reviewed in earlier sections, the interaction inside the induced magnetosphere and magnetotail can accelerate ionized particles significantly. Forces acting on these particles are mainly the convection electric field (leading to ion pick up), magnetic tension force (or $\boldsymbol{j} \times \boldsymbol{B}$ force), and the polarization electric field (parallel electric field). These three forces are effective in the exosphere, the upper thermosphere above the collisional regions (inside the IMB), and in the wake and plasmasheet (Fig. 15). In addition, ionospheric plasma may sometimes be shed from the ionopause region as dense "plasma clouds" due to boundary 
Fig. 20 A summary of the total escape rate as a function of the energy range (figure updated from Dubinin et al. 2011, with more recent reports being added). Estimated escape rates vary depending on data used, data analysis methods and energy range considered. From the most of the reported ASPERA-4 observations, here we can conclude that the total outflowing flux is $(3-6) \times 10^{24} / \mathrm{s}$ in the solar minimum. See discussion in text also

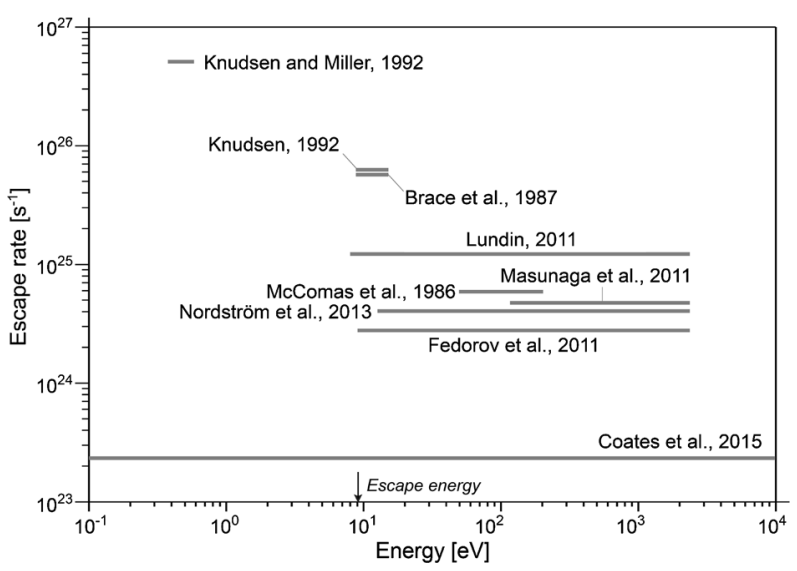

layer instabilities and/or transient reconfigurations of the magnetic field. Wave particle interactions may also cause additional acceleration of planetary ions.

Isotopic evidence on the $\mathrm{D} / \mathrm{H}$ ratio from the Pioneer Venus probe, as well as remote sensing measurements of the Venus' hydrogen corona have suggested that there has been significant escape of hydrogen at Venus compared to the Earth over time (Donahue et al. 1982). Other isotope analyses and atmosphere composition studies support this idea, with interesting implications for the now scarce water content of the atmosphere. As already mentioned above, solar wind interaction-related heavy ion escape, including $\mathrm{O}^{+}$, may have been a key part of the history of a Venus ocean. Thus the amount of the escaping flux today, and its implications for past escape, is essential to determine. McComas et al. (1986) used the pressure balance assumption and inferred $\boldsymbol{j} \times \boldsymbol{B}$ force in PVO distant magnetotail observations to estimate the total plasma density and velocity in the plasma sheet and the associated escape rate $6 \times 10^{24} \mathrm{~s}^{-1}$. Brace et al. (1987) used PVO Langmuir probe measurements of the ionospheric density near the terminator, together with assumptions regarding the plasma velocity, to obtain the escape rate $5 \times 10^{25} \mathrm{~s}^{-1}$-although the escape cross section was not fully sampled, but assumed. This contrasted with the more direct measurements on PVO of the low energy ionospheric ion fluxes, which indicated a nightward trans-terminator flow of cold $\mathrm{O}^{+}$of $5 \times 10^{26} \mathrm{~s}^{-1}$ (Knudsen and Miller 1992). One of the ambiguities of these low altitude measurements is how much actually escapes as opposed to supplying the observed nightside (bound) ionosphere.

The latest plasma ion spectrometer measurements by the ASPERA-4 IMA provided a significant improvement on these estimates because they were obtained over a much more complete energy range, at altitudes above the most gravitationally bound ionospheric plasma, and with ion composition information (Barabash et al. 2007b). Using the dataset from the declining phase of the solar activity (2006-2007), the total escape rate covering all ion acceleration processes in the energy range $10 \mathrm{eV} / \mathrm{q}-25 \mathrm{keV} / \mathrm{q}$ was found to be $3 \times 10^{24} \mathrm{~s}^{-1}\left(\mathrm{O}^{+}\right)$. The VSE coordinate system was used for this analysis (Fedorov et al. 2011). Analysis of a more extended data set (2006-2009), using both VSE and orbit based systems gave the $\mathrm{O}^{+}$escape rate of (4.3-5.2) $\times 10^{24} \mathrm{~s}^{-1}$ (Nordström et al. 2013). Lundin (2011) used the orbit coordinate system (2006-2009), and shifted the energy table to lower energies (to account for spacecraft potential) to derive an $\mathrm{O}^{+}$escape rate of $1.2 \times 10^{25} \mathrm{~s}^{-1}$. For typical conditions, Masunaga et al. (2011) derived a rate of $(5.8 \pm 2.9) \times 10^{24} \mathrm{~s}^{-1}$ for $\mathrm{O}^{+}$with energy $>100 \mathrm{eV}$. From photoelectron measurement on the field connected to the ionosphere, Coates et al. (2015) estimates the total ion outflow to be $2.2 \times 10^{23} \mathrm{~s}^{-1}$. The estimate is 
lower than the other values derived from direct ion measurements, but this value is derived only from a single observation. Each of these inferred escape rates are based on different data corrections, assumptions regarding energy and angle coverage, and extrapolations of orbit-sampled data to a global picture.

It is important to recognize the difficulties of calculating the total escape rates from the observations. Frequently, the total escape rates are different, even though researchers used the same dataset. The rate is very sensitive to the assumptions used for the calculation.

The choice of the coordinate system may matter, particularly when the dataset size is small. Many authors convert the coordinate system into the VSE frame mentioned in the earlier discussions (e.g. Barabash et al. 2007a; Fedorov et al. 2011). The weakness of the VSE frame is that the frame can only be well-defined when the IMF is fairly steady. This may result in the bias of the observation, namely, deriving the outflow flux under calm conditions. It could underestimate the outflow flux considering the potential increase of the outflow during the variable conditions (Futaana et al. 2008; Edberg et al. 2011). Other authors use the orbital VSO (Venus-Solar-Orbit) frame simply referenced to the Sun and the ecliptic plane (e.g. Lundin et al. 2011). Use of VSO may be suitable to discuss geometric influences (e.g. rotation of Venus, aberration of the magnetotail by Venus orbital motion). However, the weakness of the VSO frame is that the measurement region is highly biased due to the spacecraft orbit. Recently, Nordström et al. (2013) pointed out that the choice of the reference frame does not seem to be a big issue, in particular for the case of a large volume dataset, finding the relative difference of the total escape flux between VSO and VSE system is $<10 \%$.

Another difficulty of calculating the flux is due to the fact that it involves both spatial sampling and energy and angle coverage. The integration area assumed for the global escape extrapolation is another issue; correctly calculated escape rates must not depend on an assumed escape area, or the particular instruments used.

Moreover, it is a particular challenge to calculate the total escape rate at low energies driven by the solar wind. Due to the large contribution to the total flux, the accurate measurement and proper interpretation and data processing of low energy ion data are essential to calculate the total escape flux. The escape energies are close to the typical spacecraft potential (minus a few $\mathrm{eV}$ in the Venus ionosphere) and the apparent energies introduced by spacecraft motion. Typically, the ion fluxes with energies up to twice of the spacecraft potentials are affected due to the distortion of their trajectories on approach to the spacecraft. In addition, low energy ion detection may depend on the instrument field of view with respect to the ram direction. Thus, only under certain conditions can we reconstruct the true low energy spectra and fluxes of the ions.

Although for all these reasons there is no universal way of calculating the correct escape flux from the obtained dataset, as seen in Fig. 20 all available published fluxes show rather consistent values, depending on the lower bound of the energy. A good number to use, based on the VEX mission results, for the escape rate during the solar minimum is $(3-6) \times 10^{24} \mathrm{~s}^{-1}$ for $\mathrm{O}^{+}$.

The ultimate goal of measuring ion escape from Venus is to estimate its impact on planetary atmosphere evolution. To correctly extrapolate backwards in time, the dependence on the external conditions must be established. In particular, the dependency on the solar cycle should be assessed precisely. A good example is seen in the parallel studies that have been employed for the Martian upper atmosphere and its erosion. For the solar minimum, the Mars Express/ASPERA-3 data indicated a heavy ion escape flux of $\sim 2 \times 10^{24} \mathrm{~s}^{-1}$ (Nilsson et al. 2011). Applying the same method to all the available Phobos-2 data, the flux of $(2-3) \times 10^{25} \mathrm{~s}^{-1}$ was obtained (Ramstad et al. 2013). This is almost the same as the estimates using four elliptical orbits by Lundin et al. (1990). The experience of Mars indicate 
that the Venusian plasma outflow should also depend on the solar activity because of the similar interaction. In addition, VEX measurements under the disturbed solar wind (which could be a proxy of the high solar activity) show higher outflow flux (Sect. 10). On the other hand, comparison with Pioneer Venus (observed during the solar maximum) inferred escape rates of $\mathrm{O}^{+}$implies that the escape rate at solar minimum does not vary much with solar conditions (McComas et al. 1986). However, the comparison is not so straightforward because the dataset, instruments, and the methodology used for calculating the escape rates have been different. Thus, the long term ion escape study for Venus remains, using the same method but different instruments, is to be done. The last, but not least aspect of interest in planetary atmospheric evolution studies is the chemical composition of the escaping constituents. As mentioned earlier, the amount of water vapor $\left(\mathrm{H}_{2} \mathrm{O}\right)$ is significantly less in the atmosphere of Venus than for the Earth (McBride and Gilmour 2004). VEX IMA also detects escaping planetary $\mathrm{H}^{+}$. By using a dataset obtained between May 18 and December 30 in 2006, Barabash et al. (2007a) calculated the relative escaping ion composition of $\mathrm{H}^{+}$and $\mathrm{O}^{+}$, deriving a ratio of 1.9. This is interpreted as indicating that $\mathrm{H}_{2} \mathrm{O}$ molecules are effectively escaping from Venus, avoiding imbalances that may produce an oxygen budget issue-and their related surface oxidation requirements-in the atmosphere. It is thus notable that VEX results show water molecules are still escaping from Venus today, as a result of the solar wind interaction.

\section{Effects of Extreme Conditions}

\subsection{Interaction at Extreme Solar Wind Conditions}

The morphology of the interaction between Venus and the solar wind strongly depends on the upstream conditions. For example, because of the pressure balance between the solar wind and the Venusian ionosphere, the solar wind dynamic pressure directly influences the shape of the induced magnetosphere. For the same reason, EUV flux can control the interaction via the increase of the ionospheric pressure due to the increase of the atmospheric ionization. Moreover, passing structures in interplanetary space, resulting from the solar wind stream structure or activity on the Sun (including coronal holes and coronal mass ejections), are causes of sometimes extreme variations in the solar wind interaction. Of particular importance are the solar wind changes associated with CIRs (corotating interaction regions) and ICMEs (interplanetary coronal mass ejections). The CIRs can produce significantly enhanced solar wind densities, dynamic pressures, and magnetic fields because they involve spiral-shaped compression ridges between low and high speed streams, and can also have bracketing shocks. The ICMEs however produce the strongest disturbances in solar wind parameters, with the largest events led by a shock and period of high density, shocked solar wind, followed by a $\sim$ day long period of large magnitude, rotating magnetic field (with normal to low densities) - the ICME 'driver' which sometimes can be described as a flux rope of coronal origin with tenths of AU diameter. While normal solar wind travels at $\sim 600 \mathrm{~km} / \mathrm{s}$ in its high speed streams (relative to the $\sim 350 \mathrm{~km} / \mathrm{s}$ low speed wind), ICME plasmas and fields can pass at speeds up to several thousand $\mathrm{km} / \mathrm{s}$. Both CIRs and ICMEs also accelerate solar energetic particles (SEPs), whose effects on the atmosphere of Venus are unknown. Both CIRs and ICMEs can also be followed by unusually rarefied regions, which have not had time to refill following the high speed disturbance. It is important to consider how the solar wind interaction reacts to these extreme conditions as well as to the average conditions. 


\subsection{Interaction with Disturbed Solar Wind}

Following the orbit insertion of Venus Express, several disturbed solar wind periods (ICME, CIR, and resulting SEPs) were reported (Futaana et al. 2008; Rouillard et al. 2009; Dorrian et al. 2010; Williams et al. 2011; Möstl et al. 2011; Feng et al. 2012). These periods are particularly important from the perspective of atmospheric evolution, because they may increase the atmospheric escape from unmagnetized bodies beyond that from nominal conditions (e.g. Luhmann et al. 2006, 2007; Futaana et al. 2008). Moreover, considering that solar activity and the related frequency of ICME passage is thought to have been greater in the early stage of the Sun's lifetime, the solar wind interaction under disturbed conditions could be a key to estimating the total amount of the atmospheric loss from Venus over its $\sim 4$ billion year post-impact history.

Only a few events impacted Venus during the first few years of the VEX mission. The orbit insertion in 2006 was at the end of the declining phase of cycle 23, and solar activity was low for several years after. McEnulty et al. (2010) surveyed the occurrence of ICMEs seen on VEX in 2006 and 2007, and identified 17 events. They reported that the highest energy pickup ions were accelerated to higher energies than usual closer to Venus, but no clear increase (or decrease) of the pickup ion flux was found, likely because the involved ICMEs were relatively weak. In 2010, the solar activity started to increase, and reached to the cycle 24 maximum in 2013-2014. The solar maximum in this period was still not very high (approximately half the sunspot number compared to the previous solar maxima; Fig. 2). However as shown from the VEX ICME survey undertaken by Good and Forsyth (2016), there were a few stronger events that may yield further insights on related effects on Venus ion escape rates.

A statistical approach was taken in a study by Edberg et al. (2011), where the authors selected $147 \mathrm{CIR/ICME}$ events to make a flux map of the outflowing heavy ions around Venus. Their calculated total escape rates during the stormy periods was increased from the nominal period by a factor of 1.9 (Fig. 21). The defined disturbed period in their study was $35 \%$ of total, during which approximately $50 \%$ of the outflowing heavy ion fluxes were observed. Locally at the same position, the increase of the fluxes during the disturbed periods sometimes exceeded over 100 times. This is consistent with the low-energy $\mathrm{O}^{+}$escape from Venus measured on PVO with the suprathermal ion mode of the neutral mass spectrometer during periods of enhanced solar wind conditions (Luhmann et al. 2007). The increased dynamic pressure or the related rotating IMF direction are suggested as candidate processes inside the Venus induced magnetosphere increasing the heavy ion outflow. Nordström et al. (2013) calculated the total escape rate depending on the stability of the magnetic field direction. For the stable ( $<45^{\circ} \mathrm{IMF}$ rotation between in- and outbound) condition, the escape rate was $4.0 \times 10^{24} \mathrm{~s}^{-1}$, and for unsteady ( $>45^{\circ}$ rotations) conditions it was $5.9 \times 10^{24} \mathrm{~s}^{-1}$. A rotating IMF direction can create a favorable configuration of the draped magnetic field for reconnection in either the dayside magnetic pile-up region or in the induced magnetotail (Edberg et al. 2011). In addition, the ionospheric plasma clouds observed on PVO are frequently seen when IMF is rotating (Ong et al. 1991). Thus unsteady IMFs, some of which are associated with CIR and ICME passages as well, can also increase the escaping planetary ion flux.

It is important to reiterate here that the evaluation of extreme conditions responses on VEX was limited by the conditions accompanying the long solar minimum of cycle 23 and the weak solar maximum of cycle 24. In particular, the ICMEs during the latter period did not provide the ranges of parameters observed during the PVO mission. Nevertheless, the observed responses can be used to evaluate models of the more extreme interactions. Solar 

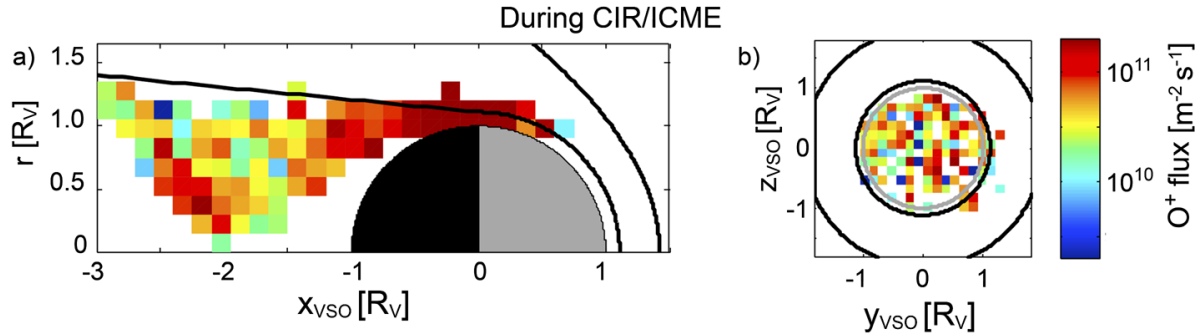

Not during CIR/ICME
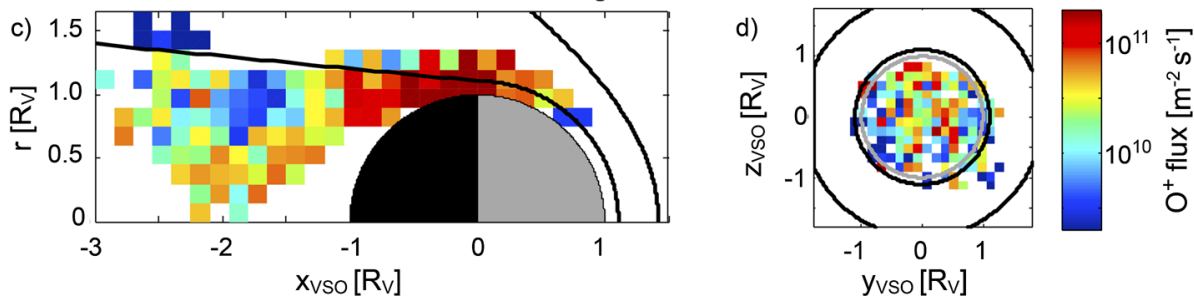

Fig. 21 The heavy ion flux measured by ASPERA-4/IMA is compared between stormy times (a, b), when ICMEs or CIRS are impacting the planet, and quiet times $(\mathbf{c}, \mathbf{d})$. The total escape rate increased by a factor of 1.9 during stormy times (after Edberg et al. 2011)

wind monitors provide many examples over the last decades of the possible structures that could be used with such models, which may be key to extrapolations of atmosphere loss back in time.

\section{3 "No Solar Wind" Event}

On 4 August, 2010, the observed Venus environment experienced an unusual condition, namely, a remarkably tenuous solar wind (Wei et al. 2012b). The density was estimated to be $\sim 0.2 \mathrm{~cm}^{-3}$, and the dynamic pressure $\sim 0.1 \mathrm{nPa}$. This situation lasted for 18 hours. Under the related low dynamic pressure condition, it is expected that the ionosphere is unmagnetized (e.g. Russell and Vaisberg 1983). This was the case of this event. As was observed, the dayside ionopause altitude also increased in response to the low incident pressure. The tail field became weak implying that the interplanetary field did not penetrate into the wake. The heavy ions, usually accelerated to the keV range in the plasmasheet, were not identified; instead, lower energy (10-50 eV in the spacecraft frame) ions, that were gravitationally bound to Venus, were detected along the VEX trajectory. Wei et al. (2012b) concluded that these are a part of the ionospheric component extended toward the nightside. These signatures are consistent with pressure balance between the Venusian ionosphere and the extremely low solar wind dynamic pressure. The induced magnetosphere is extended because of the relatively high ionospheric pressure, together with more effective shielding of the ionosphere against the interplanetary field. Then, inside the ionosphere, less effective mass loading and ionospheric ion acceleration causes the observed absence of the high energy ion fluxes. Thus this particular 'extreme' has the result of reducing planetary ion escape through the plasma sheet. 

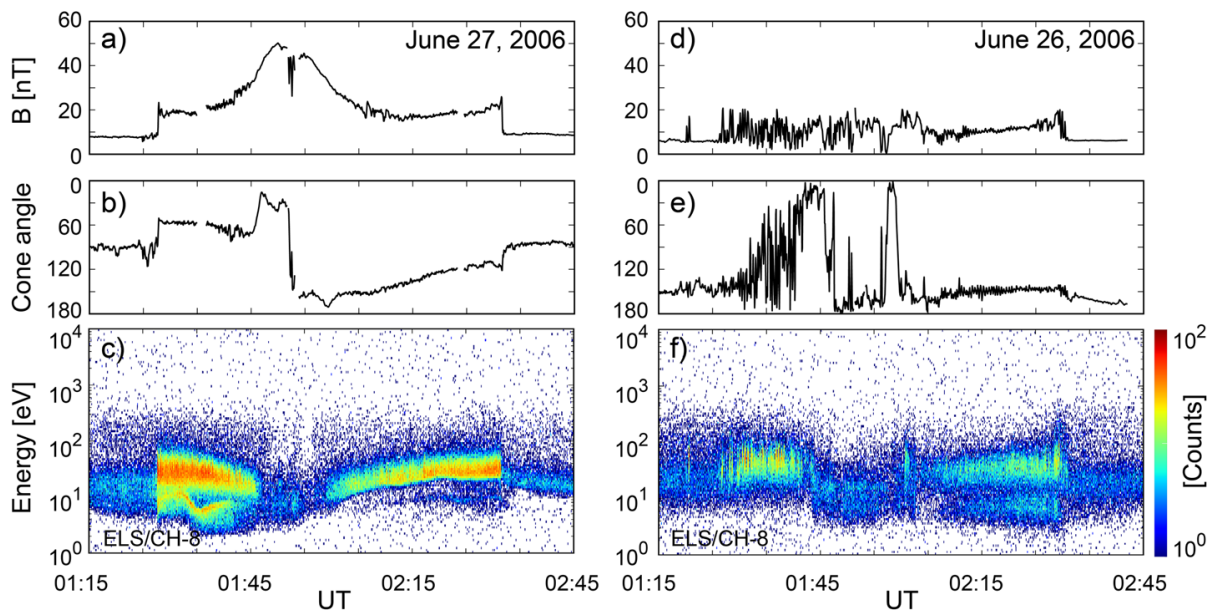

Fig. 22 Magnetic field strength $(\mathbf{a}, \mathbf{d})$, cone angle $\left(=\cos ^{-1}\left(B_{x} / B\right) ; \mathbf{b}, \mathbf{e}\right)$ and electron energy spectra $(\mathbf{c}, \mathbf{f})$ measurements recorded on consecutive days discussed in Zhang et al. (2009). (a-c) The induced magnetosphere is clearly formed when the upstream magnetic field is perpendicular (cone angle being $\sim 90^{\circ}$ outside of the interaction region). (d-f) The induced magnetosphere lacks the bulk of the magnetic field. In this period, the upstream magnetic field is dominated by the $B_{x}$ component (cone angle being $\sim 150^{\circ}$ )

\subsection{Interaction Without the Solar Wind Convective Electric Field}

On rare occasions, the interplanetary magnetic field is nearly aligned with the solar wind flow. Under these circumstances the convection electric field is strongly reduced and the poloidal component of the induced field should be dominant (see earlier discussion of induced field configurations). In this case the entire induced magnetosphere configuration is affected. The pileup of magnetic field on the dayside is barely observable and the clear magnetic structure of the magnetotail disappears (Zhang et al. 2009), although even in this situation a solar wind wake cavity/void is formed (Dubinin et al. 2013a).

Du et al. (2009) and Zhang et al. (2009) reported a remarkable example of this condition that occurred on 26 June, 2006. On that day, the entire Venus induced magnetosphere effectively disappeared. The magnetic field inside the magnetosheath was heavily fluctuating, and without the bulk increase of the field in the subsolar magnetic pileup/barrier region (Du et al. 2009). In the orbit after that, the normal induced magnetosphere was recovered, showing the typical structure. In both cases, the upstream IMF conditions were stable and the strengths were nominal, only the directions were different (Fig. 22). Du et al. (2010) concluded that the related observed difference in the fluctuations in the magnetosheath can be explained by the different subsolar bow shock types, namely quasi-parallel and quasi-perpendicular shocks for the flow-aligned and normal cases respectively (Song and Russell 1997). For the parallel case, almost the entire subsolar shock surface is quasi-parallel, creating the intense fluctuations (e.g. Luhmann et al. 1983).

Even though the parallel IMF conditions are rare, the distinct disappearing magnetosphere brings the strong influence of the solar wind at Venus. Under such circumstances the solar wind can easily access the Venus ionosphere/atmosphere, and the usual processes of ion acceleration and escape will change. Using MHD simulations, Zhang et al. (2009) calculated the atmospheric loss rate dependence on the IMF angle; the rate increased by $75 \%$ if the angle was changed from $11^{\circ}$ to $0^{\circ}$. Using a hybrid model, Liu et al. (2009) similarly 
concluded that the loss rate became higher, by $\sim 30 \%$, for a Parker spiral IMF $\left(36^{\circ}\right)$ compared to a purely perpendicular IMF. Masunaga et al. (2013) applied the statistical approach to the observations, using MAG and ASPERA-4 data to determine the loss rate dependence on the IMF conditions. They collected 167 cases of perpendicular conditions $\left(>60^{\circ}\right)$ and 82 cases of parallel conditions $\left(<30^{\circ}\right)$, calculating loss rates by integrating the heavy ion flux. They inferred escape rates of $(5.8 \pm 2.9) \times 10^{24} \mathrm{~s}^{-1}$ for the perpendicular IMF case and $(4.9 \pm 2.2) \times 10^{24} \mathrm{~s}^{-1}$ for the parallel case. As the difference is within an uncertainty range, they concluded that the dependence of the heavy ion loss rate on the upstream IMF direction is insignificant. A clear difference was, however, found in the spatial distribution of the heavy ion flux relative to the magnetic field. A single interval of heavy ion flux was simultaneously seen with a plasmasheet crossing along orbits in the perpendicular cases, while multiple fluxes and plasmasheet crossings were observed in the parallel cases. Masunaga et al. (2011) suggested that a stable, single plasmasheet along the interplanetary electric field direction is formed under the perpendicular IMF condition, while multiple, scattered plasmasheets appear to be formed for the parallel condition. The planetary ion acceleration mechanisms are therefore different even though the ion loss rate is about the same.

In general, these unusual conditions help to highlight the role(s) numerical simulations can play in exploring the wide parameter space of the Venus-solar wind interaction, present and past.

\section{Modeling Work}

\subsection{Numerical Simulations of the Global Solar Wind Interaction}

By the time the Venus Express mission arrived at Venus, the ability to numerically simulate the global solar wind interaction and its consequences was well-advanced compared to the PVO era. Several reviews of the state of the art on such models are available (e.g. Kallio et al. 2011; Ledvina et al. 2008), so that we focus here on specific applications to understanding the results from VEX. The two main types of models applied to the Venus interaction are MHD models, which treat the solar wind and ionspheric plasmas as magnetized fluids, and hybrid models, which treat the plasma electrons as a fluid but the ions as particles. There are advantages to both of these approaches. The fluid models capture the large-scale attributes of the solar wind disturbance including the shock, magnetosheath/magnetic barrier and ionosphere obstacle - and include production of planetary ions outside of the obstacle boundary (the ionopause) as well as inside, while the hybrid models capture some of the consequences of the large ion gyroradii of the heavy planetary species. The fluid models generally have higher spatial resolution ( $\sim 5 \mathrm{~km}$ in radius), especially close to the inner boundary of the simulation (typically at or above $\sim 100 \mathrm{~km}$ ), allowing them to simulate some of the ionospheric consequences of the solar wind interaction. On the other hand, the hybrid models bring out asymmetries in the interaction caused by the fact that the unmagnetized obstacle of Venus has a comparable scale to the major $\left(\mathrm{O}^{+}, \mathrm{O}_{2}{ }^{+}, \mathrm{CO}_{2}{ }^{+}\right)$planetary ion species involved in that interaction. Both of these models complement the work on atmosphere/ionosphere models described in the accompanying article by Gérard et al. (2017). However, up to now there has been no specific attempt to merge the models in these different, though overlapping, domains. In general the global solar wind interaction models described here use simplified neutral atmospheres (including exospheres), with no internal dynamics, and simplified ion production descriptions. 


\subsubsection{MHD Models}

The main state-of-the-art MHD models that have been applied to the understanding of the Venus solar wind interaction in the VEX era are those by Terada et al. (2009) and Ma et al. (2013). Each one has been compared to some limited observations, suggesting their usefulness for interpreting one is seen in a global context and with physical insights, but has yet to be fully exploited.

The Terada et al. (2009) model is a single fluid, multispecies type (e.g. see Kallio et al. 2011) involving 10 ion species including both solar wind and planetary hydrogen ions. Single fluid treatments constrain all particle species to move at a single velocity, even though their descriptions are dictated by separate continuity equations. This model built on techniques used in an earlier, simpler global simulation by Tanaka (1993) and Tanaka and Murawski (1997), and was designed to achieve low numerical diffusion. A spherically symmetric description is used for the thermospheric (thermal) part of the atmosphere, as well as for the hydrogen exosphere or corona, while the oxygen exosphere (see Gerard et al. for a discussion of its origins) falls off with solar zenith angle according to a standard cosine to the one-half dependence. All key ion production terms are included in the multispecies MHD equations that are solved: photoionization, electron impact ionization, and charge exchange. At the inner model boundary at $120 \mathrm{~km}$ the magnetic field and velocity are set to zero, while solar wind hydrogen ion inflow is assumed at an upstream boundary at 8.1 Venus radii-and outflow elsewhere. The spatial resolution associated with the calculation grid is $\sim 6-10 \mathrm{~km}$ near the inner boundary and grows to $\sim 1000 \mathrm{~km}$ in the outer grid. The model was run for specific solar (EUV) ionizing flux and solar wind conditions, including a Parker Spiral magnetic field of strength $\sim 6 \mathrm{nT}$. Together with the slow solar wind speed used, $350 \mathrm{~km}$, these assumptions are consistent with a quiet solar wind interaction under moderate solar activity levels.

The Terada et al. (2009) simulation produces an upstream bow shock, magnetosheath and magnetic barrier, and induced magnetotail with plasma domain morphologies and magnetic fields consistent with what was observed on PVO. In particular, the positions of the bow shock and magnetic barrier interface with the ionosphere, the ionopause, are like those observed during PVO's solar maximum if the assumed ionosphere and solar wind conditions are considered. The subsolar pressure balance inferred from the spacecraft measurements, indicating conversion from mainly solar wind dynamic pressure to ionospheric thermal plasma pressure, is found along the subsolar axis of the model. The observed ionospheric ion escape into the wake and magnetotail is also obtained. A great advancement made by this model is the ability to see how the ionosphere interface and bulk ionosphere characteristics are altered by the solar wind interaction. In a purely photochemical model of the ionosphere, isolated from the solar wind, a specific scale height would be obtained that is dictated by production, transport, and the outer boundary condition imposed (e.g. Cravens 1992; Fox and Sung 2001). However, with the solar wind interaction, the momentum equations have other forces besides gravity and thermal pressure gradients. Terada et al. (2009) showed how their subsolar model altitude profiles of ionospheric ion density and magnetic field behave. They obtained a classic solar maximum 'unmagnetized ionosphere' condition where the subsolar pressure balance between the magnetic barrier pressure and the ionospheric thermal pressure occurs at $\sim 450 \mathrm{~km}$, well above the altitude (at $\sim 250 \mathrm{~km}$ ) where collisions of electrons with neutrals, together with the downward ionospheric plasma velocities, enable inward diffusion of magnetic barrier fields. They also used the results to demonstrate that the trans-terminator ionospheric flows observed on PVO as a result of day-to-night pressure gradient forces are also simulated in a self-consistent way. 
The more recent Ma et al. (2013) MHD model is also a single fluid, multispecies simulation. It is based on the BATS-R-US code approach (Powell et al. 1999), with a spherical grid and graduated spatial resolution that diminishes to $\sim 5 \mathrm{~km}$ near its inner boundary at $100 \mathrm{~km}$ altitude. This model includes ionospheric $\mathrm{O}^{+}, \mathrm{O}_{2}{ }^{+}$and $\mathrm{CO}_{2}{ }^{+}$as well as solar wind hydrogen ions and a parker spiral magnetic field, and was run for solar maximum and minimum conditions (solar EUV fluxes and solar wind parameters), as well as a specific case for VEX magnetometer data comparison. Like the Terada et al. (2009) simulation, the Ma et al. (2013) model uses spherically symmetric neutral atmosphere descriptions and assumes all ionization production processes (photoionization, electron impact and charge exchange), but neglects the exospheric components of the neutral atmosphere. Another difference is that the innermost boundary of the simulation is treated as an absorber, assuming zero gradients there for both flow and field vectors. These simulations also reproduce the general bow shock and magnetosheath/magnetic barrier and magnetotail features, additionally finding solar activity modifications of the shock location consistent with changes seen in the PVO and VEX observations. Moreover, the models' subsolar conditions, also seen in some earlier results of Tanaka (1998), exhibit the lowered ionopause and magnetized subsolar ionosphere condition for the solar minimum case when the incident solar wind pressure pushes the nominal pressure balance altitude into the collisional, downward ionospheric velocity domain. In general, the authors conclude that the combination of a weaker ionosphere and the solar minimum solar wind conditions contribute to the obtained different solar activity model differences. A specific VEX pass was also selected by these authors for more detailed comparison with model results, with upstream and solar conditions set according to VEX measurements for that time. Magnetic field vector rotations and magnitudes throughout the near-periapsis section of the VEX orbit were well-reproduced, including the shock and magnetic barrier behaviors (Fig. 23). Corresponding model plasma attributes were obtained but not compared with data. However, the Ma et al. (2013) model was also applied toward understanding the special condition of a solar wind flow-aligned interplanetary field that causes the occasion disappearance of the magnetic barrier and standard draped-field magnetotail (Zhang et al. 2009).

While these MHD models have not been fully exploited for understanding the global context and physical settings of the observations at Venus, there is increased interest in instead applying multifluid, multispecies models in which each ion species in the simulation has its own velocity. This is motivated mainly by the observations on VEX which show evidence for solar wind convection electric field-related asymmetries in the solar wind interaction (e.g. Dubinin et al. 2013b, 2014; Zhang et al. 2010, 2015). Most recently, Villarreal et al. (2015) explain an observed interplanetary field orientation bias in the north polar region magnetic field strength found by VEX using a multifluid version of the Ma et al. (2013) model. Of course global hybrid models, described below, have long ago illustrated such asymmetries, but generally without many of the details the higher spatial resolution MHD models can provide-including the ionosphere descriptions.

\subsubsection{Global Hybrid Models}

The development of a global hybrid model for the Venus-solar wind interaction by Kallio et al. (2006) around the time of VEX's arrival at Venus spear-headed a range of studies motivated by observations. This model, which focuses on hydrogen ions of both solar wind and planetary origin, and oxygen ions of planetary origin, was designed to investigate the regions and processes above the bulk ionosphere where ion kinetic effects have most influence. By not focusing on low altitudes, a number of simplifying assumptions can be made, including 

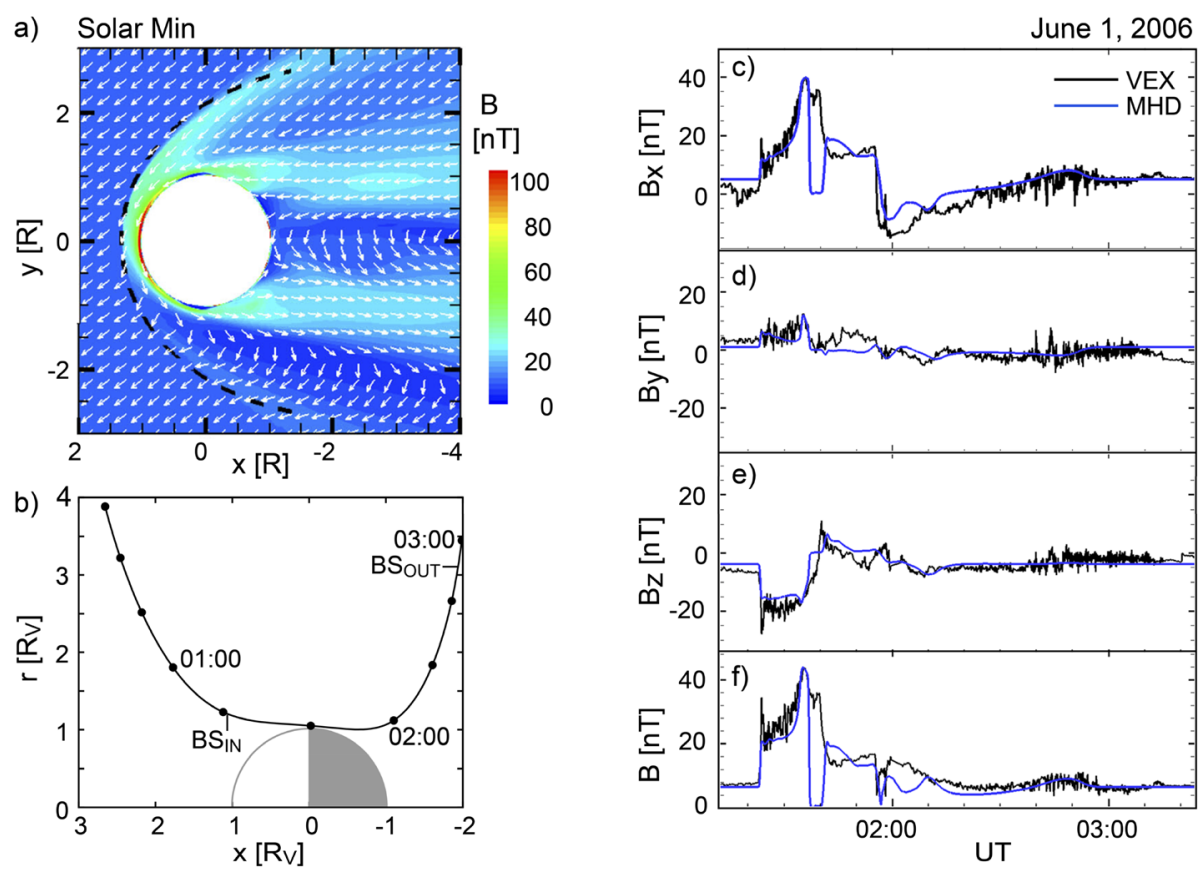

Fig. 23 Results from the Ma et al. (2013) MHD model. (a) The simulated global magnetic field pattern is shown. The field strength is color coded and the direction indicated by the white arrows. Along the trajectory of VEX (b), a comparison between the model magnetic field and the magnetometer data recorded along the trajectory of Venus Express is plotted (c-f). Figure is adopted from Ma et al. (2013)

reduced spatial resolution $(\sim 600 \mathrm{~km})$, which keeps global hybrid models within reasonable computational requirements. The Kallio et al. (2006) model injects a cold, thermal ionospheric ion population into the simulation grid at an inner boundary at $\sim 400 \mathrm{~km}$ (near the nominal subsolar Venus ionopause). Additional, in-situ production of planetary ions above that boundary is included from the neutral atmosphere (including the exosphere) that extends above that altitude. The thermal ions are introduced at solar zenith angle-dependent rates consistent with their production by photoionization, with photoionization production is also assumed in the optically thin atmosphere outside the inner boundary. Given the production of ions and their subsequent single particle motion in the magnetic and electric fields introduced by the flowing solar wind, the calculated ion trajectories show a complex range of behaviors. Some ions impact the inner simulation boundary, where they are removed from the simulation. The hybrid simulation also produces the main structures of the solar wind interaction obtained in the MHD models, including bow shock, magnetosheath/magnetic pile-up region, and induced magnetotail. The major difference is that the results are not symmetric about the magnetic equator due to the combined effects of ion absorption at the inner boundary, the large heavy ion gyroradius escaping planetary ions, and the separate treatment of the electron 'fluid'.

The hybrid model results have been compared to individual VEX passes (Kallio et al. 2008), to PVO magnetic field observations (Jarvinen et al. 2008), to the study of flowaligned interplanetary field cases (Liu et al. 2009), to the analysis of the different patterns of planetary $\mathrm{H}^{+}$and $\mathrm{O}^{+}$escape (Jarvinen et al. 2010), and to study the effect of the interplanetary field flow-aligned component $\left(B_{x}\right)$ on escape rates (Jarvinen et al. 2013). An 
Fig. 24 Test particle trajectories of (a) $\mathrm{H}^{+}$, (b) $\mathrm{He}^{+}$, (c) $\mathrm{O}^{+}$, and (d) $\mathrm{O}_{2}{ }^{+}$pickup ions in a hybrid fields of Venus-solar wind interaction, viewed from the flank side. The background coloring shows the $\mathrm{O}^{+}$density (adopted from Jarvinen et al. 2016)
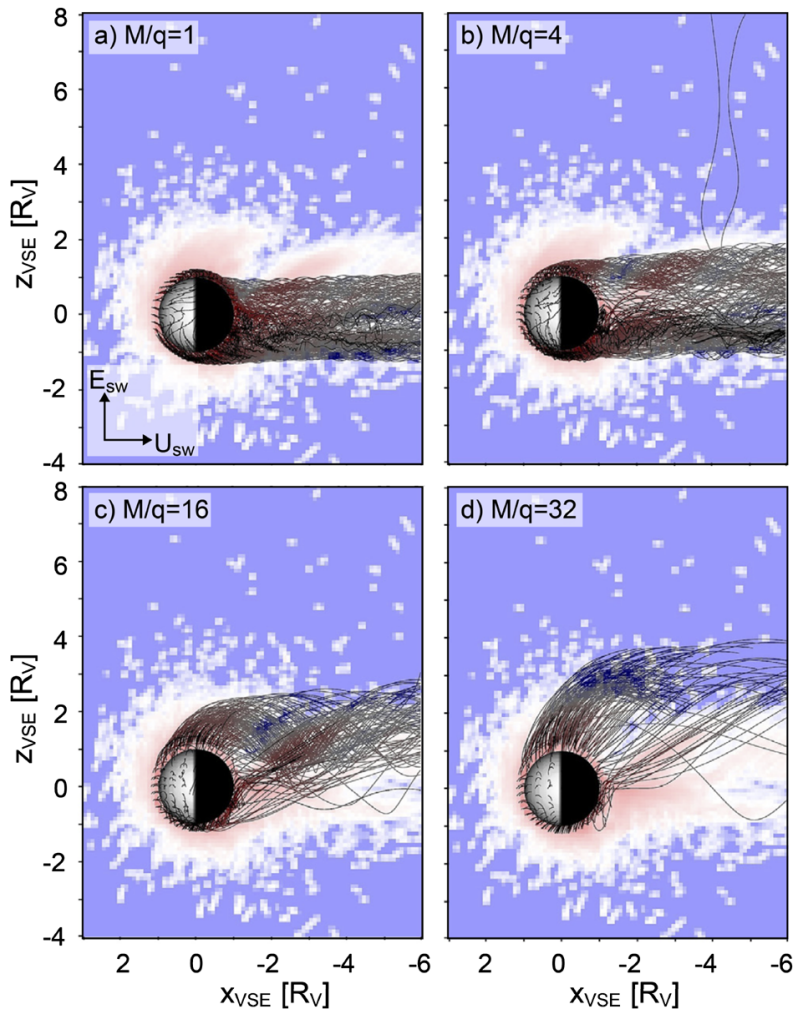

example of modeled plasma escape trajectories is shown in Fig. 24. Hybrid models have also been used to interpret the many observed solar wind electric field related asymmetries such as the magnetosheath field draping pattern differences in the north and south magnetic hemispheres (Zhang et al. 2010; Du et al. 2013).

\subsection{Other Related Models}

\subsubsection{Test Particle Models}

The results of both the MHD and hybrid models have also been applied to modeling other details of the Venus solar wind interaction, giving further information about its consequences. For example, test particle ion tracings in MHD simulation fields have been used to investigate energy spectra and spatial features of $\mathrm{O}^{+}$pickup ions observed on VEX during the passage of Interplanetary Coronal Mass Ejections (Luhmann et al. 2006). Most recently, Curry et al. (2015) used the Ma et al. (2013) MHD model results to analyze the patterns of $\mathrm{O}^{+}$ion escape and energy deposition into the ionosphere, comparing the Venus case to Mars. In other studies using the hybrid model, Gunell et al. (2007) calculated the global pattern of Venus X-ray emission that one would expect from the charge exchange reactions of highly ionized heavy solar wind ions in the Venus upper atmosphere. The Chandra observatory observed such X-rays (Dennerl 2008). Galli et al. (2008) describe the use of the models to understand the ENA emissions observed on VEX in the Venus wake. 


\subsubsection{Monte Carlo Models}

Localized/regional modeling has also been applied to understand the physics at the interface between the incident solar wind and upper atmosphere. Shematovich et al. (2014) considered the effects of an induced ionospheric magnetic field on the ability of solar wind ions to penetrate into the Venus atmosphere with a Monte-Carlo simulation of the interaction between the two. They found that such penetration helps to reflect the solar wind ions, results suggesting that absorption would be minimized with the ionosphere is magnetized due to the condition of either low solar EUV fluxes or high solar wind dynamic pressures. These results help to explain the inference of low solar wind absorption observed on VEX.

\subsection{Future Possibilities for Modeling contributions}

Many other facets of the Venus-solar wind interaction and its effects are still waiting to be explored that can be enabled by these and other models. In particular, the merging of the state of the art upper atmosphere and ionospheric chemistry models (see Gérard et al. 2017 in this volume) with the solar wind interaction models can be used to investigate such questions as the role of neutral winds on the solar wind interaction and the consequences of the heavy pickup ion precipitation (e.g. sputtering-see Luhmann and Kozyra 1991). As mentioned above, the more explicit treatment of Venus itself in the global models could reveal some details related to possible leakage of interplanetary fields into the interior. Indeed the ionospheric holes (e.g. Collinson et al. 2014b) are not yet well-reproduced by the models. The matter of bulk removal of ionospheric ions by fluid instabilities, and their importance for ion escape (e.g. Moestl et al. 2011) is also still under examination. Finally, understanding the extent to which the long-term evolution of the Venus atmosphere is related to the solar wind interaction (e.g. Lammer et al. 2008) will ultimately be based on such modeling to constrain the possible impacts.

\section{Summary of the Venus Express Findings}

As seen in the previous sections, Venus Express results produced many new insights beyond PVO about both the details of the plasma environment and the north polar region in particular. It observed the bow shock and ionospheric boundaries of Venus and associated phenomena (like plasma waves and hot flow anomaly) at a level of detail not possible with PVO plasma instrumentation. It repeatedly sampled the high latitude plasma interaction down to altitudes of $\sim 250 \mathrm{~km}$ through a long and deep solar minimum, and probed the magnetic field to $\sim 130 \mathrm{~km}$ as it made its final entry. VEX found that planetary ion escape in the solar wind wake and adjacent magnetosheath is well-organized by the magnetosheath magnetic field draping and the solar wind convection electric field direction. The atmospheric escape with energy of $\sim 10 \mathrm{eV}$ to $\sim 10 \mathrm{~s}$ of $\mathrm{keV}$ around the VEX orbit were found to be roughly in line with some earlier estimates based on PVO's less extensive observations, though under the different solar activity. However, because of the capability of the ion mass analysis, the VEX ion spectrometer found that the ratio of $\mathrm{H}^{+}$to $\mathrm{O}^{+}$escape is stochastically to the water molecule, implying the water loss from the Venus atmosphere as of now. As for the reverse process of absorption, VEX was also able to detect solar wind hydrogen and helium deposition in the upper atmosphere-allowing evaluation of accretion rates for comparisons-; though the shielding by the induced magnetosphere was quite strong that the precipitation of the solar wind particles were prohibited to enter into the atmosphere. VEX provided a unique 
perspective on the large-scale magnetization of the solar minimum polar ionosphere, which, like that of the low latitude, high solar activity ionosphere seen on PVO, is sensitive to the incident solar wind pressure. The first-ever energetic neutral atom measurements revealed the neutral exosphere and solar wind plasma interaction near Venus. Other investigations with the magnetometer data found solar wind convection electric field-related asymmetries in both the sheath and polar ionospheric field strength and in sheath field draping as well. The latter suggested magnetic reconnection occurs in the Venus wake between adjacent induced tail lobes, likely influencing planetary ion escape there. The lightning was confirmed by the magnetometer data as well. Finally, the last weeks of exceptionally low altitude measurements confirmed the absence of average magnetic fields of more than a few nT strength in the deep polar ionosphere.

\section{Future Missions and Open Questions}

Despite Venus is being explored for more than 55 years, being much longer than Mars, no prospects currently exist for a follow-on similarly focused on the solar wind interaction and related atmosphere escape with the end of the Venus Express mission. A dedicated aeronomy and solar wind interaction mission similar to MAVEN (Mars Atmosphere and Volatile Evolution Mission) launched to Mars in 2013 (Jakosky et al. 2015) is still to be conducted. Such a VEX-like mission with lower periapsis that drifts in latitude around Venus so as to cover both the north and south poles, noon and midnight sectors, and terminator regions - and of course that observes during a full solar cycle is desired. It has the prospect of eliminating the ambiguities we are left with concerning solar cycle influences and 3D magnetic field geometry under low and high solar EUV conditions after PVO and VEX. It would also be important to sample a much more active solar cycle than what VEX has experienced, with its modern instrumentation. The influence of space weather 'storms' on ion escape processes and rates may be essential for understanding evolutionary impacts. It is notable that exoplanet researchers sometimes invoke Venus as a model for remote sensing planets that seem to have comet-like interactions with their host stars. As such, we would do well to continue the quest to understand the history of Venus, and the role the solar wind interaction has played in that history.

On the other hand, Venus is an important body for swing-by maneuvers for the route to the inner (Mercury), and outer solar system planets (e.g. Jupiter, Saturn). Therefore, a number of fly-bys with spacecraft very-well equipped with plasma and field instrumentation is expected. The coming fly-by missions and missions relevant for the solar wind investigation are, for example, BepiColombo, Solar orbiter, Solar probe plus, and JUICE. Such flyby missions provide only a short-time range data, but on the other hand, the benefit is that we can spend more time for preparation of measurement for the flyby event with an intensive collaboration between sensors.

Like for other non-magnetized planets, the field of the solar wind interaction with Venus is directly related to fundamental scientific topics such as planetary atmosphere evolution and escape to space, physics of induced magnetospheres, upper atmosphere physics and ionosphere. Due to the absence of the local magnetizations as on Mars and the Moon, precise magnetic field measurements and understanding of the ionospheric currents can be used to define the magnetic field from the induced current in the crust and thus reveal the conductivity of the mantle, which in turn related to the water content in the crust. The latter is the key parameter to understand the evolution of water on the currently dry Venus. Table 2 sums 
Table 2 Open science questions for the future missions to Venus

\begin{tabular}{ll}
\hline Open scientific questions $\quad$ Required investigations & $\begin{array}{l}\text { Measurements and mission } \\
\text { requirements }\end{array}$
\end{tabular}

Science topic: Atmospheric evolution and escape

How did Venus interact with the solar wind during the early Sun? What were the planetary ion's escape rates?
Planetary ion distribution in the Venus induced magnetosphere during extreme solar and solar wind conditions

Global MHD and/or hybrid models coupled with the variable ionosphere

What is the fate of the primordial nitrogen in the Venus atmosphere? How much escaped to space?

Is sputtering an effective mechanism to remove the atmosphere?

\section{Science topic: Physics of induced magnetospheres}

How does the induced magnetosphere of Venus response to the variable solar wind, for example, sectorial boundary crossing

How does reconnection operate in the induced magnetosphere tails?

What is the role of waves in the planetary ion heating and acceleration?

The mass composition of the escaping planetary ions?

Oxygen exosphere and its profiles. Non-thermal components

Planetary ion distribution in the Venus induced magnetosphere and magnetic field configurations

Study of the plasmoid formations

Characterized the waves power spectrum and ion distribution functions
Energetic particle measurements, UV monitors, upstream condition monitoring

Long-term sampling of the magnetosphere with particle and field instruments to achieve good statistics

Reliable mass separation $\mathrm{C}^{+}, \mathrm{N}^{+}$, $\mathrm{O}^{+}, \mathrm{O}_{2}^{+}, \mathrm{CO}_{2}^{+}, \mathrm{NO}^{+}, \mathrm{CO}^{+}$, $\mathrm{N}_{2}+, \mathrm{C}, \mathrm{N}$

UV spectroscopy. Next generation of ultra-low energy analyzers of neutrals

Long-term sampling of the magnetosphere with particle and field instruments to achieve good statistics

Sampling of the region from 0.5 to 3-5 $R_{\mathrm{V}}$ down-tail

Waves and $E$ and $B$-fields measurements combined with high time resolution particle measurements

Magnetic field measurements with high frequency sampling

Simultaneous particle measurements (neutral and ions) with field. Remote sensing by ENAs and UV spectroscopy in the same FoV

Magnetic field measurements from balloons and other flying platforms
Inductive magnetic fields at the bottom of the ionosphere question of the water content in the crust?

\section{Interior}

What is the conductivity of the
Global distribution of the whistler

Simultaneous in situ and remote measurements of neutral atoms, charged particles, and fields

up a list of open questions to be answered by the coming dedicated or relevant missions for each scientific topic.

The situation with missions to Venus resembles the Moon exploration when the active 60s and 70s followed by a long, almost 40 years, pause with just very few small missions launched. However, we are now witnessing a renaissance in the lunar studies. New capable missions bring entirely unexpected findings. Venus Express may be in this position of opening this new era. Its findings brought the Venus research to the new level and resulted in 
formulation of the new outstanding questions for next missions. The field of the solar windVenus interaction indeed involves science of the fundamental importance to be address on the all coming missions.

Open Access This article is distributed under the terms of the Creative Commons Attribution 4.0 International License (http://creativecommons.org/licenses/by/4.0/), which permits unrestricted use, distribution, and reproduction in any medium, provided you give appropriate credit to the original author(s) and the source, provide a link to the Creative Commons license, and indicate if changes were made.

\section{References}

M.H. Acuña, J.E.P. Connerney, P. Wasilewski et al., Magnetic field of Mars: summary of results from the aerobraking and mapping orbits. J. Geophys. Res., Planets 106(E10), 23,403-23,417 (2001). doi:10.1029/2000JE001404

C.J. Alexander, J.G. Luhmann, C.T. Russell, Interplanetary field control of the location of the Venus bow shock: evidence for comet-like ion pickup. Geophys. Res. Lett. 13, 917-920 (1986). doi:10.1029/ GL013i009p00917

H. Alfvén, On the theory of comet tails. Tellus IX, 92 (1957). doi:10.1111/j.2153-3490.1957.tb01855.X

U.V. Amerstorfer, N.V. Erkaev, D. Langmayr et al., On Kelvin-Helmholtz instability due to the solar wind interaction with unmagnetized planets. Planet. Space Sci. 55, 1811-1816 (2007). doi:10.1016/j.pss.2007. 01.015

B.J. Anderson, M.H. Acuna, D.A. Lohr et al., The magnetometer instrument on messenger. Space Sci. Rev. 131(1), 417-450 (2007). doi:10.1007/s11214-007-9246-007-7

G.B. Andrews, T.H. Zurbuchen, B.H. Mauk et al., The energetic particle and plasma spectrometer instrument on the messenger spacecraft. Space Sci. Rev. 131(1), 523-556 (2007). doi:10.1007/s11214-007-9272-5

A. Angsmann, M. Fränz, E. Dubinin et al., Magnetic states of the ionosphere of Venus observed by Venus express. Planet. Space Sci. 59, 327-337 (2011). doi:10.1016/j.pss.2010.12.004

M.A. Balikhin, T.L. Zhang, M. Gedalin et al., Venus express observes a new type of shock with pure kinematic relaxation. Geophys. Res. Lett. 35, L01103 (2008). doi:10.1029/2007GL032495

S. Barabash, A. Fedorov, J.J. Sauvaud et al., The loss of ions from Venus through the plasma wake. Nature 450, 650-653 (2007a). doi:10.1038/nature06434

S. Barabash, J.-A. Sauvaud, H. Gunell et al., The Analyser of Space Plasmas and Energetic Atoms (ASPERA4) for the Venus express mission. Planet. Space Sci. 55, 1772-1792 (2007b). doi:10.1016/j.pss.2007. 01.014

C. Bertucci et al., MGS MAG/ER observations at the magnetic pileup boundary of Mars: draping and low frequency waves. Adv. Space Res. 33, 1938-1944 (2004). doi:10.1016/j.asr.2003.04.054

C. Bertucci, N. Achilleos, M.K. Dougherty, R. Modolo, A.J. Coates, K. Szego, A. Masters, Y. Ma, F.M. Neubauer, P. Garnier, J. Wahlund, D.T. Young, The magnetic memory of Titan's ionized atmosphere. Science 321(5895), 1475-1478 (2008). doi:10.1126/science.1159780

C. Bertucci, F. Duru, N. Edberg et al., The induced magnetospheres of Mars, Venus, and Titan. Space Sci. Rev. 162, 113-171 (2011). doi:10.1007/s11214-011-9845-1

H.K. Biernat, N.V. Erkaev, U.V. Amerstorfer et al., Solar wind flow past Venus and its implications for the occurrence of the Kelvin-Helmholtz instability. Planet. Space Sci. 55, 1793-1803 (2007). doi:10.1016/j.pss.2007.01.006

S.W. Bougher, D.M. Hunten, R.J. Phillips (eds.), Venus II: Geology, Geophysics, Atmosphere, and Solar Wind Environment (Univ. of Arizona Press, Tucson, 1997)

L.H. Brace, R.F. Theis, W.R. Hoegy et al., The dynamic behavior of the Venus ionosphere in response to to solar wind interactions. J. Geophys. Res. 85, 7663-7678 (1980). doi:10.1029/JA085iA13p07663

L.H. Brace, R.T. Theis, W.R. Hoegy, Plasma cloud above the ionopauser of Venus and their implications. Planet. Space Sci. 30(1), 29-37 (1982a). doi:10.1016/0032-0633(82)90069-1

L. Brace, R. Theis, H. Mayr et al., Holes in the nightside ionosphere of Venus. J. Geophys. Res. 87, 199-211 (1982b). doi:10.1029/JA087iA01p00199

L.H. Brace, W.T. Kasprzak, H.A. Taylor et al., The ionotail of Venus-its configuration and evidence for ion escape. J. Geophys. Res. 92(1), 15-26 (1987). doi:10.1029/JA092iA01p00015

S.H. Brecht, Magnetic asymmetries of unmagnetized planets. Geophys. Res. Lett. 17, 1243-1246 (1990). doi:10.1029/GL017i009p01243

S.H. Brecht, J.R. Ferrante, Global hybrid simulation of unmagnetized planets: comparison of Venus and Mars. J. Geophys. Res. 96, 11209-11220 (1991). doi:10.1029/91JA00671 
T.K. Breus, Venus: review of present understanding of solar wind interaction. Space Sci. Rev. 23, 253-279 (1979). doi:10.1007/BF00173812

H.S. Bridge, A.J. Lazarus, G.L. Siscoe et al., Interaction of the solar wind with Venus, in Solar-Wind Interaction with the Planets, Mercury, Venus, and Mars, ed. by N.F. Ness (NASA, Washington, 1976), pp. 63-80

L. Chai, M. Fränz, W. Wan et al., IMF control of the location of Venusian bow shock: the effect of the magnitude of IMF component tangential to the bow shock surface. J. Geophys. Res. 119, 9464-9475 (2014). doi:10.1002/2014JA019878

L. Chai, W. Wan, M. Fränz et al., Solar zenith angle-dependent asymmetries in Venusian bow shock location revealed by VEX. J. Geophys. Res. Space Phys. 120, 4446-4451 (2015). doi:10.1002/2015JA021221

A.J. Coates, R.A. Frahm, D.R. Linder et al., Ionospheric photoelectrons at Venus: initial observations by ASPERA-4 ELS. Planet. Space Sci. 56, 802-806 (2008). doi:10.1016/j.pss.2007.12.008

A.J. Coates, S.M.E. Tsang, A. Wellbrock et al., Ionospheric photoelectrons: comparing Venus, Earth, Mars and Titan. Planet. Space Sci. 59, 1019-1027 (2011). doi:10.1016/j.pss.2010.07.016

A.J. Coates, A. Wellbrock, R.A. Frahm et al., Distant ionospheric photoelectron energy peak observations at Venus. Planet. Space Sci. 113-114, 378-384 (2015). doi:10.1016/j.pss.2010.07.016

G.A. Collinson, D.G. Sibeck, A. Masters et al., Hot flow anomalies at Venus. J. Geophys. Res. 117, A04204 (2012a). doi:10.1029/2011JA017277

G.A. Collinson, L.B. Wilson, D.G. Sibeck et al., Short large-amplitude magnetic structures (SLAMS) at Venus. J. Geophys. Res. 117, A10221 (2012b). doi:10.1029/2012JA017838

G.A. Collinson, D.G. Sibeck, A. Masters et al., A survey of hot flow anomalies at Venus. J. Geophys. Res. 119, 978-991 (2014a). doi:10.1002/2013JA018863

G.A. Collinson, A. Fedorov, Y. Futaana et al., The extension of ionospheric holes into the tail of Venus. J. Geophys. Res. 119, 6940-6953 (2014b). doi:10.1002/2014JA019851

B.V. Connor, Space magnetics: Mariner V magnetometer experiment. IEEE Trans. Magn. 4(3), 391-397 (1968). doi:10.1109/TMAG.1968.1066331

T.E. Cravens, Ionospheric models for Venus and Mars, in Venus and Mars: Atmospheres, Ionospheres, and Solar Wind Interactions, ed. by J.G. Luhmann, M. Tatrallyay, R.O. Pepin. AGU Monograph, vol. 66 (American Geophysical Union, Washington, 1992), pp. 277-288. doi:10.1029/GM066p0277

S.M. Curry, J. Luhmann, Y. Ma et al., Comparative pick-up ion distributions at Mars and Venus: consequences for atmospheric deposition and escape. Planet. Space Sci. 115, 35-47 (2015). doi:10.1016/j.pss. 2015.03.026

J.T.M. Daniels, C.T. Russell, R.J. Strangeway et al., Whistler mode bursts in the Venus ionosphere due to lightning: statistical properties using VEX magnetometer observations. J. Geophys. Res. 117, E04004 (2012). doi:10.1029/2011JE003897

E.M. De Gouveia Dal Pino, G. Kowal, L.H.S. Kadowaki et al., Int. J. Mod. Phys. D 19, 729 (2010). doi:10. $1142 / \mathrm{S} 0218271810016920$

M. Delva, T.L. Zhang, M. Volwerk et al., First upstream proton cyclotron wave observations at Venus. Geophys. Res. Lett. 35, L03105 (2008a). doi:10.1029/2007GL032594

M. Delva, T.L. Zhang, M. Volwerk et al., Proton cyclotron waves in the solar wind at Venus. J. Geophys. Res. 113, E00B06 (2008b). doi:10.1029/2008JE003148

M. Delva, C. Mazelle, C. Bertucci et al., Proton cyclotron wave generation mechanisms upstream of Venus. J. Geophys. Res. 116, A02318 (2011). doi:10.1029/2010JA015826

M. Delva, C. Bertucci, M. Volwerk et al., Upstream proton cyclotron waves at Venus near solar maximum. J. Geophys. Res. 120, 344-354 (2015). doi:10.1002/2014JA020318

K. Dennerl, X-rays from Venus observed with Chandra. Planet. Space Sci. 56, 1414-1423 (2008). doi:10. 1016/j.pss.2008.03.008

A.P. Dimmock, S.N. Walker, T.L. Zhang et al., Spatial scales of the magnetic ramp at the Venusian bow shock. Ann. Geophys. 29, 2081-2088 (2011). doi:10.5194/angeo-29-2081-2011

Sh.Sh. Dolginov, E.G. Eroshenko, L.N. Zhuzgov, Study of magnetic fields by means of the Venera-4 space probe. Kosmich. Issled. 6, 561 (1968)

Sh.Sh. Dolginov, Y.G. Yeroshenko, L. Davis, On the nature of the magnetic field near Venus. Kosmich. Issled. 7, 747 (1969)

Sh.Sh. Dolginov, E. Dubinin, Ye.G. Yeroshenko et al., On the configuration of the field in the magnetic tail of Venus. Cosm. Res. 19, 624 (1981)

T.M. Donahue, J.H. Hoffman, R.R. Hodges et al., Venus was wet-a measurement of the ratio of deuterium to hydrogen. Science 216, 630-633 (1982). doi:10.1126/science.216.4546.630

G.D. Dorrian, A.R. Breen, J.A. Davies et al., Transient structures and stream interaction regions in the solar wind: results from EISCAT interplanetary scintillation, STEREO HI and Venus express ASPERA-4 measurements. Sol. Phys. 265, 207-231 (2010). doi:10.1007/s11207-010-9599-z 
M.K. Dougherty, D. Kellock, D.J. Southwood et al., The Cassini magnetic field investigation. Space Sci. Rev. 114, 331-383 (2004). doi:10.1007/s11214-004-1432-2

J. Du, T.L. Zhang, C. Wang et al., Magnetosheath fluctuation at Venus for two extreme orientations of the interplanetary magnetic field. Geophys. Res. Lett. 36, L09102 (2009). doi:10.1029/2009GL037725

J. Du, T.L. Zhang, W. Baumjohann et al., Statistical study of low-frequency magnetic field fluctuations near Venus under the different interplanetary magnetic field orientations. J. Geophys. Res. 115, A12251 (2010). doi:10.1029/2010JA015549

J. Du, C. Wang, T.L. Zhang et al., Asymmetries of the magnetic field line draping shape around Venus. J. Geophys. Res. Space Phys. 118, 6915-6920 (2013). doi:10.1002/2013JA019127

E. Dubinin, M. Fraenz, Magnetotails of Mars and Venus, in Magnetotails in the Solar System. AGU Geophysical Monograph, vol. 207 (2015). doi:10.1002/9781118842324.ch3

E. Dubinin, M. Fraenz, A. Fedorov et al., Ion energization and escape on Mars and Venus. Space Sci. Rev. 162, 173-211 (2011). doi:10.1007/s11214-011-9831-7

E. Dubinin, M. Fraenz, J. Woch et al., Bursty escape fluxes in plasma sheets of Mars and Venus. Geophys. Res. Lett. 39, L01104 (2012). doi:10.1029/2011GL049883

E. Dubinin, M. Fraenz, J. Woch et al., Toroidal and poloidal magnetic field at Venus. Venus express observations. Planet. Space Sci. 87, 19-29 (2013a). doi:10.1016/j.pss.2012.12.003

E. Dubinin, M. Fraenz, T.L. Zhang et al., Plasma in the near Venus tail: Venus express observations. J. Geophys. Res. 118, 7624-7634 (2013b). doi:10.1002/2013JA019164

E. Dubinin, M. Fraenz, T.L. Zhang et al., Magnetic fields in the Venus ionosphere: dependence on the IMF direction-Venus express observations. J. Geophys. Res. Space Phys. 119, 7587-7600 (2014). doi:10.1002/2014JA020195

J.P. Eastwood, E.A. Lucek, C. Mazelle et al., The foreshock. Space Sci. Rev. 118, 41-94 (2005). doi:10.1007/ s11214-005-3824-3

J.P. Eastwood, D.A. Brain, J.S. Halekas et al., Evidence for reconnection at Mars. Geophys. Res. Lett. 35, L02106 (2008). doi:10.1029/2007GL032289

N.J.T. Edberg, H. Nilsson, Y. Futaana et al., Atmospheric erosion of Venus during stormy space weather. J. Geophys. Res. 116, A09308 (2011). doi:10.1029/2011JA016749

J.T. Emmert, J.L. Lean, J.M. Picone, Record low thermospheric density during the 2008 solar minimum. Geophys. Res. Lett. 37, L12102 (2010). doi:10.1029/2010GL043671

G. Erdös, A. Balogh, Statistical properties of mirror mode structure observed by Ulysses in the magnetosheath of Jupiter. J. Geophys. Res. 101, 1-12 (1993). doi:10.1029/95JA02207

M.H. Farris, C.T. Russell, Determining the standoff distance of the bow shock: Mach number dependence and use of models. J. Geophys. Res. 99, 17681-17689 (1994). doi:10.1029/94JA01020

A. Fedorov, S. Barabash, J.-A. Sauvaud et al., Measurements of the ion escape rates from Venus for solar minimum. J. Geophys. Res. 116, A07220 (2011). doi:10.1029/2011JA016427

L. Feng, B. Inhester, Y. Wei et al., Morphological evolution of a three-dimensional coronal mass ejection cloud reconstructed from three viewpoints. Astrophys. J. 751, 18 (2012). doi:10.1088/0004-637X/ $751 / 1 / 18$

J.L. Fox, K.Y. Sung, Solar activity variations of the Venus thermosphere/ionosphere. J. Geophys. Res. 106, 21305-21335 (2001). doi:10.1029/2001JA000069

L.A. Frank et al., The plasma instrumentation for the Galileo mission. Space Sci. Rev. 60(1-4), 283-307 (1992). doi:10.1007/BF00216858

Y. Futaana, S. Barabash, M. Yamauchi et al., Mars express and Venus express multi-point observations of geoeffective solar flare events in December 2006. Planet. Space Sci. 56(6), 873-880 (2008). doi:10. 1016/j.pss.2007.10.014

Y. Futaana, J.-Y. Chaufray, H.T. Smith et al., Exospheres and energetic neutral atoms of Mars, Venus and Titan. Space Sci. Rev. 162, 213 (2011). doi:10.1007/s11214-011-9834-4

A. Galli, M.-C. Fok, P. Wurz et al., Tailward flow of energetic neutral atoms observed at Venus. J. Geophys. Res. 113, E00B15 (2008). doi:10.1029/2008JE003096

P. Gary, Electromagnetic ion/ion instabilities and their consequences in space plasmas: a review. Space Sci. Rev. 56, 373-415 (1991). doi:10.1007/BF00196632

J.-C. Gérard, S.W. Bougher, M.A. López-Valverde et al., Aeronomy of the Venus upper atmosphere. Space Sci. Rev. (2017, this issue)

M. Gilmore, A. Treiman, J. Helbert, S. Smrekar, Venus surface composition constrained by observation and experiment. Space Sci. Rev. (2017). doi:10.1007/s11214-017-0370-8

K. Gingauz, I. Klimenko, A. Remizov et al., The VEGA PLASMAG-1 experiment: description and first experimental results. Report KFKI-1986-03/C, Hangarian Acad. Sci., Budapest, Hungary (1986)

E. Golbraikh, M. Gedalin, M. Balikhin et al., Large amplitude nonlinear waves in Venus magnetosheath. J. Geophys. Res. Space Phys. 118, 1706-1710 (2013). doi:10.1002/jgra.50094 
S.W. Good, R.J. Forsyth, Interplanetary coronal mass ejections observed by MESSENGER and Venus express. Sol. Phys. 291(1), 239-263 (2016). doi:10.1007/s11207-015-0828-3

K.I. Gringauz, V.V. Bezrukikh, L.S. Musatov, T.K. Breus, Observation of plasma near Venus on Venera-4 space probe. Kosmich. Issled. 6, 561 (1968)

K.I. Gringauz, V.V. Bezrukikh, T.K. Breus et al., Plasma observations near Venus onboard the Venera 9 and 10 Satellites by means of wide-angle plasma detectors, in Physics of Solar Planetary Environments: Proceedings of the International Symposium on Solar-Terrestrial Physics, vol. II, Boulder, CO, June 7-18, 1976, ed. by D.J. Williams (American Geophysical Union, Washington, 1976). doi:10.1029/SP008p0918

L. Guicking, K.-H. Glassmeier, H.-U. Auster et al., Low-frequency magnetic field fluctuations in Venus' solar wind interaction region: VEX observations. Ann. Geophys. 28, 951-967 (2010). doi:10.5194/angeo-28-951-2010

H. Gunell, E. Kallio, R. Jarvinen et al., Simulations of solar wind charge exchange X-ray emissions at Venus. Geophys. Res. Lett. 34, L03107 (2007). doi:10.1029/2006GL028602

D.A. Gurnett, W.S. Kurth, R.R. Shaw et al., The Galileo plasma wave investigation. Space Sci. Rev. 60, 341-355 (1992). doi:10.1007/978-94-011-2512-3_14

D.A. Gurnett, W.S. Kurth, D.L. Kirchner et al., The Cassini radio and plasma wave investigation. Space Sci. Rev. 114(4), 395-463 (2004). doi:10.1007/s11214-004-1434-0

J.S. Halekas, J.P. Eastwood, D.A. Brain et al., In situ observations of reconnection hall magnetic fields at Mars: evidence for ion diffusion region encounters. J. Geophys. Res. 114, A11204 (2009). doi:10.1029/ 2009JA014544

R.E. Hartle, J.M. Grebowsky, Planetary loss from light ion escape on Venus. Adv. Space Res. 15(4), 117-122 (1995). doi:10.1016/0273-1177(94)00073-A

D.H. Hathaway, The solar cycle. Living Rev. Sol. Phys. 12, 4 (2015). doi:10.1007/lrsp-2015-4

W.R. Hoegy, J.M. Grebowsky, Venus nightside ionospheric holes. J. Geophys. Res. 115, A12322 (2010). doi:10.1029/2010JA015675

D.E. Huddleston, R.J. Strangeway, J. Warnecke et al., Ion cyclotron waves in the Io torus during the Galileo encounter: warm plasma dispersion analysis. Geophys. Res. Lett. 24, 2143-2146 (1997). doi:10.1029/97GL01203

D.M. Hunten, L. Coin, T.M. Donahue, V.I. Moroz (eds.), Venus (Univ. of Arizona Press, Tucson, 1983)

D.S. Intriligator, H.R. Collard, J.D. Mihalov et al., Evidence for Earth magnetospheric tail associated phenomena at $3100 \mathrm{R}_{\mathrm{E}}$. Geophys. Res. Lett. 6(7), 585-588 (1979). doi:10.1029/GL006i007p00585

D.S. Intriligator, J.H. Wolfe, J.D. Mihalov, The Pionerr Venus orbiter plasma analyzer experiment. IEEE Trans. Geosci. Remote Sens. 18(1), 39-43 (1980). doi:10.1109/TGRS.1980.350258

C.M. Jackman, C.T. Russell, D.J. Southwood et al., Strong rapid dipolarization in Saturn's magnetotail: in situ evidence of reconnection. Geophys. Res. Lett. 34, L11203 (2007). doi:10.1029/2007GL029764

B.M. Jakosky, R.P. Lin, J.M. Grebowsky et al., The Mars atmosphere and volatile evolution (maven) mission. Space Sci. Rev. 195(1), 3-48 (2015). doi:10.1007/s11214-015-0139-X

R. Jarvinen, E. Kallio, I. Sillanpää et al., Hybrid modelling the Pioneer Venus orbiter magnetic field observations. Adv. Space Res. 41(9), 1361-1374 (2008). doi:10.1016/j.asr.2007.10.003

R. Jarvinen, E. Kallio, S. Dyadechkin et al., Widely different characteristics of oxygen and hydrogen ion escape from Venus. Geophys. Res. Lett. 37, L16201 (2010). doi:10.1029/2010GL044062

R. Jarvinen, E. Kallio, S. Dyadechkin, Hemispheric asymmetries of the Venusian plasma environment. J. Geophys. Res. 118, 4551-4563 (2013). doi:10.1002/jgra.50387

R. Jarvinen, D.A. Brain, J.G. Luhmann, Dynamics of planetary ions in the induced magnetospheres of Venus and Mars. Planet. Space Sci. 127, 1-14 (2016). doi:10.1016/j.pss.2015.08.012

F.S. Johnson, J.E. Midgley, Notes on the Lunar magnetopshere. J. Geophys. Res. 73, 1523-1532 (1968). doi:10.1029/JA073i005p01523

A.D. Johnstone, K. Glassmeier, M. Acuna et al., Waves in the magnetic field and solar wind flow outside the bow shock at comet Halley. Astron. Astrophys. 187, 47-54 (1987). doi:10.1007/978-3-642-82971-0_7

E. Kallio, R. Jarvinen, P. Janhunen, Venus-solar wind interaction: asymmetries and the escape of ions. Planet. Space Sci. 54(13-14), 1472-1481 (2006). doi:10.1016/j.pss.2006.04.030

E. Kallio, T.L. Zhang, S. Barabash et al., The Venusian induced magnetosphere: a case study of plasma and magnetic field measurements on the Venus express mission. Planet. Space Sci. 56, 796-801 (2008). doi:10.1016/j.pss.2007.09.011

E. Kallio, J-Y. Chaufray, R. Modolo et al., Modeling of Venus, Mars, and Titan. Space Sci. Rev. 162(1), 267-307 (2011). doi:10.1007/s11214-011-9814-8

M.V. Keldysh, Venus exploration with the Venera 9 and 10 spacecraft. Icarus 30, 605-625 (1977). doi:10.1016/0019-1035(77)90085-9

C.F. Kennel, J.P. Edmiston, T. Hada, A quarter century of collisionless shock research, in Collisionless Shocks in the Heliosphere: A Tutorial Review, ed. by R.G. Stone, B.T. Tsurutani. Geophys. Monogr. Ser., vol. 34 (American Geophysical Union, Washington, 1985). doi:10.1029/GM034p0001 
M.G. Kivelson, K.K. Khurana, J.D. Means et al., The Galileo magnetic field investigation. Space Sci. Rev. 60, 357-383 (1992). doi:10.1007/BF00216862

W.C. Knudsen, K.L. Miller, The Venus transterminator ion flux at solar maximum. J. Geophys. Res. 97, 17,165-17,167 (1992). doi:10.1029/92JA01460

W.C. Knudsen, K. Spenner, J. Bakke, V. Novak, Pioneer Venus orbiter planar retarding potential analyzer plasma experiment. IEEE Trans. Geosci. Remote Sens. 18(1), 54-59 (1980). doi:10.1109/TGRS.1980. 350261

W.C. Knudsen, P.M. Banks, K.L. Miller, A new concept of plasma motion and planetary magnetic field for Venus. Geophys. Res. Lett. 9, 765-768 (1982). doi:10.1029/GL009i007p00765

V.A. Krasnopolsky, Lightnings and nitric oxide on Venus. Planet. Space Sci. 31, 1363-1369 (1983). doi:10. 1016/0032-0633(83)90072-7

J.P. Krehbiel, L.H. Brace, R.F. Theis et al., Pioneer Venus orbiter electron temperature probe. IEEE Trans. Geosci. Remote Sens. 18(1), 49-54 (1980). doi:10.1109/TGRS.1980.350260

S.M. Krimgis, D.G. Mitchell, D.C. Hamilton et al., Maghnetospheric imaging instrument (MIMI) on the Cassini mission to Saturn/Titan. Space Sci. Rev. 114, 233-329 (2004). doi:10.1007/s11214-004-1410-8

H. Lammer, J.F. Kasting, E. Chassefière et al., Atmospheric escape and evolution of terrestrial planets and satellites. Space Sci. Rev. 139, 399-436 (2008). doi:10.1007/s11214-008-9413-5

S.A. Ledvina, Y.-J. Ma, E. Kallio et al., Modeling and simulating flowing plasmas and related phenomena. Space Sci. Rev. 139, 143-189 (2008). doi:10.1007/s11214-008-9384-6

K. Liu, E. Kallio, R. Jarvinen et al., Hybrid simulations of the $\mathrm{O}^{+}$ion escape from Venus: influence of the solar wind density and the IMF x component. Adv. Space Res. 43, 1436-1441 (2009). doi:10.1016/ j.asr.2009.01.005

W.A. Livesey, C.F. Kennel, C.T. Russell, ISEE-1 and -2 observations of magnetic field strength overshoots in quasi-perpendicular bow shocks. Geophys. Res. Lett. 9, 1037-1040 (1982). doi:10.1029/ GL009i009p01037

Q. Lu, L. Shan, T. Zhang et al., The role of pickup ions on the structure of the Venusian bow shock and its implications for the termination shock. Astrophys. J. Lett. 773, L24 (2013). doi:10.1088/2041-8205/ 773/2/L24

E.A. Lucek, T.S. Horbury, I. Dondouras et al., Cluster observations of the Earth's quasi-parallel bow shock. J. Geophys. Res. 113, A07S02 (2008). doi:10.1029/2007JA012756

J.G. Luhmann, The solar wind interaction with Venus. Space Sci. Rev. 44, 241-306 (1986). doi:10.1007/ BF00200818

J.G. Luhmann, The solar wind interaction with Venus and Mars: cometary analogies and contrasts, in Cometary Plasma Processes, ed. by A.D. Johnstone. AGU Monograph, vol. 61 (American Geophysical Union, Washington, 1991a), pp. 5-16. doi:10.1029/GM061p0005

J.G. Luhmann, Induced magnetic field at the surface of Venus inferred from Pioneer Venus orbiter nearperiapsis measurements. J. Geophys. Res. 96, 18831-18840 (1991b)

J. Luhmann, Pervasive large-scale magnetic fields in the Venus nightside ionosphere and their implications. J. Geophys. Res. 97, 6103-6121 (1992). doi:10.1029/92JE00514

J.G. Luhmann, The inner magnetosheath of Venus: an analog for Earth? J. Geophys. Res. 100, 12035-12045 (1995). doi:10.1029/94JA02862

J.G. Luhmann, S.J. Bauer, Solar wind effects on atmosphere evolution at Venus and Mars, in Venus and Mars: Atmospheres, Ionospheres, and Solar Wind Interactions, ed. by J.G. Luhmann, M. Tatrallyay, R.O. Pepin. AGU Monograph, vol. 66 (American Geophysical Union, Washington, 1992), pp. 417-430. doi: 10.1029/GM066p0417

J.G. Luhmann, T.E. Cravens, Magnetic fields in the ionosphere of Venus. Space Sci. Rev. 55, 201-274 (1991). doi:10.1007/BF00177138

J.G. Luhmann, J.U. Kozyra, Dayside pickup oxygen ion precipitation at Venus and Mars: spatial distributions, energy deposition and consequences. J. Geophys. Res. 96(A4), 5457-5467 (1991). doi:10.1029/ 90JA01753

J. Luhmann, C.T. Russell, Magnetic field in Venus nightside ionospheric holes: collected Pioneer Venus orbiter magnetometer observations. J. Geophys. Res. 97, 10267-10282 (1992). doi:10.1029/92JE00790

J.G. Luhmann, M. Tatrallyay, C.T. Russell et al., Magnetic fluctuations in the Venus magnetosheath. Geophys. Res. Lett. 10, 655-658 (1983). doi:10.1029/GL010i008p00655

J.G. Luhmann, C.T. Russell, R.C. Elphic, Time scales for the decay of induced large-scale magnetic fields in the Venus ionosphere. J. Geophys. Res. 89, 362-368 (1984). doi:10.1029/JA089iA01p00362

J.G. Luhmann, C.T. Russell, R.C. Elphic, Spatial distribution of magnetic field fluctuations in the dayside magnetosheath. J. Geophys. Res. 91, 1711-1715 (1986). doi:10.1029/JA091iA02p01711

J.G. Luhmann, M. Tatrallyay, R.O. Pepin (eds.), Venus and Mars: Atmospheres, Ionospheres and Solar Wind Interactions. AGU Monograph, vol. 66 (American Geophysical Union, Washington, 1992). doi:10.1029/GM066 
J.G. Luhmann, S.A. Ledvina, J.G. Lyon et al., Venus $\mathrm{O}^{+}$pickup ions: collected PVO results and expectations for Venus express. Planet. Space Sci. 54, 1457-1471 (2006). doi:10.1016/j.pss.2005.10.009

J.G. Luhmann, W.T. Kasprzak, C.T. Russell, Space weather at Venus and its potential consequences for atmosphere evolution. J. Geophys. Res. 112, E04S10 (2007). doi:10.1029/2006JE002820

J.G. Luhmann, Y.J. Ma, M.N. Villarreal et al., The Venus-solar wind interaction: is it purely ionospheric? Planet. Space Sci. 119, 36-42 (2015). doi:10.1016/j.pss.2015.09.012

R. Lundin, Ion acceleration and outflow from Mars and Venus: an overview. Space Sci. Rev. 162, 309-334 (2011). doi:10.1007/s11214-011-9811-y

R. Lundin, A. Zakharov, R. Pellinen et al., Aspera/Phobos measurements of the ion outflow from the MARTIAN ionosphere. Geophys. Res. Lett. 17(6), 873-876 (1990). doi:10.1029/GL017i006p00873

R. Lundin, S. Barabash, Y. Futaana et al., Ion flow and momentum transfer in the Venus plasma environment. Icarus 215, 751-758 (2011). doi:10.1016/j.icarus.2011.06.034

R. Lundin, S. Barabash, Y. Futaana et al., A large-scale flow vortex in the Venus plasma tail and its fluid dynamic interpretation. Geophys. Res. Lett. 40, 1273-1278 (2013). doi:10.1002/grl.50309

Y.J. Ma, A.F. Nagy, C.T. Russell et al., A global multispecies single-fluid MHD study of the plasma around Venus. J. Geophys. Res. Space Phys. 118, 321-330 (2013). doi:10.1029/2012JA018265

G. Mann, H. Lühr, W. Baumjohann, Statistical analysis of short large-amplitude magnetic field structures in the vicinity of the quasi-parallel bow shock. J. Geophys. Res. 99(A7), 13315-13323 (1994). doi:10.1029/94JA00440

C. Martinecz, M. Fränz, J. Woch et al., Location of the bow shock and ion composition boundaries at Venus-initial determinations from VEX ASPERA-4. Planet. Space Sci. 56, 780-784 (2008). doi:10. 1016/j.pss.2007.07.007

C. Martinecz, A. Boesswetter, M. Fränz et al., Plasma environment of Venus: comparison of Venus express ASPERA-4 measurements with 3-D hybrid simulations. J. Geophys. Res. 114, E00B30 (2009). doi: $10.1029 / 2008$ JE003174

K. Marubashi, J. Grebowsky, H. Taylor et al., Magnetic field in the wake of Venus and the formation of ionospheric holes. J. Geophys. Res. 90, 1385-1398 (1985). doi:10.1029/JA090iA02p01385

A. Masters, H.J. McAndrews, J.T. Steinberg et al., Hot flow anomalies at Saturn's bow shock. J. Geophys. Res. 114(A8), A08217 (2009). doi:10.1029/2009JA014112

K. Masunaga, Y. Futaana, M. Yamauchi et al., $\mathrm{O}^{+}$outflow channels around Venus controlled by directions of the interplanetary magnetic field: observations of high energy $\mathrm{O}^{+}$ions around the terminator. J. Geophys. Res. 116, A09326 (2011). doi:10.1029/2011JA016705

K. Masunaga, Y. Futaana, G. Stenberg et al., Dependence of $\mathrm{O}^{+}$escape rate from the Venusian upper atmosphere on IMF directions. Geophys. Res. Lett. 40, 1682-1685 (2013). doi:10.1002/grl.50392

D.L. Matson, L.J. Spilker, J.-P. Lebreton, The Cassini/Huygens mission to the Saturnian system. Space Sci. Rev. 104, 1 (2002). doi:10.1007/978-94-017-3251-2_1

C. Mazelle, F.M. Neubauer, Discrete wave packets at the proton cyclotron at comet P/Halley. Geophys. Res. Lett. 20, 153-156 (1993). doi:10.1029/92GL02613

N. McBride, I. Gilmour (eds.), An Introduction to the Solar System (Cambridge Univ. Press, Cambridge, 2004)

D.J. McComas, H.E. Spence, C.T. Russell et al., The average magnetic field draping and consistent plasma properties of the Venus magnetotail. J. Geophys. Res. 91(A7), 7939-7953 (1986). doi:10. 1029/JA091iA07p07939

D. McComas, R.W. Ebert, H.A. Elliott et al., Weaker solar wind from the polar coronal holes and the whole Sun. Geophys. Res. Lett. 35, L17102 (2008). doi:10.1029/2008GL034896

T.R. McEnulty, J.G. Luhmann, I. dePater et al., Interplanetary coronal mass ejection influence on high energy pick-up ions at Venus. Planet. Space Sci. 58, 1784-1791 (2010). doi:10.1016/j.pss.2010.07.019

K.L. Miller, R.C. Whitten, Ion dynamics in the Venus ionosphere. Space Sci. Rev. 55(1-4), 165-199 (1991). doi:10.1007/BF00177137

U.V. Moestl, N.V. Erkaev, M. Zellinger et al., The Kelvin-Helmholtz instability at Venus: what is the unstable boundary? Icarus 216, 476-484 (2011). doi:10.1016/j.icarus.2011.09.012

C. Möstl, T. Rollett, N. Lugaz et al., Arrival time calculation for interplanetary coronal mass ejections with circular fronts and application to STEREO observations of the 2009 February 13 eruption. Astrophys. J. 741, 1 (2011). doi:10.1088/0004-637X/741/1/34

NASA (ed.), Mariner-Venus 1962, Final project report NASA-SP-59, National Aeronautics and Space Administration, Washington, DC (1965)

M.B. Niedner, J.C. Brandt, Interplanetary gas XXIII. Plasma tail disconnection events in comets: evidence of magnetic field reconnection at interplanetary sector boundaries? Astrophys. J. 223, 655-670 (1978). doi:10.1086/156299

H. Nilsson, N.J.T. Edberg, G. Stenberg et al., Heavy ion escape from Mars, influence from solar wind conditions and crustal magnetic fields. Icarus 215(2), 475-484 (2011). doi:10.1016/j.icarus.2011.08.003 
T. Nordström, G. Stenberg, H. Nilsson et al., Venus ion outflow estimates at solar minimum: influence of reference frames and disturbed solar wind conditions. J. Geophys. Res. Space Phys. 118, 3592-3601 (2013). doi:10.1002/jgra.50305

M. Øieroset, D.L. Mitchell, T.D. Phan et al., Hot diamagnetic cavities upstream of the Martian bow shock. Geophys. Res. Lett. 28, 887-890 (2001a). doi:10.1029/2000GL012289

M. Øieroset, T.D. Phan, M. Fujimoto et al., In situ detection of collisionless reconnection in the Earth's mangetotail. Nature 412, 414-417 (2001b). doi:10.1038/35086520

M. Ong, J.G. Luhmann, C.T. Russell et al., Venus ionospheric "clouds": relationship to the magnetosheath field geometry. J. Geophys. Res. 96, 11,133-11,144 (1991). doi:10.1029/91JA01100

J.L. Phillips, D.L. McComas, The magnetosheath and magnetotail of Venus. Space Sci. Rev. 55, 1-80 (1991). doi:10.1007/BF00177135

S.A. Pope, M.A. Balikhin, T.L. Zhang et al., Giant vortices lead to ion escape from Venus and re-distribution of plasma in the ionosphere. Geophys. Res. Lett. 36, L07202 (2009). doi:10.1029/2008GL036977

K.G. Powell, P.L. Roe, T.J. Linde et al., A solution-adaptive upwind scheme for ideal magnetohydrodynamics. J. Comput. Phys. 154, 284 (1999). doi:10.1006/jcph.1999.6299

R. Ramstad, Y. Futaana, S. Barabash et al., Phobos 2/ASPERA data revisited: planetary ion escape rate from Mars near the 1989 solar maximum. Geophys. Res. Lett. 40, 477-481 (2013). doi:10.1002/grl.50149

W. Reidler, K. Schwingenschuh, Ye.G. Yeroshenko, V.A. Styashkin, C.T. Russell, Magnetic field observations in comet Halley's coma. Nature 321(6067), 288-289 (1986). doi:10.1038/321288a0

Y. Ren, M. Yamada, S. Gerhardt et al., Experimental verification of the hall effect during magnetic reconnection in a laboratory plasma. Phys. Rev. Lett. 95, 055003 (2005). doi:10.1103/PhysRevLett.95.055003

S.A. Romanov, V.N. Smirnov, O.L. Vaisberg, On the nature of the solar wind interaction with Venus. Cosm. Res. 16(5), 746-756 (1978)

Z.J. Rong, S. Barabash, Y. Futaana et al., Morphology of magnetic field in near-Venus magnetotail: VEX observations. J. Geophys. Res. Space Phys. 119, 8838-8847 (2014). doi:10.1002/2014JA020461

A.P. Rouillard, J.A. Davies, R.J. Forsyth et al., A solar storm observed from the Sun to Venus using the STEREO, Venus express, and MESSENGER spacecraft. J. Geophys. Res. 114, A07106 (2009). doi: $10.1029 / 2008$ JA014034

C.T. Russell, Venus aeronomy. Space Sci. Rev. 55, 1-4 (1991). doi:10.1007/978-94-011-3300-5

C.T. Russell, R.C. Elphic, Observation of magnetic flux ropes in the Venus ionosphere. Nature 279(5714), 616-618 (1979). doi:10.1038/279616a0

C.T. Russell, O. Vaisberg, in Venus, ed. by D.M. Hunton et al. (Univ. of Arizona Press, Tucson, 1983), pp. 873-940

C.T. Russell, T.L. Zhang, Unusually distant bow shock encounters at Venus. Geophys. Res. Lett. 19, 833-836 (1992). doi:10.1029/92GL00634

C.T. Russell, R.C. Elphic, J.A. Slavin, Initial Pioneer Venus magnetic field results: dayside observations. Science 203, 745-748 (1979a). doi:10.1126/science.203.4382.745

C.T. Russell, R.C. Elphic, J.A. Slavin, Pioneer magnetometer observations of the Venus bow shock. Nature 282, 815-816 (1979b). doi:10.1038/282815a0

C.T. Russell, R.C. Snare, J.D. Means et al., Pioneer Venus orbiter fluxgate magnetometer. IEEE Trans. Geosci. Remote Sens. 18(1), 32-35 (1980). doi:10.1109/TGRS.1980.350256

C.T. Russell, M.A. Saunders, J.L. Phillips et al., Near-tail reconnection as the cause of cometary tail disconnections. J. Geophys. Res. 91, 1417-1423 (1986). doi:10.1029/JA091iA02p01417

C.T. Russell, E. Chou, J. Luhmann et al., Solar and interplanetary control of the location of the Venus bow shock. J. Geophys. Res. 93, 5461-5469 (1988). doi:10.1029/JA093iA06p05461

C.T. Russell, J.G. Luhmann, K. Schwingenschuhk et al., Upstream waves at Mars: Phobos observations. Geophys. Res. Lett. 17, 897-900 (1990). doi:10.1029/GL017i006p00897

C.T. Russell, K.K. Khurana, D.E. Huddleston et al., Localized reconnection in the near Jovian magnetotail. Science 280, 1061-1064 (1998). doi:10.1126/science.280.5366.1061

C.T. Russell, S.S. Mayerberger, X. Blanco-Cano, Proton-cyclotron waves at Mars and Venus. Adv. Space Res. 38, 745-751 (2006a). doi:10.1016/j.asr.2005.02.091

C.T. Russell, R.J. Strangeway, T.L. Zhang, Lightning detection on the VEX mission. Planet. Space Sci. 54, 1344-1351 (2006b). doi:10.1016/j.pss.2006.04.026

C.T. Russell, T.L. Zhang, M. Delva et al., Lightning on Venus inferred from whistler-modewaves in the ionosphere. Nature 450, 661-662 (2007). doi:10.1038/nature05930

C.T. Russell, T.L. Zhang, R.J. Strangway et al., Electromagnetic waves observed by VEX at periapsis: detection and analysis techniques. Adv. Space Res. 41, 113-117 (2008a). doi:10.1016/j.asr.2007.08.032

C.T. Russell, T.L. Zhang, H.Y. Wei, Whistler mode waves from lightning on Venus: magnetic control of ionospheric access. J. Geophys. Res. 113, E00B05 (2008b). doi:10.1029/2008JE003137

C.T. Russell, H. Leinweber, T.L. Zhang et al., Electromagnetic waves observed on a flight over a Venus electrical storm. Geophys. Res. Lett. 40, 216-220 (2013a). doi:10.1029/2012GL054308 
C.T. Russell, H. Leinweber, R.A. Hart et al., Venus express observations of ULF and ELF waves in the Venus ionosphere: wave properties and sources. Icarus 226, 1527-1537 (2013b). doi:10.1016/j.icarus.2013. 08.019

C.T. Russell, J.G. Luhmann, R.J. Strangeway, Space Physics: An Introduction (Cambridge Univ. Press, Cambridge, 2016)

M.A. Saunders, C.T. Russell, Average dimension and magnetic structure of the distance Venus magnetotail. J. Geophys. Res. 91, 5589-5604 (1986). doi:10.1029/JA091iA05p05589

R. Saxena, S.D. Bale, T.S. Horbury, Wavelength and decay length of density overshoot structure in supercritical, collisionless bow shocks. Phys. Plasmas 12, 052904 (2005). doi:10.1063/1.1900093

F.L. Scarf, W.W.L. Taylor, P.F. Virobik, Pioneer Venus orbiter plasma wave investigation. IEEE Trans. Geosci. Remote Sens. 18(1), 36-38 (1980). doi:10.1029/JA085iA13p07599

G. Schubert, K. Schwartz, A theory for the interpretation of Lunar surface magnetometer data. Moon 1, 106 (1969). doi:10.1007/BF00561773

S.J. Schwartz, C.P. Chaloner, P.J. Christiansen et al., An active current sheet in the solar wind. Nature 318, 269-271 (1985). doi:10.1038/318269a0

S.J. Schwartz, D. Burgess, W.P. Wilkinson et al., Observations of short large-amplitude magnetic structures at a quasi-parallel shock. J. Geophys. Res. 97, 4209-4227 (1992). doi:10.1029/91JA02581

S.J. Schwartz, G. Paschmann, N. Sckopke et al., Conditions for the formation of hot flow anomalies at Earth's bow shock. J. Geophys. Res. 105, 12639-12650 (2000). doi:10.1029/1999JA000320

L. Shan, Q. Lu, M. Wu et al., Transmission of large-amplitude ULF waves through a quasi-parallel shock at Venus. J. Geophys. Res. 119, 237-245 (2014). doi:10.1002/2013JA019396

L. Shan, Q. Lu, C. Mazelle et al., The shape of the Venusian bow shock at solar minimum and maximum: revisit based on VEX observations. Planet. Space Sci. 109-110, 32-37 (2015). doi:10.1016/j.pss.2015. 01.004

V.I. Shematovich, D.V. Bisikalo, S. Barabash et al., Monte Carlo study of interaction between solar wind plasma and Venusian upper atmosphere. Sol. Syst. Res. 48(5), 317-323 (2014). doi:10.1134/ S0038094614050049

K. Shibata, New observational facts about solar flares from Yohkoh studies-evidence of magnetic reconnection and a unified model of flares. Adv. Space Res. 17, 9-18 (1996). doi:10.1016/02731177(95)00534-L

D.G. Sibeck, N.L. Borodkova, G.N. Zastenker et al., Gross deformation of the dayside magnetosphere. Geophys. Res. Lett. 25, 453-456 (1998). doi:10.1029/98GL00134

D.G. Sibeck, N.L. Borodkova, S.J. Schwartz, Comprehensive study of the magnetospheric response to a hot flow anomaly. J. Geophys. Res. 104, 4577-4593 (1999). doi:10.1029/1998JA900021

SILSO, World data center-sunspot number and long-term solar observations. On-line sunspot number catalogue. Royal Observatory of Belgium, http://www.sidc.be/SILSO/ (1960-2015)

J.A. Slavin, M.H. Acuña, B.J. Anderson et al., MESSENGER and Venus express observations of the solar wind interaction with Venus. Geophys. Res. Lett. 36, L09106 (2009). doi:10.1029/2009GL037876

S.C. Solomon, R.L. McNutt Jr., R.E. Gold et al., The MESSENGER mission to Mercury: scientific objectives and implementation. Planet. Space Sci. 49, 1445-1465 (2001). doi:10.1016/S0032-0633(01)00085-X

A.J. Somogyi, K.I. Gringauz, K. Szego et al., First observations of energetic particles near comet Halley. Nature 321(6067), 285-288 (1986). doi:10.1038/321285a0

C.P. Sonett, D.S. Colburn, Establishment of a Lunar unipolar generator and associated shock and wake by the solar wind. Nature 216, 340-343 (1967). doi:10.1038/216340a0

P. Song, C.T. Russell, What do we really know about the magnetosheath? Adv. Space Res. 20, 747-765 (1997). doi:10.1016/S0273-1177(97)00466-3

R.J. Strangeway, C.T. Russell, Plasma waves and field-aligned currents in the Venus plasma mantle. J. Geophys. Res. 101, 17,313-17,324 (1996). doi:10.1029/96JA00927

T. Tanaka, Configurations of the solar wind flow and magnetic field around the planets with no magnetic field: calculation by a new MHD simulation scheme. J. Geophys. Res. 98(A10), 17,251-17,262 (1993). doi:10.1029/93JA01516

T. Tanaka, Effects of decreasing ionospheric pressure on the solar wind interaction with non-magnetized planets. Earth Planets Space 50(3), 259-268 (1998). doi:10.1186/BF03352112

T. Tanaka, K. Murawski, Three-dimensional MHD simulation of the solar wind interaction with the ionosphere of Venus: results of a two-component reacting plasma simluation. J. Geophys. Res. 102(A9), 19,805-19,821 (1997). doi:10.1029/97JA01474

W.W.L. Taylor, F.L. Scarf, C.T. Russell et al., Absorption of whistler mode waves in the ionosphere if Venus. Science 205, 112-114 (1979). doi:10.1126/science.205.4401.112

H.A. Taylor, H.C. Brinton, T.C.G. Wagner, B.H. Blackwell, G.R. Cordier, Benett ion mass spectrometers on the Pioneer Venus bus and orbiter. IEEE Trans. Geosci. Remote Sens. 18(1), 44-49 (1980). doi:10.1109/TGRS.1980.350259 
N. Terada, S. Mashida, H. Shinagawa, Global hybrid simulation of the Kelvin-Helmholtz instability at the Venus ionopause. J. Geophys. Res. 107(A12), 1471 (2002). doi:10.1029/2001JA009224

N. Terada, H. Shinagawa, T. Tanaka et al., A three-dimensional, multispecies, comprehensive MHD model of the solar wind interaction with the planet Venus. J. Geophys. Res. 114, A09208 (2009). doi:10.1029/2008JA013937

M.F. Thomsen, J.T. Gosling, S.A. Fuselier et al., Hot, diamagnetic cavities upstream from the Earth's bow shock. J. Geophys. Res. 91, 2961-2973 (1986). doi:10.1029/JA091iA03p02961

S.M.E. Tsang, A.J. Coates, G.H. Jones et al., Ionospheric photoelectrons at Venus: case studies and first observation in the tail. Planet. Space Sci. 113-114, 385-394 (2015). doi:10.1016/j.pss.2015.01.019

B.T. Tsurutani, E.J. Smith, R.R. Anderson et al., Lion roars and nonoscillatory drift mode mirror waves in the magnetosheath. J. Geophys. Res. 87, 6060-6072 (1982). doi:10.1029/JA087iA08p06060

B.T. Tsurutani, E.J. Smith, H. Matsumoto et al., Highly nonlinear magnetic pulses at comet Giacobini-Zinner. Geophys. Res. Lett. 17, 757-760 (1990). doi:10.1029/GL017i006p00757

B.T. Tsurutani, J.K. Arballo, E.J. Smith et al., Large-amplitude magnetic pulses downstream of the Jovian bow shock: Ulysses observations. Planet. Space Sci. 41, 851-856 (1993). doi:10.1016/00320633(93)90092-G

J.M. Turner, L.F. Burlaga, N.F. Ness et al., Magnetic holes in the solar wind. J. Geophys. Res. 82, 1921-1924 (1977). doi:10.1029/JA082i013p01921

O.L. Vaisberg, L.M. Zeleny, Formation of the plasma mantle in the Venusian magnetosphere. Icarus 58, 412-430 (1984). doi:10.1016/0019-1035(84)90087-3

O.L. Vaisberg, A.V. Bogdanov, N.V. Borodin et al., Possible crossing of geomagnetic wake at the distance of 3000 Earth's radii during the flight of interplanetary automatic station Mars-3. Dokl. USSR Acad. Sci. 203(2), 309-310 (1972)

O.L. Vaisberg, S.A. Romanov, V.N. Smirnov et al., Ion flux parameters in the solar wind-Venus interaction region, in Physics of Solar Planetary Environment, ed. by D.J. Williams (AGU, Boulder, 1976), pp. 904917. doi:10.1029/SP008p0904

O. Vaisberg, A. Fedorov, F. Dunjushkin, A. Kozhukhovsky, V. Smirnov, L. Avanov, C.T. Russell, J.G. Luhmann, Ion Populations in the tail of Venus. Adv. Space Res. 16(4), 105-118 (1995). doi:10.1016/ 0273-1177(95)00217-3

I.Y. Vasko, L.M. Zelenyi, A.V. Artemyev et al., The structure of the Venusian current sheet. Planet. Space Sci. 96, 81-89 (2014). doi:10.1016/j.pss.2014.03.013

D. Vech, K. Szego, A. Opitz et al., Space weather effects on the bow shock, the magnetic barrier and the ion composition boundary at Venus. J. Geophys. Res. 120, 4613-4627 (2015). doi:10.1002/2014JA020782

D. Vech, G. Stenberg, H. Nilsson et al., Statistical features of the global polarity reversal of the Venusianinduced magnetosphere in response to the polarity change in interplanetary magnetic field. J. Geophys. Res. Space Phys. 121, 3951-3962 (2016). doi:10.1002/2015JA021995

M.I. Verigin, K.I. Gringauz, T. Gombosi et al., Plasma near Venus from the Venera 9 and 10 wide-angle analyzer data. J. Geophys. Res. 83(A8), 3721-3728 (1978). doi:10.1029/JA083iA08p03721

M.I. Verigin, J. Slavin, A. Szabo et al., Unusually distant bow shock encounters at Mars: analysis of March 24, 1989 event. Space Sci. Rev. 111, 233-243 (2004). doi:10.1007/978-0-306-48604-3_7

M.N. Villarreal, C.T. Russell, H.Y. Wei et al., Characterizing the low-altitude magnetic belt at Venus: complementary observations from the Pioneer Venus orbiter and Venus express. J. Geophys. Res. Space Phys. 120, 2232-2240 (2015). doi:10.1002/2014JA020853

M. Volwerk, M.G. Kivelson, K.K. Khurana, Wave activity in Europa's wake: implications for ion pickup. J. Geophys. Res. 106, 26033-26048 (2001). doi:10.1029/2000JA000347

M. Volwerk, T.L. Zhang, M. Delva et al., First identification of mirror mode waves in Venus' magnetosheath. Geophys. Res. Lett. 35, L12204 (2008a). doi:10.1029/2008GL033621

M. Volwerk, T.L. Zhang, M. Delva et al., Mirror-mode-like structures in Venus' induced magnetosphere. J. Geophys. Res. 113, E00B16 (2008b). doi:10.1029/2008JE003154

M. Volwerk, M. Delva, Y. Futaana, A. Retino, Z. Voros, T.L. Zhang, W. Baumjohann, S. Barabash, Substorm activity in Venus's magnetotail. Ann. Geophys. 27, 2321-2330 (2009). doi:10.5194/angeo-272321-2009

Z. Vörös, T.L. Zhang, M.P. Leuber et al., Magnetic fluctuations and turbulence in the Venus magnetosheath and wave. Geophys. Res. Lett. 35, L11135 (2008a). doi:10.1029/2008GL033879

Z. Vörös, T.L. Zhang, M.P. Leuber et al., Intermittent turbulence, noisy fluctuations, and wavy structures in the Venusian magnetosheath and wake. J. Geophys. Res. 113, E00B21 (2008b). doi:10.1029/ 2008JE003159

J.H. Waite, W.S. Lewis, W.T. Kasprzak et al., The Cassini ion and neutral mass spectrometer (INMS) investigation. Space Sci. Rev. 114(1), 113-231 (2004). doi:10.1007/s11214-004-1408-2

R. Walker, T. Terasawa, S.P. Christon et al., Source and loss processes in the magnetotail. Space Sci. Rev. 88(1-2), 285-353 (1999). doi:10.1023/A:1005207918263 
S.N. Walker, M.A. Balikhin, T.L. Zhang et al., Unusual nonlinear waves in the Venusian magnetosheath. J. Geophys. Res. 116, A01215 (2011). doi:10.1029/2010JA015916

H.Y. Wei, C.T. Russell, T.L. Zhang et al., Comparison study of magnetic flux ropes in the ionospheres of Venus, Mars and Titan. Icarus 206, 174-181 (2010). doi:10.1016/j.icarus.2009.03.014

H.Y. Wei, C.T. Russell, T.L. Zhang et al., Comparative study of ion cyclotron waves at Mars, Venus, and Earth. Planet. Space Sci. 59, 1039-1047 (2011). doi:10.1016/j.pss.2010.01.004

H.Y. Wei, C.T. Russell, J.T.M. Daniels et al., Observations of quasi-perpendicular propagating electromagnetic waves near the ionopause current sheet of Venus. J. Geophys. Res. 117, A05330 (2012a). doi:10.1029/2011JA017374

Y. Wei, M. Fraenz, E. Dubinin et al., A teardrop-shaped ionosphere at Venus in tenuous solar wind. Planet. Space Sci. 73(1), 254-261 (2012b). doi:10.1016/j.pss.2012.08.024

I. Whittaker, G. Guymer, M. Grande et al., Venusian bow shock as seen by the ASPERA-4 ion instrument on VEX. J. Geophys. Res. 115, A09224 (2010). doi:10.1029/2009JA014826

D.J. Williams, R.W. McEntire, S. Jaskulek, B. Wilken, The Galileo energetic particles detector. Space Sci. Rev. 60, 385-412 (1992). 1992

A.O. Williams, N.J.T. Edberg, S.E. Milan et al., Tracking corotating interaction regions from the Sun through to the orbit of Mars using ACE, MEX, VEX, and STEREO. J. Geophys. Res. 116, A08103 (2011). doi:10.1029/2010JA015719

M. Yamauchi, Y. Futaana, A. Fedorov et al., Comparison of accelerated ion populations observed upstream of the bow shocks at Venus and Mars. Ann. Geophys. 29, 511-528 (2011). doi:10.5194/ angeo-29-511-2011

Ye.G. Yeroshenko, Unipolar induction effects in the magnetic tail of Venus. Cosm. Res. 20, 604 (1979)

D.T. Young, J.J. Berthelier, M. Blanc et al., Cassini plasma spectrometer investigation. Space Sci. Rev. 114(1), 1-112 (2004). doi:10.1007/s11214-004-1406-4

L.M. Zelenyi, H.V. Malova, A.V. Artemyev et al., Thin current sheets in collisionless plasma: equilibrium structure, plasma instabilities, and particle acceleration. Plasma Phys. Rep. 37, 118-160 (2011). doi:10.1134/S1063780X1102005X

T.L. Zhang, M. Delva, W. Baumjohann et al., Little or no solar wind enters Venus' atmosphere at solar minimum. Nature 450, 654-656 (2007a). doi:10.1038/nature06026

T.L. Zhang, G. BergHofer, W. Magnes et al., MAG: the fluxgate magnetometer for Venus express. ESA SP 1295, 1-10 (2007b)

T.L. Zhang, M. Delva, W. Baumjohann et al., Initial Venus express magnetic field observations of the Venus bow shock location at solar minimum. Planet. Space Sci. 56, 785-789 (2008a). doi:10.1016/ j.pss.2007.09.012

T.L. Zhang, M. Delva, W. Baumjohann et al., Initial Venus express magnetic field observations of the magnetic barrier at solar minimum. Planet. Space Sci. 56, 790-795 (2008b). doi:10.1016/j.pss.2007.10.013

T.L. Zhang, S. Pope, M. Balikhin et al., Venus express observations of an atypically distant bow shock during the passage of an interplanetary coronal mass ejection. J. Geophys. Res. 113, E00B12 (2008c). doi:10.1029/2008JE003128

T.L. Zhang, C.T. Russell, W. Zambelli et al., Behaviour of current sheets at directional magnetic discontinuities in the solar wind at 0.72 AU. Geophys. Res. Lett. 35, L24102 (2008d). doi:10.1029/2008GL036120

T.L. Zhang, C.T. Russell, W. Baumjohann et al., Characteristic size and shape of the mirror mode structures in the solar wind at 0.72 AU. Geophys. Res. Lett. 35, L10106 (2008e). doi:10.1029/2008GL033793

T.L. Zhang, J. Du, Y.J. Ma et al., Disappearing induced magnetosphere at Venus: implications for close-in exoplanets. Geophys. Res. Lett. 36, L20203 (2009). doi:10.1029/2009GL040515

T.L. Zhang, W. Baumjohann, J. Du et al., Hemispheric asymmetry of the magnetic field wrapping pattern in the Venusian tail. Geophys. Res. Lett. 37, L14202 (2010). doi:10.1029/2010GL044020

T.L. Zhang, Q.M. Lu, W. Baumjohann et al., Magnetic reconnection in the near Venusian magnetotail. Science 336, 567-570 (2012). doi:10.1126/science.1217013

T.L. Zhang, W. Baumjohann, C.T. Russell et al., A statistical study of the low-altitude ionospheric magnetic fields over the north pole of Venus. J. Geophys. Res. Space Phys. 120, 6218-6229 (2015). doi:10.1002/ 2015JA021153 